Foundation of Physics manuscript No.

(will be inserted by the editor)

\title{
The Milky Way's Supermassive Black Hole: How good a case is it?
}

\author{
A Challenge for Astrophysics $\&$ Philosophy of Science
}

\author{
Andreas Eckart ${ }^{a, b}$ Andreas Hüttemann $^{c}$, \\ Claus Kiefer ${ }^{d}$, Silke Britzen ${ }^{b}$, Michal \\ Zajaček $^{a, b}$, Claus Lämmerzahl ${ }^{e}$, Manfred \\ Stöckler $^{f}$, Monika Valencia-S. ${ }^{a}$, Vladimir \\ Karas $^{g}$, Macarena García-Marín ${ }^{h}$
}

Received: date / Accepted: date

\begin{abstract}
The compact and, with $4.3 \pm 0.3 \times 10^{6} \mathrm{M}_{\odot}$, very massive object located at the center of the Milky Way is currently the very best candidate for a supermassive black hole $(\mathrm{SMBH})$ in our immediate vicinity. The strongest evidence for this is provided by measurements of stellar orbits, variable X-ray emission, and strongly variable polarized near-infrared emission from the location of the radio source Sagittarius A* (SgrA*) in the middle of the central stellar cluster. Simultaneous near-infrared and X-ray observations of SgrA* have revealed insights into the emission mechanisms responsible for the powerful near-infrared and X-ray flares from within a few tens to one hundred Schwarzschild radii of such a putative SMBH at the center of the Milky Way. If $\mathrm{SgrA}^{*}$ is indeed a $\mathrm{SMBH}$ it will, in projection onto the sky, have the largest event horizon and will certainly be the first and most important target of the Event Horizon Telescope (EHT) Very Long Baseline Interferometry (VLBI) observations currently being prepared. These observations in combination with the infrared interferometry experiment GRAVITY at the Very Large Telescope Interferometer (VLTI) and other experiments across the electromagnetic spectrum might yield proof for the presence of a black hole at the center of the Milky Way. The large body of evidence continues to discriminate the identification of $\mathrm{SgrA}^{*}$ as a SMBH from alternative possibilities. It is, however, unclear when the ever mounting evidence for $\mathrm{SgrA}^{*}$ being associated with a SMBH will suffice as a convincing proof. Additional compelling evidence may come from future gravitational wave observatories. This manuscript reviews the observational facts, theoretical grounds and conceptual aspects for the case of SgrA* being a black hole. We treat theory and observations in the framework of the philosophical discussions about "(Anti)Realism and Underdetermination", as this line of arguments allows us to describe the situation in observational astrophysics with respect to supermassive black holes. Questions concerning the existence of supermassive black holes and in particular $\mathrm{SgrA}^{*}$ are discussed using causation as an indispensable element.
\end{abstract}

Corresponding author: Andreas Eckart; eckart@ph1.uni-koeln.de

a) I. Physikalisches Institut, Universität zu Köln, Zülpicher Straße 77, 50937 Köln, Germany

b) Max-Planck-Institut für Radioastronomie, Auf dem Hügel 69, 53121 Bonn, Germany

c) Philosphisches Seminar, Albertus-Magnus-Platz 1, 50923 Köln, Germany

d) Institut für Theoretische Physik, Zülpicher Straße 77, 50937 Köln, Germany

e) Center for Applied Space Technology and Microgravity (ZARM), Am Fallturm, 28359 Bremen, Germany

f) Universität Bremen, Institut für Philosophie, FB 9, Postfach 330 440, Enrique-Schmidt-Str. 7, 28359 Bremen, Germany

g) Astronomical Institute, Academy of Sciences, Boční II 1401, CZ-14131 Prague, Czech Republic

h) European Space Agency (ESA/STScI), 3700 San Martin Drive, Baltimore, MD 21218, USA 
We show that the results of our investigation are convincingly mapped out by this combination of concepts.

Keywords Black Holes: mass, spin, charge - Sources: Sagittarus A* Galaxies: Active · Philosophy of Science: (Anti)Realism, Underdetermination, Causality

\section{Introduction}

The compact radio source Sagittarius $\mathrm{A}^{*}\left(\mathrm{SgrA}^{*}\right)$ is located within a dense central stellar cluster surrounded by a small ( $\sim 1$ arcsecond in angular and 0.04 parsec linear diameter) cluster of high velocity stars (Reid et al, 2003b; Ghez et al, 2008; Gillessen et al, 2009a). SgrA* is active across the entire electromagnetic spectrum and subject to detailed investigations (e.g. Genzel et al, 2010; Ghez et al, 2009; Eckart et al, 2005). In this contribution, we summarize some of the most intriguing characteristics of the central dark mass of $4.3 \pm 0.3 \times 10^{6} \mathrm{M} \odot 1$ associated with $\operatorname{Sgr} \mathrm{A}^{*}$ and the stars in its vicinity as well as the conceptual aspects - including a summary of its philosophical background - that may lead to a convincing proof for the existence of a supermassive black hole (SMBH). Our knowledge on the Galactic Center (GC) profits greatly from the very recent results obtained with large 8-10 $\mathrm{m}$ class ground based telescopes that operate in the infrared? Only at these wavelengths it is possible to observe the high velocity stars and to monitor the optically thin synchrotron radiation from its immediate vicinity. At infrared wavelengths, the dust and gas along the $\sim 8 \mathrm{kpc}$ line of sight towards the center can be penetrated, resulting in detailed data on the stars and the infrared counterpart of SgrA*. Additional observational data stem from highest resolution radio-interferometric observations in the $\mathrm{mm} / \mathrm{sub}-\mathrm{mm}$ bands. A new and for this purpose specialized array of mm-antennas - the Event-Horizon-Telescope (EHT $\left.{ }^{3}\right)$ - has already performed first observations of Sgr $\mathrm{A}^{*}$ at $1 \mathrm{~mm}$ and supplementary Very Long Baseline Interferometry (VLBI) information at $3 \mathrm{~mm}$ and longer wavelengths is available. Strong variability from the radio through the infrared to the X-ray domain also indicates that energetic processes like accretion onto a black hole may be dominating the center of the Milky Way.

Although the observational evidence for SgrA* being a SMBH is ever increasing, one has to ask the question: At which point do we have enough evidence to claim that $S_{g r A}$ * is a $S M B H$ ? This requires consensus on testable predictions based on a definition of a SMBH as well as on the epistemological processes that need to be followed. Therefore, the paper has two focal points: One on the philosophy of science and the other on an analysis of current theoretical and observational facts that need to be considered within the philosophical framework.

SMBHs are philosophically interesting entities given that they are only observable by indirect means. Within the philosophy of science, the question on the reality of such unobservable entities, historically mostly part of the microcosm, has a long tradition (see e.g. Chakravartty, 2010, for a review and the Appendix $\mathrm{A}$ for an example). We note that Ian Hacking has stressed a special status for astrophysics in the discussion of scientific realism (Hacking, 1989). As a logical consequence of his experimental criteria for realism (see Hacking, 1983; Sandell, 2010) that require the possibility of interaction with and manipulation of the entity in question, he

1 In the context of the Galactic Center, the black hole mass is given in millions of solar masses $\left(\mathrm{M}_{\odot}=1.98 \times 10^{30} \mathrm{~kg}\right)$.

2 e.g. the European Southern Observatory's Very Large Telescope on Paranal in Chile or the W.M. Keck Observatory's Keck telescopes on Mauna Kea in Hawai'i, USA

3 The EHT is a Very Long Baseline Interferometry (VLBI) array working at millimeter wavelengths, and dedicated for observing the event horizons (but see section 2.2) of the largest SMBHs in the sky; http://www.eventhorizontelescope.org/ 
is committed to astrophysical anti-realism due to the observational nature of astrophysical research. In particular, he uses the existence of not directly observable gravitational microlenses in favor of his argument. From the existence of gravitational microlenses he deduces an inherent underdetermination of astrophysical theories, that makes their results apparently less reliable in comparison with experimental disciplines that allow for a direct interaction with the entities under investigation.

For the current paper it needs to be clarified if supermassive black holes are different entities compared to, for example electrons, chairs, or galaxies, such that a special consideration in the framework of philosophy of science is justified. While all of them can be discriminated from each other in the framework of an constructive empiricism, there are noteworthy differences: Entities like chairs or tables stand out with respect to the imperfection of their definition (as outlined by, for example, David Hume; see Nelson, 2010), galaxies stand out with respect to their distance and complexity (these object have been considered by van Fraassen, 1985), and electrons are very simple entities that can be "sprayed" (Hacking, 1989), this means, they are readily available in the laboratory for causality test. Black holes, and in particular supermassive black holes stand out in the simplicity of their mathematical definition that is best expressed in the "no-hair" theorem (see section 2.1). They are not readily available in the laboratory and cannot easily be "sprayed". They are characterized by an event horizon that, however, cannot become part of an external observer's past in a finite time but is an important discriminator against other similarly compact and massive objects.

Claiming that black holes are only an artifact of classical theory and thereby compromising on the seriousness with which we treat the event horizon makes the problem even worse. In this case, it is even more difficult to distinguish between the black hole solution and other descriptions of compact objects of the same mass.

Black holes may, hence, be seen as the prototype of astrophysical entities that suffer from underdetermination. There is, however, ample indirect evidence for their existence. Still, as motivated by Hacking's line of arguments, it may be interesting to raise the epistemological question of whether we face a situation of underdetermination in the search for SMBHs. How strong is the available evidence in favor of the SMBH interpretation, being one possible "causal story" to unify the "existing evidentiary traces" (e.g. Cleland, 2002; Anderl, 2015, 2016; Smeenk, 2005). Are there alternative theories that may as well serve as possible explanations for our observations? If yes, what kind of observations would we need to disprove alternative theories or decide in favor of one of them? How can we describe and organize the epistemological situation we face in a formal way?

In order to investigate these questions, we first summarize the theoretical predictions and initial observational evidences for black holes and, in particular, the SMBH at the center of the Milky Way in section 2. This includes the history of the evolution of the subject and considerations. We then elaborate on the philosophical questions connected to the identification of SMBHs in section 3. In section 4 we present a detailed description and evaluation of mostly observationally based results that point at the presence of a SMBH at the Galactic Center. We then summarize possible alternatives to SMBHs in section 5 and mention their possible astrophysical consequences. In section [6] we summarize some of the important future possibilities that arise with new instrumentation. A synthesis of all facts is attempted in section 7 In section 8, we give a summary and conclusions in the framework of the philosophy of science principles outlined earlier. 


\section{The concept of Black Holes}

Objects that can be so compact that light cannot escape their gravitational fields were first speculated on in the 18th century by John Michell and Pierre-Simon Laplace 4 . In 1916, Karl Schwarzschild (Schwarzschild, 1916) found a solution of Einstein's field equations that can describe a non-rotating black hole in vacuum, although not called like this at that time. In the same year, Hans Reissner (Reissner, 1916) found the solution for a stationary charged point mass, which was extended by Gunnar Nordström (Nordström, 1918) for a spherically symmetric charged body. In 1939, Einstein speculated that ultra-compact masses do not exist (Einstein, 1939). In 1939/40, the authors of Oppenheimer and Volkoff (1939) and Oppenheimer and Snyder (1939) worked on continued gravitational contraction and resulting objects. It was David Finkelstein in 1958 (Finkelstein, 1958) who highlighted that this was a region of space from which virtually no information could escape. These objects were referred to as "frozen stars" at that time and early relativists often used the term Schwarzschild's sphere, discontinuity, sphére catastrophique, magic circle or just frontier or barrier (Bartusiak, 2015). Soon after that it could be shown that black holes actually can generically be predicted from general relativity (GR). Between the late 1940's and 1960's, Ann Ewing published articles on astronomy and physics in Science News (Brown, 2010). She used the term "black hole" as early as 1964 in her article "Black Holes in Space" (Ewing, 1964) following a contribution at a meeting of the American Association for the Advancement of Science (AAAS) (Brown, 2010). The most recent investigation on the introduction of the term "black hole" in astrophysics is given by Bartusiak (2015) and summarized here briefly: She explains that, as reported by the editor of the Life Magazine Albert Rosenfeld, the astrophysicists Fred Hoyle and William Fauler used the term at the 1963 Texas Symposium. It was used again at the AAAS meeting a short time later by HongYee Chiu from the Goddard institute. Hong-Yee Chiu reports that the term may have been used around 1960 by the Princeton physicist Robert Dicke. Ann Ewing had attended that very AAAS meeting in 1963. This was quickly followed by the discovery of stellar black hole candidates. Bartusiak (2015) reports that John Wheeler may have been inspired by a poem titled "Music of the Spheres" by A.M. Sullivan (1896-1980). The poem focuses on William Herschel and uses the term "black hole"15. It was published in the New York Times in August, 1967. In that very year John Wheeler proposed to use the term "black hole" as a technical term; from that time on it became the standard term.

The theoretical structure of stationary black holes as solutions to Einstein's field equations was clarified in the 1960s. Stationary black holes can be formed as the asymptotic end state of a gravitational collapse. A most interesting result is the validity of uniqueness theorems for black holes (see e.g. Heusler, 1996). Within the Einstein-Maxwell theory (describing coupled gravitational and electromagnetic fields) one can prove that black holes are uniquely characterized by only three parameters: mass $M$, angular momentum $J \equiv c M a$ (see footnote 6 and details given below and in section 4.4), and electric charge $q$. In a sense, black holes are thus much simpler objects than ordinary stars - stationary black holes with the same three parameters look the same. "Dark stars" that do not collapse can be shown to be unstable (Cardoso et al, 2014). During the gravitational collapse of a star to a black hole, all other degrees of freedom are radiated away, mostly in the form of gravitational waves ("ringdown"). Since one can refer to these other

\footnotetext{
4 For a detailed account for the history of black holes, see Israel (1987).

5 "When the long eye of Herschel Burrowed the heavens Near the belt of Orion He trembled in awe At the black hole of Chaos".

6 For black holes the spin can be characterized by the angular momentum parameter $a=J / M c$, where $J$ is the angular momentum, $M$ the mass of the black hole, and $c$ is the speed of light
} 
degrees of freedom as "hair", the uniqueness theorems are also known under the name no-hair theorems.

The most general solution, with $M, a$, and $q$ non-vanishing, is called KerrNewman solution. Since $q$ is thought to be irrelevant in astrophysical situations, the most important case for astrophysics is the Kerr solution, found by Roy Kerr in 1963 (Kerr, 1963); it is fully characterized by $M$ and $a$. To the extent that the contribution of surrounding matter is negligible, the black-hole candidates discussed in our paper are all expected to be described by this solution. A black-hole solution with $a=0$ is spherically symmetric and called Schwarzschild solution $(M \neq 0$, $q=0)$ or Reissner-Nordström solution $(M \neq 0, q \neq 0)$ (see also comments in section 4.12).

The concrete values of the few black hole parameters $M, a$, and $q$ must be obtained through observations of the black hole's interaction with its immediate environment. This is mostly accomplished by observing the strong electromagnetic radiation produced during these interactions. The high luminosity of extragalactic sources found first at optical and then at radio wavelengths drew the attention to possible high mass concentrations in the nuclei of galaxies. SMBHs were first introduced in order to explain the large luminosities of quasi-stellar objects (QSOs) and their radio counterparts called quasars. These objects have been found to outshine their host galaxies at the centers of which they are located.

QSOs and quasars are believed to be powered by accretion of material into SMBHs (Zel'dovich and Novikov, 1964; Salpeter, 1964; Rees, 1984). From first principles, it can be shown that this process very efficiently allows us to generate the required high luminosities. Relativistic effects like superluminal motion (apparent motion faster than the speed of light) in jets, black hole mass measurements and high X-ray and $\gamma$-ray luminosities are further strong evidences for the presence of supermassive compact objects like SMBHs at the centers of galaxies.

In 1971, Donald Lynden-Bell and Martin Rees speculated on the presence of a SMBH at the center of the Milky Way. Three years later, the compact and stationary synchrotron radio source SgrA* was discovered by Balick and Brown (1974) using radio interferometry. Hence on theoretical and observational grounds, a SMBH at the center of the Milky Way had to be seriously considered. For the Galactic Center, the detection of gas streaming towards the center by Wollman et al (1977) pointed at a high mass concentration. The detection of high velocity stars presented first direct evidence for the presence of a SMBH (Eckart and Genzel, 1996, 1997; Ghez et al, 1998).

In the meantime, an impressive number of observations has yielded indirect evidence for the presence of black holes in the centers of many, if not all, bright galaxies (Ferrarese et al, 2006; Nayakshin et al, 2012). Galaxy evolution itself seems to be strongly connected to black hole evolution (Ferrarese et al, 2006; Ferré-Mateu et al, 2015; Kormendy and Ho, 2013). High masses of dark and very compact objects, i.e. most likely SMBHs (see section 3 in particular section (5), can be measured, and it seems possible to determine the spin of the black holes from X-ray measurements (e.g. Revnolds, 2014).

Kiloparsec- and parsec-scale jets are among the most prominently visible signatures of black hole accretion. Cosmic black holes, including the SMBH in Sgr A*, are not isolated in their environment. Instead, surrounding gas and stars also contribute to some extent to the gravitational field. Therefore, the classical sequence of Schwarzschild - Kerr - Reissner-Nordström - Kerr-Newman solutions for black holes in vacuum should be generalized to account for distributed material in their vicinity. This has been discussed by many authors in the context of exact solutions in GR; nonetheless, self-gravitating disks and rings are of particular interest, as such structures are detected in many objects (Karas et al, 2004). 
Galaxy collisions and the subsequent coalescence of binary black holes explain galaxy/black hole growth and black hole activity (Sesana et al, 2011; Bogdanović, 2015). Strong gravitational wave signals occur during the final phase of the merging process (see the detection of gravitational waves from binary stellar sources by the LIGO and Virgo collaborations Abbott et al, 2016) and might be detected with Pulsar Timing Arrays (e.g. Wyithe and Loeb, 2003; Roedig and Sesana, 2012; Pollney and Reisswig, 2011) in the future. Current efforts are also summarized in reviews by Berti et al (2015), Gair et al (2013), and Yunes and Siemens (2013). While the Pulsar Timing Array will detect the foreground of Gravitational Waves resulting from the incoherent superposition of inspiralling SMBH binaries well before merging (i.e., far from coalescence), LISA and eLISA7 will detect SMBHs at coalescence (e.g. The eLISA Consortium and Seoane, 2013). These observatories will have the highest sensitivity just in the mass range between $10^{5}$ to a few $10^{6} \mathrm{M}_{\odot}$, i.e., masses even below that of SgrA*.

Black holes play an increasingly important role in many areas of astrophysics. Therefore, more pressing seems to be the question: How good a case is it? With regard to collecting observational evidence closer and closer to the presumed event horizon it seems odd and intellectually dissatisfying to approach a zone of principal non-observability (see also comments on "underdetermination" in section 3.1). The wealth and complexity of the collected astrophysical observational data on the surroundings and properties of the central object are in sharp contrast to the rather simple expectations and predictions for the very central supermassive object. Yet, both regions are connected and need to form a consistent physical picture. It is essential to explain in detail the tools of physics at the photon sphere of an event horizon, and prepare for a consistent explanation of the wealth and complexity of the collected observational data describing the immediate vicinity of the central object.

\subsection{Working definition of a (supermassive) black hole}

A black hole may be described as a geometrically defined compact region of spacetime associated with a mass 8 . The gravitation is so strong that nothing can escape from inside the event horizon as defined in section 2.2. In this context, the no-hair theorem is often quoted which states that a black hole is fully described by only three externally observable classical parameters: mass, electric charge, and angular momentum 9 . Of all known SMBH candidates in the Universe, SgrA* at the center of the Milky Way is particularly well suited to test the no-hair theorem, because it is the closest of these candidates (see also comments on "baryonic hair" in section 5.4 and Johannsen (2016)). A SMBH differs from a stellar black hole simply by the amount of mass contained in it. For stellar black holes the mass is typically between 1.4 and a few 10 solar masses, whereas the SMBHs located in the nuclei of massive galaxies harbor millions (to billions) of solar masses. It is currently unclear how well the regime between the stellar and SMBHs is populated. It is technically difficult to find very good candidates for these intermediate-mass black holes (IMBHs) in the mass range between $10^{2} \mathrm{M}_{\odot}$ and less than about $10^{5} \mathrm{M}_{\odot}$ (Colbert and Mushotzkv, 1999).

\footnotetext{
7 LISA and eLISA are proposed Laser Interferometer Space Antennae; see https://www.elisascience.org/

8 Recent aspects regarding quantum properties of very small black holes (which are not subject of our article but are important to get a complete picture of the envisaged properties of back holes) are given in e.g. Christodoulou et al (2016); Barrau et al (2016); Haggard and Rovelli (2015); Rovelli and Vidotto (2014); Barrau et al (2014); Kiefer (2015).

9 The same mathematical formalism allows us to include the magnetic charge (a monopole) as the "fourth hair". However, this possibility is usually rejected on simple physical grounds - there are no magnetic monopoles found in nature so far.
} 
Ultra-luminous X-ray sources within the bulge and disks of galaxies or central objects in globular clusters are good candidates (e.g. Pasham et al, 2014; Wrobel et al, 2016; Earnshaw et al, 2016; Arca-Sedda, 2016). Kiziltan et al (2017) found an indirect evidence for an IMBH of about $2200 \mathrm{M}_{\odot}$ at the center of the globular cluster 47 Tucanae based on the dynamical state constrained by the timing data of millisecond pulsars. However, the inferred IMBH does not have a corresponding electromagnetic counterpart, which implies that most of the IMBHs may be not accreting enough to be bright in X-ray and radio domains, explaining the lack of direct detections. In the Galactic plane, the IMBHs can also be detected indirectly through either their velocity kicks imparted to the surrounding molecular clouds (Oka et al, 2016) or the synchrotron signal associated with the bow shocks in case they move supersonically (Wang and Loeb, 2014).

The simplicity of the definition given above may be complicated by the fact that black holes may have an angular momentum (i.e. a spin) which leads to the formation of an ergosphere (from $́$ e $\gamma o \nu$, which means "work" in Greek). In theory, it is possible to extract energy and mass from the ergosphere at the expense of the rotational energy of the black hole, which is also known as Penrose process or Blandford-Znajek effect for the explanation of the jet generation. The spin of the black hole also determines the radius of the closest stable orbit of a (test) particle around it. Within GR and the standard definition of an isolated black hole, exact solutions of Einstein-Maxwell equations are known to describe black holes in terms of only the three above-mentioned physical parameters: mass, spin, and electric charge (Chandrasekhar, 1983; DeWitt and DeWitt, 1973; Hawking and Israel, 1987). Astrophysically realistic solutions restrict also the mass of cosmic black holes by the mechanism and history of their formation. Natarajan and Treister (2009) show that there is an upper limit of $10^{10} M_{\odot}$ for astrophysical black holes. King (2016) continued in the analysis and derived a physical limit of $M_{\max } \approx 5 \times 10^{10} M_{\odot}$ to the mass of the supermassive black hole, above which the luminous accretion cannot proceed (the innermost stable circular orbit is larger than the inner radius of the accretion disk). Hence, the maximum mass is just an observational limit, since further accretion above it is non-luminous, i.e. it does not manifest itself as a quasar or active galactic nucleus. Hence, black holes above $M_{\max }$ are expected to be low-luminous and therefore difficult to detect.

The allowed range of charge and spin is limited; above that limit, the horizon ceases and a naked singularity (see below in section 2.2) emerges. In principle, a non-zero value of magnetic charge can be included; however, this possibility is usually neglected because of the (possible) non-existence of magnetic monopoles in the universe. When the black hole is embedded in a large-scale (cosmological) magnetic field, the standard assumption of asymptotic flatness must be abandoned and the notion of the event horizon has to be generalized (García Díaz, 1985; Esteban and Ramos, 1988). Nonetheless, other properties including the uniqueness theorems can be reformulated and maintained (Karas and Vokrouhlický, 1991).

\subsection{Black Holes in General Relativity}

The uniqueness theorems for black holes mentioned at the beginning of section 2 refer to stationary black holes, that is, asymptotic end states of a collapse. For the general situation of non-stationary and non-symmetric black holes, a more precise definition is needed. The exact mathematical form is involved and can be found in standard textbooks such as Wald (1984) or Poisson (2004); for a semi-popular account, see Hawking and Penrose (1996). It captures the physical idea of a "region of no escape", that is, a region of spacetime which will never become part of an external observer's past. The boundary of this region is called event horizon, more 
precisely, future event horizon, because one assumes the usual arrow of time (as given by the Second Law of thermodynamics) to hold 10

A future event horizon thus gives the boundary of the spacetime region (called black-hole region) from which nothing, not even light, can ever reach other spacetime regions. It must be emphasized that the whole future evolution must be known in order to specify the location of the event horizon; the event horizon is a threedimensional object (two spatial and one lightlike dimension). But is the event horizon really the most adequate concept for describing observations, as indicated, for example, by the name of the project "Event Horizon Project" (EHT; see introduction)? When observing a black hole such as the SMBH in the Galactic center now, we cannot know of any amount of matter that will fall into this black hole in the future and will lead to an increase of mass and, consequently, of an increase of the size of its event horizon. We thus need alternative notions which are of a more local nature. Such notions are, in fact, used 11. The most important one for our case is the notion of an apparent horizon. For its definition, one considers the boundary between the region where emitted light can reach infinity and the region where it cannot. This three-dimensional boundary is called "trapping horizon", and its two-dimensional intersection with a space-like surface (that is, with a surface of constant time), is called apparent horizon (Wald, 1984; Poisson, 2004). This is the concept usually used in numerical relativity. The interior of the trapping horizon is characterized by the presence of "trapped surfaces"; these are two-dimensional surfaces for which both in- and outgoing light rays propagate inwards 12 . The word "shadow" is used here to describe a region close to the black hole towards which the emission is greatly suppressed due to relativistic effects (likelight bending and boosting due to light abberation). A first formal approach as how to calculate photon paths near black holes in the context of a shadow was given by J.L. Synge in 1966 (Synge, 1966). The first image of an accretion disk around a black hole including a shadow was given by Luminet in 1979 (Luminet, 1979). Possible shapes and geometrical arrangements of the shadow are discussed, e.g., in Bardeen (1973); Grenzebach et al (2015); Abdolrahimi et al (2015).

For stationary black holes of mass $M_{\bullet}$, the apparent horizon coincides with the (time slice of the) event horizon. In the simplest case of the Schwarzschild solution, the horizon size is given by the Schwarzschild radius

$$
R_{\mathrm{S}}:=\frac{2 G M_{\bullet}}{c^{2}}
$$

for the Kerr black hole, the horizon is located at

$$
R_{\mathrm{Kerr}}:=\frac{G M_{\bullet}}{c^{2}}+\sqrt{\left(\frac{G M_{\bullet}}{c^{2}}\right)^{2}-a^{2}} .
$$

Quite generally 13 the apparent horizon lies within the event horizon or coincides with it.

The presence of trapped surfaces strongly indicates the formation of a singularity, that is, a region where Einstein's equations (and other equations) no longer hold. This is the content of the famous singularity theorems (Hawking and Penrose, 1996).

\footnotetext{
10 Because GR is time-symmetric, there is also a past event horizon. It is the boundary of a so-called "white-hole region", that is, a spacetime region into which nothing can enter and which thus will never become part of an external observer's future. White holes are usually excluded for thermodynamic reasons, analogously to the exclusion of advanced potentials in electrodynamics, cf. Zeh H.D (2007) for a careful and detailed discussion of these issues.

11 Hawking has recently claimed that only apparent horizons exist (Hawking, 2014)

12 For the observers of the black hole's "shadow", it is perhaps the photon sphere that is the most important quantity.

13 Provided the so-called "null energy condition" holds
} 
Singularities are predicted to occur in the interior of black holes. Strictly speaking, their occurrence signals the need for a more fundamental theory that replaces GR under such extreme conditions. It is generally expected that this theory is a quantum theory of gravity, see the next subsection.

It is an interesting and still to some extent open question whether an event horizon forms in a realistic gravitational collapse. If not, the singularity (or its replacement in a more fundamental theory) will be seen from outside, leading to a loss of predictability. Because of the disturbing consequences of such "naked singularities", Roger Penrose came up in 1969 with his cosmic censorship conjecture, which loosely states that "Nature abhors naked singularities" (see also comments on "underdetermination" in section 3.1) or, more precisely, in the formulation given in Wald (1984) , p. 304:

All physically reasonable spacetimes are globally hyperbolic, i.e., apart from a possible initial singularity (such as the "big bang" singularity) no singularity is ever "visible" to any observer.

Presently, it seems that this conjecture holds in a generic initial condition (excluding conditions of high symmetry). If it were violated and naked singularities did exist in Nature, this would have tremendous consequences for astrophysics. The value of black holes (or black-hole candidates) for the test of GR and possible generalizations can hardly be overestimated (Yagi and Stein, 2016). This holds, in particular, for the Milky Way's SMBH, which can be viewed as an optimal "laboratory" for GR (Johannsen, 2015).

\subsection{Black Holes and Quantum Theory}

We have seen that stationary black holes are fully characterized by just three parameters. This reminds one of the situation in thermodynamics where the macroscopic properties of a gas are fully characterized by few parameters such as pressure, volume, and entropy. Surprisingly, it was found in the 1970s that black holes are thermodynamic systems; they obey complete analogies to all four laws of thermodynamics (see e.g. Hawking and Penrose (1996) or Kiefer (1999) for an introduction). They possess, in particular, a temperature (the Hawking temperature) and an entropy (the Bekenstein-Hawking entropy), which can only be interpreted if quantum theory is taken into account. In the case of a Schwarzschild black hole, the Hawking temperature is given by

$$
T_{\mathrm{BH}}=\frac{\hbar c^{3}}{8 \pi k_{\mathrm{B}} G M_{\bullet}} \approx 6.17 \times 10^{-8}\left(\frac{M_{\odot}}{M_{\bullet}}\right) \mathrm{K} .
$$

This leads to a finite lifetime for the black hole with an order of magnitude estimate

$$
\tau_{\mathrm{BH}} \sim 10^{65}\left(\frac{M_{0}}{M_{\odot}}\right)^{3} \text { years, }
$$

where $M_{0}$ is the initial mass. The black hole becomes "hot enough" for quantum effects to be observable only near the final evaporation phase, which for astrophysical black holes only occurs in a far future. (For the Galactic Center black hole, the lifetime is of the order of $10^{84}$ years!) Nevertheless, there is also an astrophysical interest in the study of Hawking evaporation. First, small black holes may have formed in the early Universe and may evaporate today. Such "primordial black holes" may even contribute to the dark matter in the Universe (Blais et al, 2002) and produce gravitational waves (Bird et al, 2016). Second, according to recent speculations, quantum effects may potentially become relevant near the horizon of 
a macroscopic black hole; for example, in the form of a "firewall" (Calmet, 2015); see also Giddings (2014). It is an intriguing question whether such effects, if present, could be observed with the EHT.

Quantum effects of black holes play a crucial role in the search for a quantum theory of gravity (Kiefer, 2012). Such a theory will give a complete description of black hole evolution and, in particular, of the final evaporation phase. For the latter, only very rudimentary models are available; see, for example, Kiefer et al (2009). The theory should also provide one with a microscopic explanation of the Bekenstein-Hawking entropy. Whether observations of the Galactic Center black hole can provide hints in this direction, is an open issue. Let us finally emphasize that quantum effects are also essential for concepts of black hole alternatives (see section (5), like the "fermion ball", "boson star", and "grava-stars" scenarii, as well as the phenomenon of macro-quantumness.

\section{Philosophical Concepts}

Although the observational evidence for SgrA* being a SMBH is ever increasing, one has to ask the question: At what point can we be sure that it really is a SMBH? Before we review the evidence in detail it is necessary to deal with some conceptual issues. First, what does it mean for a black hole to be real, or to exist? The philosophy of science allows us to use different approaches to deal with the problem of showing the reality or existence of SMBHs. Here we follow a two stage approach: First, we have chosen the concept of "(Anti)Realism and Underdetermination" as one line of arguments that is close to physics. Observational astrophysics is a science of measuring and quantifying entities, which are subject to questions of (anti)realism and underdetermination (see section 3.1). These are concepts that predominantly help us to show the validity of theories. Second, as we are interested in possibilities of probing the existence of SMBHs we have chosen a line of arguments that requires the causation as an indispensable element if one wants to talk about the reality and/or existence of entities. The linkage between these two lines or arguments is laid out in Appendix B

\section{1 (Anti)Realism and Underdetermination}

On one hand, philosophy of science has recognized the problem that the evidence from data may never be able to sufficiently discriminate one theory from another, rivaling theory. This problem of underdetermination is usually referred to as the epistemological problem of the indeterminacy of data to theory. On the other hand, "entity realism" is not committing itself to judge on the unique validity of scientific theories, but relies on repeatable routinely observable effects that provide or represent sufficient evidence to reliably support the validity of a theory. This is reflected in an often mentioned quotation by Ian Hacking: "if you can spray them, then they are real" (Hacking, 1983, page 24). This statement can be translated into: If you can use entities to manipulate others, then we have sufficient evidence for their reality. Ian Hacking, as the main proponent of this formulation of entity realism, was referring to an experiment he observed in a Stanford laboratory, where electrons and positrons were sprayed onto a super-conducting metal sphere. To be used as an instrument in a manipulation of other systems, this entity realism presupposes a quantitative precise causal profile (see e.g. Hoefer and Smeenk, 2016) in order to bring about the effects in question. If the effect is successfully brought about, then one has sufficient evidence for the claim that there is something with this particular profile. 
Studying black holes as research objects faces a severe problem, as global properties of a single, possibly uniquely defined object, may be systematically underdetermined (e.g. Cleland, 2002; Anderl, 2015, 2016; Smeenk, 2005) That makes it difficult to find the true (responsible) causes for an observational result. This underdetermination was identified by Hacking as a "methodological otherness" in comparison with other realistic scientific disciplines (Hacking, 1983; Sandell, 2010).

Underdetermination has a theoretical and an experimental side: The theory may not be fully complete and only highlight certain properties. The concept of underdetermination may indeed be particularly relevant for black holes as astrophysical objects, for which - according to Hacking - we cannot make a claim to realism, implying a sort of antirealism, as one may state that due to observational insufficiencies we have no access to a reality even if it may exist. As an example specific to black holes, the whole future evolution must be known in order to determine the location of the event horizon (see also comments on "naked singularities", and "firewalls" in section 2.1 and 2.2, which will be similarly difficult to observe and hence are likely to be subject to underdetermination). Hence, at least in this case, underdetermination may be included as an inherent property of the theory. In addition, the observations may be not unique enough to clearly distinguish one possible realization of an object from another, since the interpretation of the observations may just be based on a restricted set of theoretical predictions. In the case of experiments (see e.g. Franklin, 2016; Galison, 1987), however, one has the chance to fight (i.e. minimize or even remove the effect of) underdetermination by increasing the observational evidence and combining various procedures that approach the problem with different methods or instrumental efforts.

Currently, it is observationally quite possible to decide uniquely on the presence of very compact and massive objects based on astrophysical observations. However, it is not quite possible to differentiate between different theories of compact objects that predict similar observational facts under the boundary condition of a minimal use of auxiliary input (i.e. theoretical stability arguments and rejection of the existence of certain particles; see section 5). This means that, despite theoretical and experimental progress, it still remains difficult to resolve problems of underdetermination. This is reflected in the fact that the attempt of an observational proof of a theory involves and entails auxiliary hypotheses, available instruments, and background assumptions (e.g. Laudan and Leplin, 1991).

An important feature that helps to minimize underdetermination is the application of independent experimental observational methods or channels. In this respect the newly established possibility to measure gravitational wave signals is an essential point (e.g. Berti et al, 2016). While gravitational wave astrophysics is in its infancy, the possibility of carrying out gravitational wave spectroscopy cannot be ignored when discussing underdetermination. The cooperative efforts coming from both "classical", electromagnetic observational astrophysics and the new gravitational wave astrophysics will substantially contribute to our future picture of supermassive black holes. However, Cardoso et al (2016) point out that very compact objects with a light ring will display similar ringdown signals as black holes, implying that universal ringdown waveforms indicate the presence of light rings, rather than that of horizons. Hence, it is only that high precision measurements of the late-time ringdown signals may allow us to rule out exotic alternatives to black holes.

The situation depicted above is usually described in the framework of a "contrastive underdetermination", meaning that for each set of observational evidences there may exist several different but equally empirically testable theories. Stanford (2013) points out that this "holist underdetermination" implies that a theory can be adopted to new sets of observational results. This is particularly true, as these results do not solely depend only on observational facts that test the theoretical 


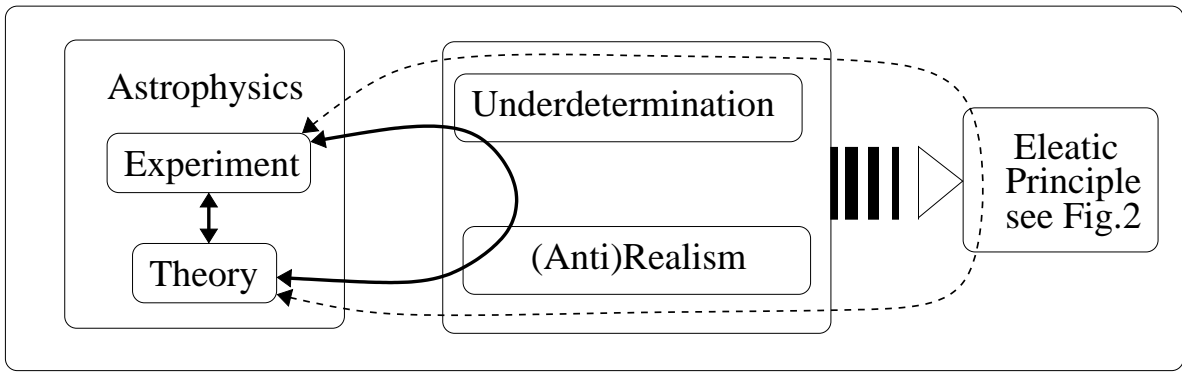

Fig. 1 Linkage between experiment and theory interpreted via the concept of realism and underdetermination finally allowing us to discuss the question of realism and existence in the framework of causation, making use of a form of the Eleatic Principle.

claims, but also depend on many other auxiliary hypotheses, which may be right, or wrong or which may be right by themselves but cannot be fully used in the context of a test.

In the case of supermassive black holes this may lead to an "abductive justification" for black hole proofs, i.e., the assumption that a theory might very likely be true if it is the currently best explanation of the observational facts (including a set of auxiliary information). This asks for a strategy that tries to reduce the holistic underdetermination by investigating different sets of auxiliary assumptions for their influence on the theoretical prediction and the interpretation of observational evidences. This is our main intention in sections 4, 5] and 7.

\subsection{The Involvement of Causality}

The discussion on "Realism" and "Underdetermination" targets mainly the consistency and repeatability of observations and in how far they provide sufficient evidence to reliably support the validity of a theory. As in all experiments discussed below it is never the supermassive black holes themselves that are observed (with the gravitational radiation originating closest to the black holes; see section 4.13) but only objects that interact with it, e.g. gas, stars, light. Hence, the evidence to reliably support the validity of a theory comes in through causality described by the theory. Therefore, for both theory and observations there are direct links from the concepts of "Realism" and "Underdetermination" to the concept of the causality. More detailed information on how this linkage may be described is given in Appendix B. Hence, if the problem of underdetermination can be minimized, one is approaching the situation in which the causal relevance and the indispensability of black holes is strengthened. More detailed information on this is given in Appendix C, in which we describe the Eleatic Principle as a possible causal criterion that may be used (Colyvan, 1998).

The overall concept is shown in Fig 1, showing the linkage (the thin continuous black lines) between experiment and theory that can be discussed using both, the concept of realism and underdetermination. If underdetermination can be minimized, we may approach (as shown by the dashed thick bar) a situation in which we can start discussing (shown by the thin dashed black curved line) the question of underdetermination, realism and existence in the framework of causation, justifying the usage the "rounded out" version of the Eleatic Principle (see Appendix (C). Usually a causal criterion is implemented as a test that must be passed by logical statements or objects in order to be accepted within a scientific context, that is, the study of the nature of being, becoming existent, existence, or reality. 


\section{Colyvan's rounded out version of the Eleatic Principle}

- for reasons of symmetry and theoretic virtue

- allowing for entities that are causally idle but causally relevant

- hence, including the Eleatic Principle relying mainly on causal entities and balancing the unsatisfactory justification attempts

\section{Classical Eleatic Principle}

as a logical test for causality that must be passed before acceptance within a scientific onthology.

The principle is mainly relaying on causally active entities but leads to largely unsatisfactory justification attempts

Fig. 2 The classical Eleatic Principle in relation to the "rounded out" version proposed by Colyvan (1998).
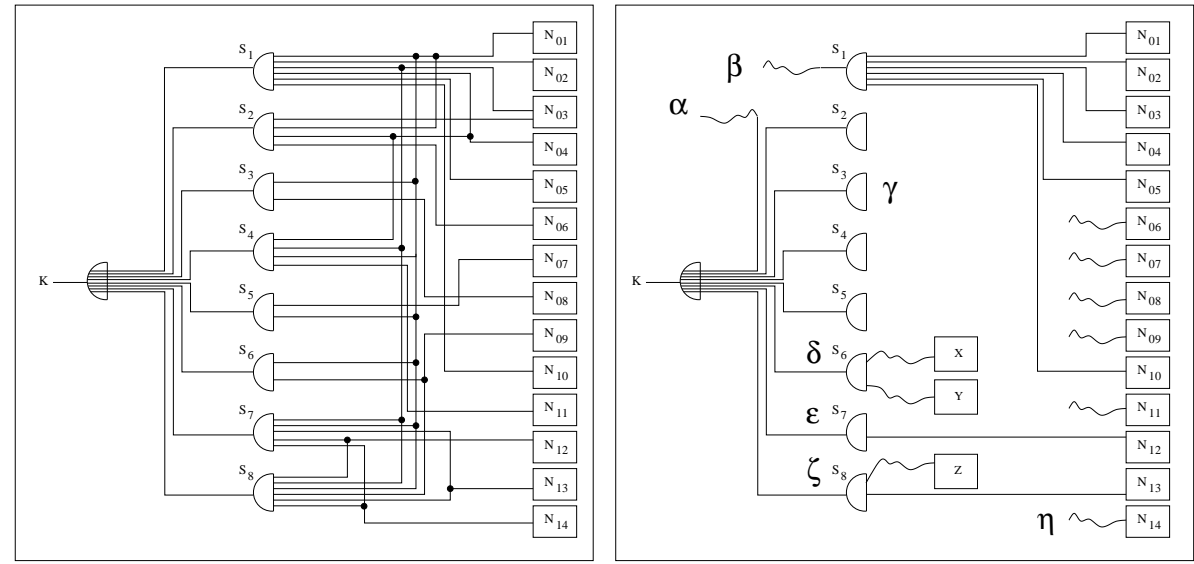

Fig. 3 Left: Epistemological process following the Eleatic Principle requesting logical standards of clarity as criteria of truth - here represented by the usage of logical gates. In section 7 this network is used and described in detail. Here appropriate necessary conditions $N_{i}$ are combined to sufficient conditions $S_{k}$ and a combined conclusion $K$ following logical standards (Here $i$ and $k$ are running indices). Right: Depiction of how criteria simply based on sensual experiences may effect the epistemological process. In the case of astrophysical research, we may consider data, i.e. observational or computational results, as sensual experiences. If we do not follow causal criteria, then the usage of these data as sensual experiences does not stringently require the usage of logical standards to link them. We labeled a few cases that demonstrate what could go wrong: $\alpha$ : the claim of existence is a claim not supported by data; $\beta$ : a combination of correct necessary conclusions is (accidently) not considered; $\gamma:$ a sufficient condition is claimed and not supported as reliable data; $\delta$ : a sufficient condition is wrongly concluded from unrelated conditions; $\epsilon$ : a sufficient condition is (accidently) claimed from an insufficient number of necessary conditions; $\zeta$ : a sufficient condition is (accidently) claimed from a combination of unrelated conditions and related necessary conditions; $\eta$ : properly related necessary conditions are overseen and not used. 
While there is some debate about whether the causal criterion provides a necessary condition for existence or reality (e.g. the reality of numbers in mathematics is disputable), there is much less controversy that it provides a sufficient condition for existence or reality. There is no question that there is something at the center of the Milky Way in virtue of there being something acting on the nearby stars etc. . The essential question is whether this something is a black hole. Under what conditions can we decisively answer this question? For comparison we give in Appendix $\mathrm{A}$ a historic example for establishing existence claims in the case of atoms and molecules.

Hence, in order to identify a specific entity, it is not sufficient to know that there is something that has some sort of effect. In order to establish a specific existence claim we need to establish that the entity in question manifests itself in very specific ways, so as for instance to put constraints on the values of experimentally accessible variables. An essential presupposition for establishing a specific existence claim is thus to have a sufficiently precise characterization of the entity in question (as done in section 2.1), a characterization in terms of the causal/nomological relations it enters into. In order to get clarity about measuring and combining these relations we follow a formal logical approach outlined in the next section.

\subsection{Formal Logical Approach}

There is a broad spectrum of different observational entities that either result from different instrumental methods and/or refer to different aspects of theory. Naturally this results in a similarly broad spectrum of combinations of these entities to (observationally and theoretically) support the validity of the black hole theory and lead to the acceptance of the existence of supermassive black holes which is at the focus of this contribution. To provide clarity in the approach we need to combine these entities in a formal logical way. Such a treatment then fully accounts for the logical standards following the a causal criterion (e.g. the Eleatic Principle) opposed to criteria simply based on sensual experiences as demonstrated in Fig. 3. Probing the existence of entity $K$ in such a formal way, $K$ can be expressed via the concept of necessary and sufficient conditions for something being fulfilled or true - within the philosophical concepts laid out in the previous sections. In the following, we have to assume that $\nu$ and $\mu$ are natural numbers. If $N_{\mu}$ are necessary conditions and if we use the universal quantifier, we can then express the logical value of $S$ via:

$$
S \Longrightarrow \forall \mu N_{\mu} \Longleftrightarrow N_{1} \wedge N_{2} \wedge N_{3} \wedge \ldots \wedge N_{\mu}
$$

The question may remain if all necessary conditions for the proof of existence are known and fulfilled and if the result then is indeed a sufficient condition for the existence.

Similarly if $S_{\nu}$ are sufficient conditions we can use the existential quantifier and can then express the logical value of $K$ via:

$$
K \Longrightarrow \exists \nu S_{\nu} \Longleftrightarrow S_{1} \vee S_{2} \vee S_{3} \vee \ldots \vee S_{\nu}
$$

Here, the question remains if there are several sufficient conditions and if they are all of equal quality, i.e., is one better or more convincing than the other to prove the existence even though they both can in principle be true (or false). There may also be the case that a sufficient condition may be represented via the concatenation of a subset of two or more necessary conditions, such as:

$$
S_{i} \Longrightarrow \forall \lambda N_{\lambda}
$$


with $1 \leq i \leq \nu$ and $1 \leq \lambda \leq \mu$. This allows us now to formulate a formal logic description of how to combine the necessary conditions in order to test if SgrA* can be accepted as a SMBH. In general, $\nu$ different subsets of a number of $\mu(\nu)$ necessary conditions may lead to sufficient conditions with the logical value of $K$ expressed via:

$$
K \Longrightarrow \exists \nu S_{\nu} \Longleftrightarrow \exists \nu \forall \mu(\nu) N_{\nu, \mu(\nu)}
$$

with

$$
\begin{aligned}
\exists \nu \forall \mu(\nu) N_{\nu, \mu(\nu)} \Longleftrightarrow & \left(N_{\kappa_{1,1}} \wedge \ldots \wedge N_{\kappa_{1, \mu(1)}}\right)_{1} \vee \\
& \left(N_{\kappa_{2,1}} \wedge \ldots \wedge N_{\kappa_{2, \mu(1)}}\right)_{2} \vee \ldots \\
& \vee\left(N_{\kappa_{\nu, 1}} \wedge \ldots \wedge N_{\kappa_{\nu, \mu}(\nu)}\right)_{\nu} .
\end{aligned}
$$

Here, $\nu$ is the number of combinations of necessary conditions to form a sufficient condition while $\mu(\max )$ is the total number of necessary condition available. Then $\mu(\nu)$ is the number of necessary conditions for each of these $\nu$ possible combinations with $\mu(\nu) \leq \mu(\max )$. Then sufficient condition $S_{i}(1 \leq i \leq \nu)$ is a combination of necessary conditions with $N_{\kappa_{\lambda, \mu(\lambda)}}$ being the $\kappa_{\lambda, \mu(\lambda)}$-th element of the set of all necessary conditions for the $\nu$-th combination.

If for all combinations (i.e. for all $\lambda$ with $1 \leq \lambda \leq \mu$ ) e.g. one common necessary condition $C=N_{\kappa_{\lambda, \mu(\lambda)}}$ could be declared as a common necessary condition, this could of course be isolated,

$$
\begin{aligned}
K \Longrightarrow & \exists \nu \forall \mu(\nu) N_{\nu, \mu(\nu)} \\
\Longleftrightarrow & C \wedge\left[\left(N_{\kappa_{1,1}} \wedge \ldots \wedge N_{\kappa_{1, \mu(1)}}\right)_{1} \vee\right. \\
& \left(N_{\kappa_{2,1}} \wedge \ldots \wedge N_{\kappa_{2, \mu(1)}}\right)_{2} \vee \ldots \vee \\
& \left.\left(N_{\kappa_{\nu, 1}} \wedge \ldots \wedge N_{\kappa_{\nu, \mu(\nu)}}\right)_{\nu}\right] .
\end{aligned}
$$

However, for simplicity it is easier to treat the complete sets of necessary conditions, even though a more compact notification may be possible. In section 7, we will give examples for different combinations for the case of the SMBH at the center of the Milky Way.

\section{Evaluation of observationally based results}

In the following, we give a description and evaluation of observationally based facts that either point at the existence of a $\mathrm{SMBH}$ or are interpreted in the context of such an object. Those include the distance, mass, size, spin, orientation, spectrum, and variability, as well as the possibility of a jet, horizon, shadow, or a charge, to be described in sections 4.1 to 4.12 . On the left side of Fig, 4 we show the relation between some of these quantities. The right side of Fig 4 shows an infrared image of the central stellar cluster at the center of which $\mathrm{SgrA}^{*}$ is located.

\subsection{Distance}

The distance to the center of the Milky Way and hence to the location of the SMBH is essential, for it allows us to transform the observed angular measurements into linear scales 14 It position in the sky has been determined very accurately via VLBI

\footnotetext{
14 Angular scales in this context are mostly given in fractions of arcseconds, i.e., milliarcseconds (mas) or microarcseconds ( $\mu$ as). While the physical distance to the Galactic Center is given in kilo-parsecs (kpc; one parallactic seconds or $1 \mathrm{pc}=3.09 \times 10^{16} \mathrm{~m}$ ) the physical scales at the Center are given in light years $\left(\mathrm{ly}=9.46 \times 10^{15} \mathrm{~m}\right.$ ) or parsecs (parallactic seconds or $1 \mathrm{pc}=3.3 \mathrm{ly}$ ) or fractions thereof, i.e., milli-parsecs $(\mathrm{mpc})$ of micro-parsecs $(\mu \mathrm{pc})$. On even smaller scales, the sizes are given in astronomical units $(\mathrm{AU}=149,597,871 \mathrm{~km})$ or in Schwarzschild radii $R_{s}$ that are linked to the black hole mass $M$ via $R_{s}=\frac{2 G M}{c^{2}}$, where $G$ is the gravitational constant and $c$ is the speed of light.
} 
measurements (Menten et al, 1997; Reid et al, 2003b). 15 There are two often used methods to derive the distance to the black hole at the center of the Milky Way. The classical method uses globular clusters - or in general - stellar populations, determines their distances or kinematic properties and then infers symmetry considerations to derive the center of the Milky Way. Under the assumption that the $\mathrm{SMBH}$ is located at this center, it then can be taken as the distance to the black hole radio counterpart SgrA*. The second method uses orbits of a single star (mostly S2) or several central stars and combines proper motions and radial velocity information to derive the distance of these stars orbiting the SMBH.

Genzel et al (2010) compiled all distance estimates to the Galactic Center from the beginning of the last century until 2010. Malkin (2013) carried out a thorough and detailed study of the Galactic Center distance measurements between 1990 and 2010. Both studies seem to suggest that the uncertainty in the distance estimate apparently decreases as a function of time. In Fig 5 we summarize the recent efforts to determine the distance to SgrA*.

From symmetry considerations of the distribution of recently updated globular cluster distances, Francis and Anderson (2014) estimate the distance to the Galactic Center as $\mathrm{R}=7.4 \pm 0.28 \mathrm{kpc}$. However, typically larger values are obtained if more information is included in the distance derivations. From the distribution of several thousand OB-stars within $1 \mathrm{kpc}$ of the Galactic Center, Branham (2014) derived a distance to it as $\mathrm{R}=6.72 \pm 0.39 \mathrm{kpc}$. Using the characteristics of the stellar populations and the metallicity distribution in the Galactic bulge, Vanhollebeke et al (2009) derived a distance to the Galactic Center of $\mathrm{R}=8.7 \pm 0.71 \mathrm{kpc}$. Majaess et al (2009) use the distribution of $\delta$-Cepheids and find $\mathrm{R}=7.7 \pm 0.7 \mathrm{kpc}$. Dékánv et al (2013) combined optical and near-infrared data of known RR Lyrae (RRL) stars in the bulge and studied the spatial distribution and distances of 7663 RRL stars. The authors obtained a distance to the Galactic Center of $\mathrm{R}=8.33 \pm 0.15 \mathrm{kpc}$. Dékány et al (2013) also report a different spatial distribution of the metal-rich and metal-poor stellar populations suggesting that the Milky Way may have a composite bulge. Depending on the nature of this bimodal distribution, this may also indicate that more indirect methods of deriving the distance to the Galactic Center may be affected by systematic uncertainties. For the central 0.5 pc of the Milky Way nuclear star cluster Do et al (2013) present three-dimensional kinematic observations of stars using adaptive optics imaging and spectroscopy from the W.M.Keck telescopes. Solving simultaneously for the de-projected spatial density profile, cluster velocity anisotropy, the black hole mass, and distance to the Galactic Center they find a value of $\mathrm{R}=8.92 \pm 0.56 \mathrm{kpc}$. In combination with the orbital data of $\mathrm{S} 2$ an improved value of $\mathrm{R}=8.46 \pm 0.40 \mathrm{kpc}$ is obtained.

In the mean time, extensive astrometric and spectroscopic observations of the central high velocity stars have been carried out using the ESO $8.2 \mathrm{~m}$ mirror Very Large Telescope dishes and the W.M.Keck $10 \mathrm{~m}$ telescopes (Schödel et al, 2002; Eisenhauer et al, 2003; Horrobin et al, 2004; Ghez et al, 2008; Gillessen et al, $2009 \mathrm{a}, \mathrm{b})$. The most recent quite consistent combined estimates of mass and distance have been obtained by Boehle et al (2016) with $M=4.0 \pm 0.2 \times 10^{6} M_{\odot}$ and $7.9 \pm 0.2 \mathrm{kpc}$ based on a combined orbital fit of S2 and S38 data, and by Parsa et al (2017) with $M=4.29 \pm 0.02 \times 10^{6} M_{\odot}$ and $8.25 \pm 0.02 \mathrm{kpc}$ based on a combined orbital fit of three stars closest to SgrA*: S2, S0-102 and S38. From Fig 5 we see that the measurements (as a function of time) started off from higher values of up to $10 \mathrm{kpc}$ and have now settled to a value of about $8 \mathrm{kpc}$ with total uncertainties of the order of $0.2 \mathrm{kpc}$.

$\overline{15 \text { Values from Menten et al }}$ (1997) with corrections by Reid et al (2003b): (J2000) 

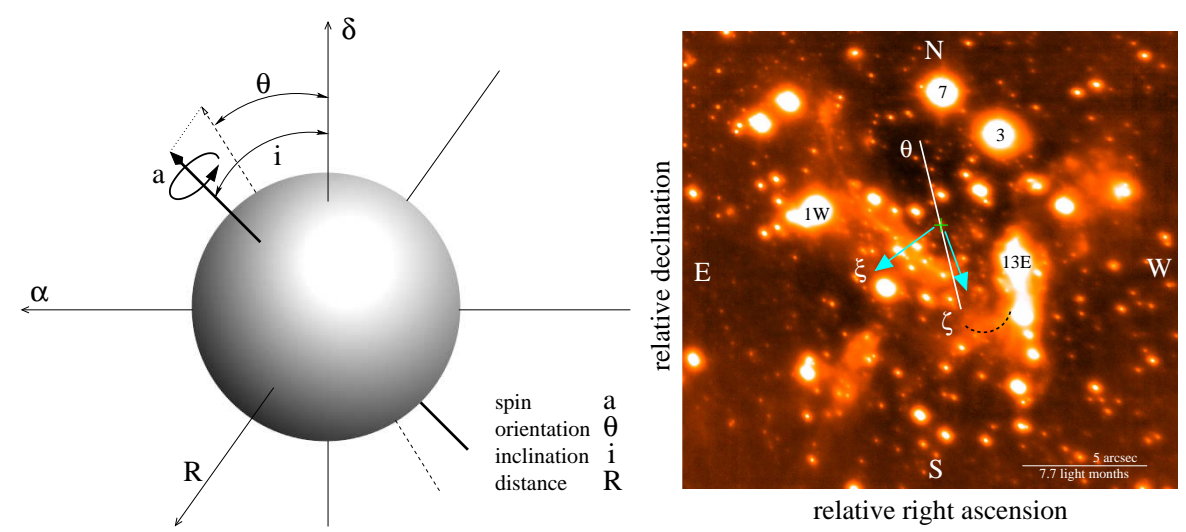

Fig. 4 Left: Relation between the observational quantities spin, orientation, inclination and distance of the black hole. The sphere depicts the Schwarzschild horizon of the black hole. The declination and right ascension axes are indicated by the symbols $\delta$ and $\alpha$, respectively. The dashed line represents the projection of the spin axis onto the plain of the sky. Right: An image of the central stellar cluster at $3.8 \mu \mathrm{m}$ wavelength (taken by some of the authors with the NACO instrument at the European Southern Observatory's Very Large Telescope) as it is surrounding the position of the SMBH SgrA* indicated by a green cross. The angular scale and the corresponding length scale at the location of the Galactic Center is given in the lower right corner of the image. Some bright stars (IRS1W, IRS3, IRS7 and IRS13E) are labeled. Possible geometrical orientation of the SgrA* system derived from near-infrared polarization measurements by Shahzamanian et al (2015a) is indicated by the white line labeled with the letter $\theta$. The blue arrow labeled with the letter $\xi$ indicates the orientation of a putative jet. The blue arrow labeled with the letter $\zeta$ indicates the orientation of a putative wind from $\mathrm{SgrA}^{*}$ into the mini-cavity shown as a dark dashed line.

\subsection{Mass}

The mass is one of the fundamental quantities that determine the nature of a black hole. Gaseous and stellar probes can be used to determine this quantity. Through observations of the $12.8 \mu \mathrm{m}$ NeII line emission from the mini-spiral in the Galactic Center stellar cluster Wollman et al (1977) revealed an enclosed mass of $4 \times 10^{6} \mathrm{M}_{\odot}$. However, at this time it was unclear how much the stellar cluster would contribute to the enclosed mass. These measurements were also conducted using ionized gas as mass probes. Hence pressure gradients and magnetic field could have influenced the result in addition. Compact point masses are much better probes for a gravitational potential, i.e., a mass measurement.

Krabbe et al (1995) report the first results of an extensive new study of stellar radial velocity measurements of the Galactic Center stellar cluster. The authors have measured the radial velocity dispersion of 35 stars with distances of less than 12 " from Sgr A* as $154 \pm 19 \mathrm{~km} \mathrm{~s}^{-1}$. This results in a mass estimate of about $3 \times 10^{6} \mathrm{M}_{\odot}$ within $0.14 \mathrm{pc}$ of the dynamic center. The first stellar proper motion measurements were reported by Eckart and Genzel (1996), Eckart and Genzel (1997), and Ghez et al (1998). Here, the authors present proper motion of 39 stars located between 0.03 and 0.3 pc from the Galactic Center. These motions indicate the presence of a central dark mass of $2.45 \pm 0.4 \times 10^{6} \mathrm{M}_{\odot}$ located within $0.015 \mathrm{pc}$ of the compact radio source Sgr A*. This compares well with the mass of $2.45 \pm 0.4 \times 10^{6} \mathrm{M}_{\odot}$ derived by Ghez et al (1998) from proper motions within the same region. Combining the first stellar orbital acceleration measurements from W.M.Keck data (Ghez et al, 2002) and from SHARP/NTT data Eckart et al (2002) apply a first order projection correction and derive a mass of $\mathrm{M}_{a c c}=(5 \pm 3) \times 10^{6} \mathrm{M}_{\odot}$. This estimate is consistent with the enclosed mass range of $(2.6-3.3) \times 10^{6} \mathrm{M}_{\odot}$ obtained by Genzel et al (2000) from radial and/or proper motion velocities of a homogenized sample of sources. 


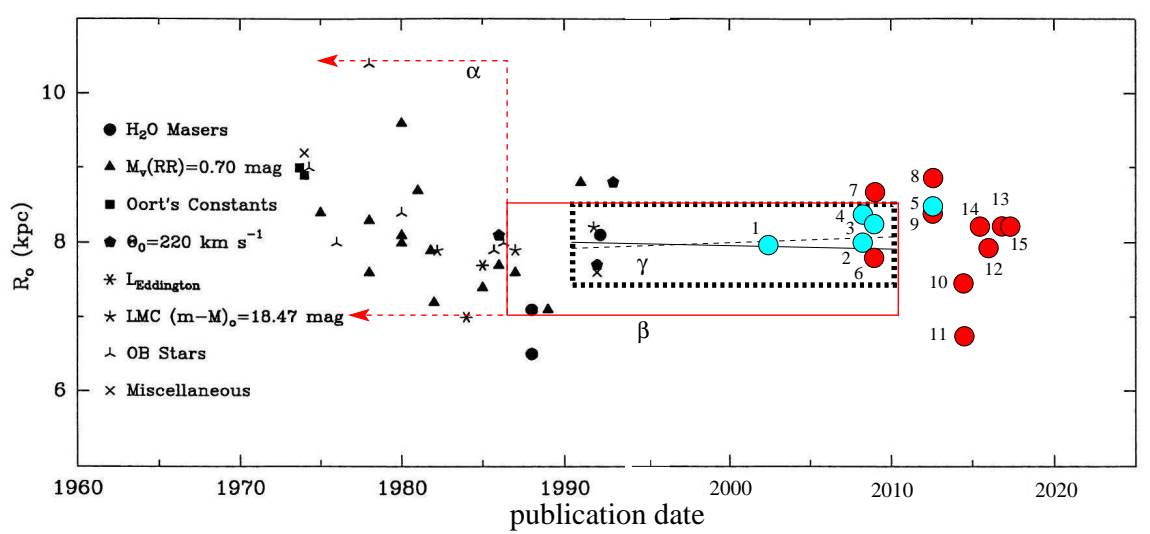

Fig. 5 Distance to the Galactic Center: Black symbols and comments are taken from Reid (1993). The bulk of the data presented by Genzel et al (2010) is located in region $\alpha$ and $\beta$. The data used by Malkin (2013) is mostly located in region $\gamma$. The solid and dashed lines in region $\gamma$ correspond to the weighted and unweighted versions of the calculated trends found by Malkin (2013). New and recent data points based on stellar orbital analyses are shown by turquoise filled circles and labeled: 1: Horrobin et al (2004); Eisenhauer et al (2003); Schödel et al (2002); 2: Ghez et al (2008); 3: Gillessen et al (2009a); 4: Ghez et al (2008); 5: Do et al (2013). Distance estimates based on stellar distributions in the global stellar cluster or Milky Way bulge are shown by red filled circles and labeled: 6 Majaess et al (2009); 7: Vanhollebeke et al (2009); 8: Do et al (2013); 9: Dékány et al (2013); 10 Francis and Anderson (2014); 11 Branham (2014); 12 Boehle et al (2016); 13 :Parsa et al (2017).

Combining NTT and VLT data for the S2 orbit several authors (Schödel et al, 2002; Horrobin et al, 2004; Eisenhauer et al, 2003) find $\mathrm{M}=3.7 \pm 1.5 \times 10^{6} \mathrm{M}_{\odot}$.

From three-dimensional stellar data within the central 0.5 pc of the Milky Way, Do et al (2013) derive a SgrA* mass of $\mathrm{M}=5.76_{-1.26}^{+1.76} \times 10^{6} \mathrm{M}_{\odot}$. Based on more than a decade of astrometric measurements of stellar orbits in the central half parsec, Ghez et al (2008) find a mass of $\mathrm{M}=4.5 \pm 0.4 \mathrm{M}_{\odot}$ under the assumption that the $\mathrm{SMBH}$ is at rest with respect to the stellar cluster. Gillessen et al (2009a) derives from the combined W.M.Keck and VLT data set a black hole mass of $\mathrm{M}=4.30 \pm$ $0.50 \times 10^{6} \mathrm{M}_{\odot}$.

In Fig, 6, we summarize the efforts to determine the mass of $\operatorname{Sgr} A^{*}$. Historically, the problem was to disentangle determinations of the black hole mass from potential contributions of the stellar cluster surrounding it. The graph shows that the initial mass estimate by Wollman et al (1977) was already quite close to the results obtained using the stars. There is also a clear trend for a mass increase as a function of time visible. While the early measurements using stellar radial velocities and proper motions all depend on modeling the volume mass density and the three dimensional distant dependency of the velocity dispersion, the more recent measurements rely on the orbital analysis of the innermost stars (predominantly S2). Using the closest stars possible, the mass estimate may now be expected to settle around a value of 4.0 million solar masses with an uncertainty of about 0.2 million solar masses (Boehle et al, 2016; Parsa et al, 2017). As mass and distance are determined simultaneously from stellar orbits, future improvements of the uncertainties must involve further improvements of the positional and spectroscopic measurement accuracy.

\subsection{Size}

Knowing the distance and the mass we can now discuss the observed and estimated sizes of the black hole at the Galactic Center. Knowing the size then allows us then to calculate the mass density and compare it to mass densities expected for 


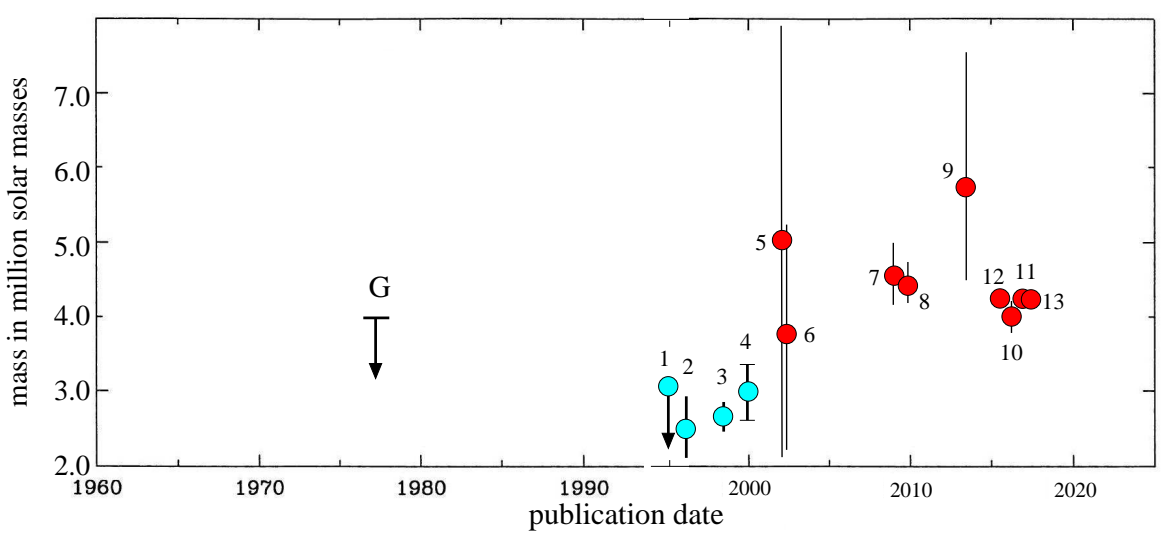

Fig. 6 Mass of SgrA* at the Galactic Center: The enclosed mass estimate derived from minispiral gas measurements by Wollman et al (1977) is marked with a bold face "G". Mass estimates derived from stellar radial velocity and proper motion measurements are shown by turquoise filled circles and labeled: 1: Krabbe et al (1995)); 2: Eckart and Genzel (1996); 3: Ghez et al (1998); 4: Genzel et al (2000). Mass estimates derived from orbital curvatures and stellar orbits are shown by red filled circles and labeled: 5: Eckart et al (2002); 6: Schödel et al (2002); Horrobin et al (2004); Eisenhauer et al (2003);7: Ghez et al (2008); 8: Gillessen et al (2009a); 9: Do et al (2013); 10:Boehle et al (2016); 11:Parsa et al (2017).

a SMBH of the given mass. Hence, we can verify how compact the massive object at the center is. As a size we may refer to a measurable diameter of the smallest possible region that contains the mass of the black hole. For a black hole the event horizon qualifies for such a designation although it can be regarded a unobservable by definition (see sections 1 and 2). But one can get very close to it (see sections 4.9 and 8). The core radius of hypothetical dense clusters (Plummer models 16 with an exponent of $\alpha=5$ ) constrained by the peribothron distance of the S2 orbit that could hypothetically still explain the total mass concentration would have a radius close to $0.22 \mathrm{mpc}$. Such a cluster would have a very short live time of less than $10^{5} \mathrm{yr}$. The radius of a neutrino ball composed of degenerate $17 \mathrm{keV}$ neutrinos, for example, is difficult to reconcile; as summarized in section 5.1 on alternative models to the black hole.

In Fig. 77, we show a comparison of size scales at the Galactic Center near Sgr A*. Shen et al (2005) report on a radio image of Sgr A* at a wavelength of $3.5 \mathrm{~mm}$, demonstrating that its size is $1 \mathrm{AU}$ (or $4.9 \mu \mathrm{pc}$ ). When combined with the lower limit on its mass (Reid et al (2003b), see also Reid et al (2003a)), the lower limit on the mass density is $6.5 \times 10^{21} \mathrm{M}_{\odot} \mathrm{pc}^{-3}$, which provides the most stringent evidence to date that Sgr $\mathrm{A}^{*}$ is a $\mathrm{SMBH}$. The black hole mass density for 4 million solar masses in a sphere of the corresponding Schwarzschild radius is $1.63 \times 10^{25}$ $\mathrm{M}_{\odot} \mathrm{pc}^{-3}$.

1.3mm VLBI source structure smaller than the expected apparent size of the SgrA* black hole event horizon has been observed by Doeleman et al (2008), suggesting that the bulk of SgrA* emission may not be centered on the black hole, but arises in the surrounding accretion flow. As shown in Fig.e,f this can be explained either as a compact jet foot-point or as the Doppler enhanced, approaching side of an - at least temporary luminous - accretion flow or disk. The intrinsic size of Sgr $\mathrm{A}^{*}$ is equal to $37_{-10}^{+16} \mu \mathrm{as}(\sim 1.5 \mu \mathrm{pc}$; see Fig. 7$)$. The corresponding $3 \sigma$ upper limit of the source size at $1.3 \mathrm{~mm}$, combined with a lower limit to the mass

\footnotetext{
16 A Plummer 3-dimensional density profile with the Plummer radius $r_{0}$ is given by $\rho=\rho_{0}(1+$ $\left.\frac{r}{r_{0}}\right)^{-\alpha}$. Functions of this form are often used as models to describe the density profile of dense stellar clusters.
} 

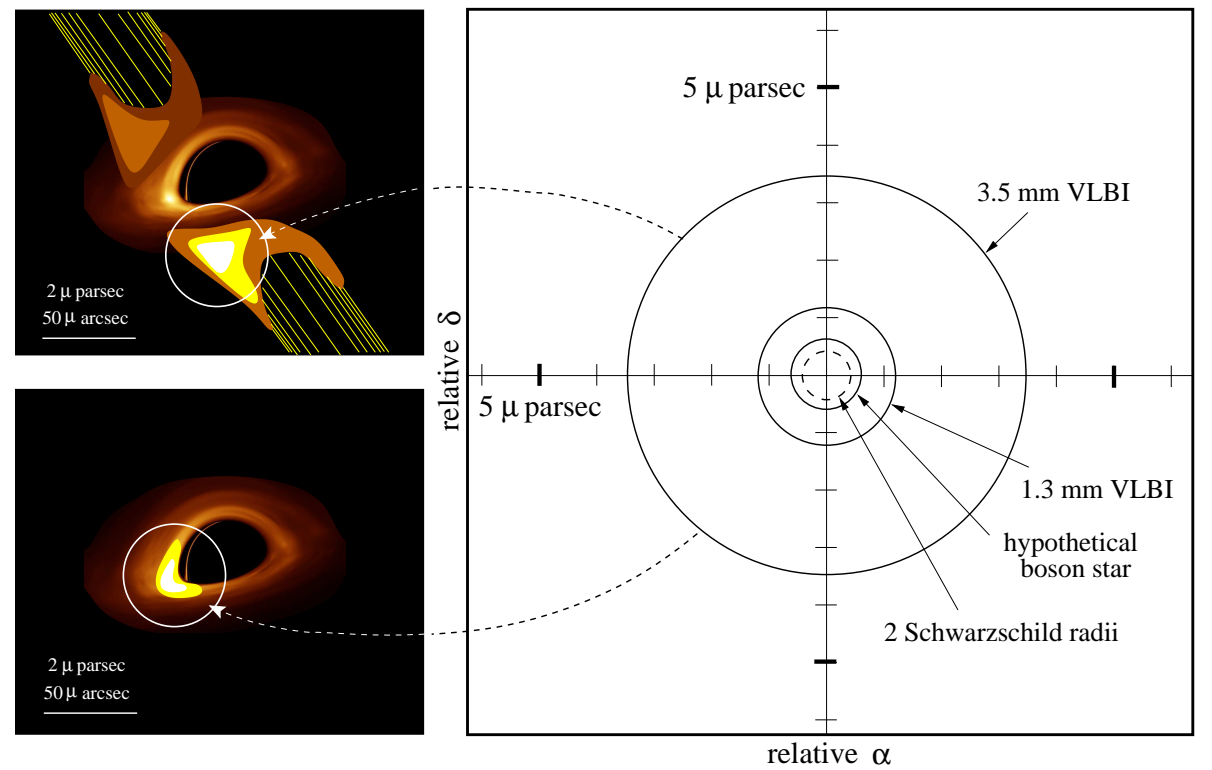

Fig. 7 A comparison of size scales at the Galactic Center near Sgr A*. We show results from theoretical predictions. On the right we depict different size estimates and scales. In this section of the image we assume that the resolution elements are centered on the position of the SMBH. However, on the left we are centered observationally on the peaks of the brightness distributions. The white circle highlights the brightest structure in the image and indicates a $3.5 \mathrm{~mm}$ (full width at half maximum) VLBI beam. To the upper left we show the the expected image in the case of a jet foot-point case and to the lower left the accretion disk case. The VLBI beams have been calculate assuming a $4500 \mathrm{~km}$ baseline. For a rotating helical jet the jet brightness profiles are highly asymmetric and due to boosting and light bending effects for medium to low inclinations the receding counter-jet will dominate the jet section pointed towards the observer, here the lower right site of the jet foot-point panel; see Dexter et al (2012b). Approximate size scales for the $\mathrm{SgrA}^{*}$ case are given in the lower left hand side of each panel.

of Sgr A* of $4 \times 10^{5} \mathrm{M}_{\odot}($ Reid et al, 2003b), yields a lower limit for the mass density of $9.3 \times 10^{22} \mathrm{M}_{\odot} \mathrm{pc}^{-3}$. Fish et al (2011); Doeleman et al (2009) Doeleman et al (2009) show that source components orbiting the SMBH can be detected even with non-imaging EHT data sets, and Fish et al (2011, 2016) confirm strong evidence for time-variable changes in SgrA* on scales of a few Schwarzschild radii using EHT observations. With these refined analysis tools and successful pre-cursor observations, the prospects for directly probing the event horizon with the EHT are excellent, and present exciting opportunities to constrain physics and dynamics at the black hole boundary, as well as potential tests of GR, in conjunction with NIR observations (Psaltis et al, 2016a). Also in Fig. 7 we show for comparison a typical size of a few Schwarzschild radii in the hypothetical case of a boson ball as summarized in section 5.1 on alternative models to the black hole. 
4.4 Spin, Inclination, and frame-dragging

The spin 17 is one of the fundamental quantities that characterize the black hole. In combination with the inclination its value is a means to probe the observability of predicted effects (see sections 4.8 and 4.10). Eckart et al (2006b) report polarization measurements of the variable near-infrared emission of $\mathrm{SgrA}^{*}$ and investigate the physical processes responsible for the variable emission from SgrA*. The authors find that the variable NIR emission of SgrA* shows highly polarized flux density excursions supporting that the NIR emission is non-thermal and could come from a jet or temporary disk model. In comparison to an orbiting spot model they find that the variability is consistent with a spin parameter of $\mathrm{a}=0.5$ and appreciably large inclinations (see also Shcherbakov et al, 2012; Vincent et al, 2011b; Broderick and Loeb, 2006; Trippe et al, 2007). Partially ordered variable magnetic fields are also supported by recent $1.3 \mathrm{~mm}$ VLBI measurements with the EHT (Johnson et al, 2015). Mever et al (2006) model their NIR polarimetry data successfully with a combined spot/ring model. They conclude that the inclination $i$ of the spot orbit must be larger than $20^{\circ}$ and the dimensionless spin parameter of the black hole is derived to be a $>0.5$. Mever et al (2007) find that their observations support an approximately constant mean polarization angle of $60^{\circ} \pm 20^{\circ}$ between 2004-2006.

The spacetime frame-dragging (Lense-Thirring) effects near a rotating compact object can be of conceptual and also considerable astrophysical importance for the models of black holes surrounded by orbiting material, such as an accretion ring or a disk-like structure (Barbour and Pfister, 1995). Naturally, these effects are the strongest near a rotating black hole, where the ergospheric region develops. For compact objects like stars probing the gravitational field of the SMBH, the orbital period $P$ will depend on the black hole mass $M$ and the long axis $a$ of the orbital ellipse and the relativistic effect of the high mass concentration will result in an angular advance of the peribothron (periapse for black holes) angle $\omega$,

$$
\begin{aligned}
& P \propto a^{1.5} M^{-0.5} \\
& \omega \propto a^{-1} M\left(1-e^{2}\right)^{-1} .
\end{aligned}
$$

In addition, precession of the orbits will be affected by the Lense-Thirring effect and the quadrupole moment of the black hole. The characteristic timescales for these three effects that dominate are $t_{M}$ (orbital precession), $t_{J}$ (Lense-Thirring precession), and $t_{Q}$ (quadrupole precession). They depend on successively increasing powers of the orbital long half axis and eccentricity factor $\left(1-e^{2}\right)$ and a decreasing power of the black hole mass (Merritt, 2010; Merritt et al, 2010; Psaltis et al, $2016 \mathrm{~b}, \mathrm{a})$.

$$
\begin{aligned}
t_{M} & \propto a^{2.5} M^{-1.5}\left(1-e^{2}\right) \\
t_{J} & \propto a^{3.0} M^{-2.0}\left(1-e^{2}\right)^{1.5} \\
t_{Q} & \propto a^{3.5} M^{-2.5}\left(1-e^{2}\right)^{3}
\end{aligned}
$$

This shows that the objects of very eccentric orbits (e approaching 1$)$ close to the black hole mass are preferred and the higher order quantities like the precession due to Lense-Thirring and quadrupole moment become successively smaller. Specific precession frequencies induced by the frame dragging could for instance be

\footnotetext{
17 For black holes the spin can be characterized by via the angular momentum parameter $a=$ $J / M c$, where $J$ is the angular momentum and $M$ the mass of the black hole. It has the dimension of a length and lies in the interval $a^{2} \leq\left(G M / c^{2}\right)^{2}$. This results into the dimensionless parameter $a^{*}=a c^{2} / G M$, which now lies between 0 and 1 . Non-rotation black holes have $a^{*}=0$ and maximally rotation black holes have $a^{*}=1$. The spin is usually determined from modeling spin dependent quantities, like light curves of orbiting hot spots or observing jets (in section 4.8) or searching for a black hole shadow (in section 4.10).
} 
detected in motion of stars. The number of stars available to prove relativistic effects within the central arcsecond (about $10^{5}$ Schwarzschild radii) can be estimated to be several hundred for a NIR brightness larger than $21^{\text {st }}$ magnitude in K-band at $2 \mu$ wavelength and about 40 stars between $18^{\text {th }}$ and $16^{\text {th }}$ magnitude in the K-band (Sabha et al, 2012; Do et al, 2009; Rubilar and Eckart, 2001; Jaroszynski, 1999). Relativistic effects are strongest close to the supermassive object. Here measurements potentially also allow us to distinguish between a SMBH and a boson star (see 5.2). If we restrict ourself to the central $1000 \mathrm{AU}$ we can estimate the number of test objects that are available to probe the relativistic effects.

We estimate the amount of stellar mass $M_{*}(a)$ surrounding SgrA* inclosed within a circular orbit of semi-major axis $a$ as

$$
\frac{M_{*}(a)}{M_{S g r A *}}=\left(\frac{M_{*}}{M_{S g r A *}}\right)\left(\frac{a}{a_{0}}\right)^{3-\gamma} .
$$

Here $\gamma$ is the exponent of the stellar number density distribution around the center. We assume that within $a_{0}=1 p c$ a total mass of $M_{*}=10^{6} \mathrm{M}_{\odot} \quad$ is contained in stars with typically one solar mass (Merritt, 2010). The results are summarized in Tab. 1. Here it becomes evident that the number of objects strongly varies with the value of $\gamma$. We have used a value of $\gamma=2$ (Merritt, 2010), close to the value of $\gamma=7 / 4$ for relaxed stellar clusters around SMBHs (Bahcall and Wolf, 1976). We also used values for flatter distributions with $\gamma \sim 1.2$ and $\gamma \sim 1.0$ found for early and later type stars within the central arcseconds around SgrA* (Buchholz et al, 2009; Schödel et al, 2007). If in Tab. 1 the number of objects within the central 1000 AU gets close to or even drops well below unit. In addition, these numbers can be looked upon as upper limits, for scattering events may help to empty the central region around the SMBH. In Tab. 1 we have also listed the expected number of stars observed in the NIR K-band within the magnitude interval of $\mathrm{K}=18$-19 (see e.g. Sabha et al, 2012). These will be suitable for NIR interferometry with GRAVITY at the VLTI (see section 6).

Table 1 Number of stellar objects within 1000 AU of SgrA*

\begin{tabular}{cccc}
\hline \hline$\gamma$ & $\mathrm{N}_{\text {stars }}$ & $\mathrm{N}_{m s P}$ & $\mathrm{~N}_{n P}$ \\
\hline 2.0 & 5000 & 5 & 0.5 \\
1.2 & 67 & 0.67 & 0.067 \\
1.0 & 24 & 0.24 & 0.024 \\
\hline 2.0 & 6 & - & - \\
1.2 & 0.08 & - & - \\
1.0 & 0.03 & - & - \\
\hline \hline
\end{tabular}

Approximate number of stars, millisecond pulsars $m s P$, and normal pulsars $n P$ with distances to SgrA* of less than 1000 AU. This corresponds to a radius of 0.125 " or $4.7 \mathrm{mpc}$. Using a value of $M_{*}=10^{6} \mathrm{M}_{\odot}$ for the central paresc we derive for different values of $\gamma$ the number of solar mass stars (second column in the top three rows) and stars with a $2 \mu \mathrm{m}$ wavelength brightness in the magnitude interval $\mathrm{K}=18-19$ (second column in the bottom three rows). Using the estimate of 100 normal and 1000 millisecond pulsars within the central parsec (Wharton et al, 2012; ; Psaltis et al, 2016b,a). we derived the corresponding values for the centra $1000 \mathrm{AU}$ in cloumns 3 and 4.

While in Tab. 1 the number of pulsars is assumed to be a constant fraction (see table caption) of stars distributed with the power-law index $\gamma$, more detailed calculations can be performed that allow different kinds of stars to have different power-law exponents. Similarly Hopman and Alexander (2006) calculated the inner radius where the stellar cusp ends in a statistical manner (low probability of detection) for different stellar components (general notation $M$ ): main-sequence stars (MS), white dwarfs (WD), neutron stars (NS), and black holes (BH). The general relation may be expressed as, 
Table 2 The inner radii of the stellar cusp for different stellar populations $M$ calculated according to Eq. 17

\begin{tabular}{ccrrrr}
\hline \hline $\begin{array}{c}\text { Stellar } M \\
\text { population } M\end{array}$ & $C_{M}$ & $\gamma_{M}$ & $r_{1, M}[\mathrm{pc}]$ & $r_{1, M}[\mathrm{AU}]$ & $r_{1, M}[\mathrm{mas}]$ \\
\hline MS & 1 & 1.4 & $2 \times 10^{-4}$ & 41 & 5 \\
WD & 0.1 & 1.4 & $7 \times 10^{-4}$ & 144 & 18 \\
NS & 0.01 & 1.5 & $20 \times 10^{-4}$ & 412 & 50 \\
BH & 0.001 & 2 & $6 \times 10^{-4}$ & 124 & 15 \\
\hline
\end{tabular}

$$
r_{1, M}=\left(C_{M} N_{h}\right)^{-1 /\left(3-\gamma_{M}\right)} r_{\mathrm{h}}
$$

where $N_{\mathrm{h}}$ is the total number of MS stars, $C_{M} N_{h}$ is the total number of stars of type $M$ within the radius of influence of the black hole $r_{\mathrm{h}}=G M_{\bullet} / \sigma^{2}\left(M_{\bullet}\right.$ is the black hole mass and $\sigma$ is the stellar velocity dispersion), and $\gamma_{M}$ is the power-law exponent for stellar type $M$.

According to the analysis of Hopman and Alexander (2006) the total number of MS stars within the radius of the gravitational influence $r_{h}=2 \mathrm{pc}$ is $N_{h}=3.4 \times 10^{6}$. Table 2 summarizes the inner radii of the cusp for different components. The range of power-law exponents Hopman and Alexander (2006) obtained in their dynamical calculation for different stellar populations (see Tab. 2) are included in the range of exponents covered in Tab. 10. The fact that for pulsars the number of objects gets close to unity or actually drops below unity within $1000 \mathrm{AU}$ is consistent with the inner cusp radius of about 400 AU for neutron stars. According to Tabs. 1 and 2. the number of objects within the central 1000 AU (inside the peribothron of S2 star) gets close to or drops below unity. This indicates that it is very unlikely that in there a star can be found and used to probe relativistic effects.

Specific precession frequencies induced by the frame dragging could also be detected in the time variable signal from $\mathrm{SgrA}^{*}$ at the Galactic Center (i.e. precession of temporary accretion disks and the corresponding modulation in the flux density), but also from a number of (more distant to us) active galactic nuclei (see also $\mathrm{Wu}$ et al, 2016). The observation of these signatures could provide us with independent evidence for the presence of a rotating black hole in these objects. Solutions of Einstein equations for self-gravitating disks or rings with or without a central black hole have been found in the past (see e.g. Bardeen and Wagoner, 1971; Will, 1974; Neugebauer and Meinel, 1993). Self-gravitating disks around relativistic spheroidal configurations have also been considered - however, those would be massive. They are important for extragalactic SMBHs, but they are probably not of relevance in the case of the Galactic Center. Both the angular momentum of the black hole and the angular momentum of the surrounding disk contribute to the total dragging. If the black hole rotates slowly and the disk has sufficient mass, the maximum of the dragging effect is located close to the center of the disk rather than at the horizon (Karas et al, 2004). A possible observational consequence is that the light rays near a self-gravitating disk are significantly distorted, which results in the change of the spectrum compared to the case when the disk self-gravity is ignored. Another consequence is that trajectories of massive bodies near the disk are attracted to it. Similar to the effects on massive particles, the frame-dragging effects can be revealed in the shape of magnetic lines of force that are also affected by rotation of the black hole, twisting the magnetic structures in its close vicinity (Karas et al, 2012) In the case of the Galactic Center the observable frame-dragging effects near $\mathrm{SgrA}^{*}$ are most likely limited to the stellar motion - if stars close enough to SgrA* can be found - and characteristic quasi-periodic modulations of the light curves. 


\subsection{Orientation}

The orientation angle $\theta$ of the inclined spin axis on the sky may be linked to the directions under which jet or wind interactions with the surrounding interstellar medium are claimed (see right side of Fig. 4, section 4.8 and discussion by Shahzamanian et al, 2015b; Li et al, 2013; Yusef-Zadeh et al, 2012a; Eckart et al, 2006b a; Morris et al, 2004). The theoretical study of outflows from RIAF was performed by Yuan et al (2012), Based on NIR polarimetry data taken over a time range of 8 years Shahzamanian et al (2015a) find typical polarization degrees that are on the order of $20 \%-10 \%$ and a preferred polarization angle of $13^{\circ} \pm 15^{\circ}$. The emission is most likely due to optically thin synchrotron radiation, and the preferred polarization angle is very likely coupled to the intrinsic orientation of the Sgr A* system, i.e. a disk or jet/wind scenario associated with the SMBH. Shahzamanian et al (2015a) conclude that if the polarization properties are linked to structural features, then the data imply a stable geometry and a stable accretion process for the Sgr $\mathrm{A}^{*}$ system. The polarization position angle taken as a measure of orientation of the $\operatorname{SgrA}^{*}$ system on the sky matches quiet well with the relative location of the mini-cavity (at an angle of about $\theta=193^{\circ}$, i.e., $\theta=13^{\circ}+180^{\circ}$ ) that may be due to the interaction of a nuclear wind from Sgr $\mathrm{A}^{*}$ with the mini-spiral material (see also Rozanska et al, 2017). The cometary tails of sources X3 and X7 reported by Mužić et al (2010) lie within this range of angles and can be taken as a strong observational support for the presence of a fast wind from $\operatorname{Sgr} \mathrm{A}^{*}$.

Modeling the polarized light curves with a relativistic disk or hot-spot system results in model dependent information on the orientation of such a system on the sky. Using near-infrared polarimetric observations and modeling the terms of an orbiting spot, Meyer et al (2007) constrain the three dimensional orientation of the Sgr A* system. They find that the position angle of the equatorial plane normal is in the range $60^{\circ}-108^{\circ}$ (east of north) in combination with the large inclination angle. This is in agreement with the orientation for emission from disk components with magnetic field lines perpendicular to the disk as described by Zamaninasab et al (2010). In Zamaninasab et al (2010), the authors also find significant evidence from emission of matter orbiting a SMBH.

A range of orientation angles of the $\mathrm{SgrA}^{*}$ system on the sky may be linked to jet or wind phenomena that are observed within the central stellar cluster. A detailed summary and discussion of these phenomena is given by Shahzamanian et al (2015a). Without a clear kinematic evidence for a jet or wind and its connection to SgrA*, no firm conclusions can be drawn on how the system is oriented in the sky and how the polarization and modeling information can be interpreted in this context. Psaltis et al (2015) and Psaltis et al (2016a) discuss observations with the Event Horizon Telescope at $1.3 \mathrm{~mm}$ which have revealed a size of the emitting region that is smaller than the size of the black-hole shadow. They argue that this can be reconciled with the spectral properties of the source, if the accretion flow is seen at a relatively high inclination $\left(50^{\circ}-60^{\circ}\right)$. Psaltis et al (2015) and and Psaltis et al (2016a) claim that such an inclination makes the angular momentum of the flow, and perhaps of the black hole, nearly aligned with the angular momenta of the orbits of mass-losing stars that lie within about 3" from the black hole and are the main source for the putative accretion stream onto SgrA* (see also appendix by Mužić et al, 2007).

\subsection{Spectrum}

SgrA* shows variable emission from the radio to the X-ray domain. The overall spectrum of SgrA* may be used to derive general properties of the immediate phys- 

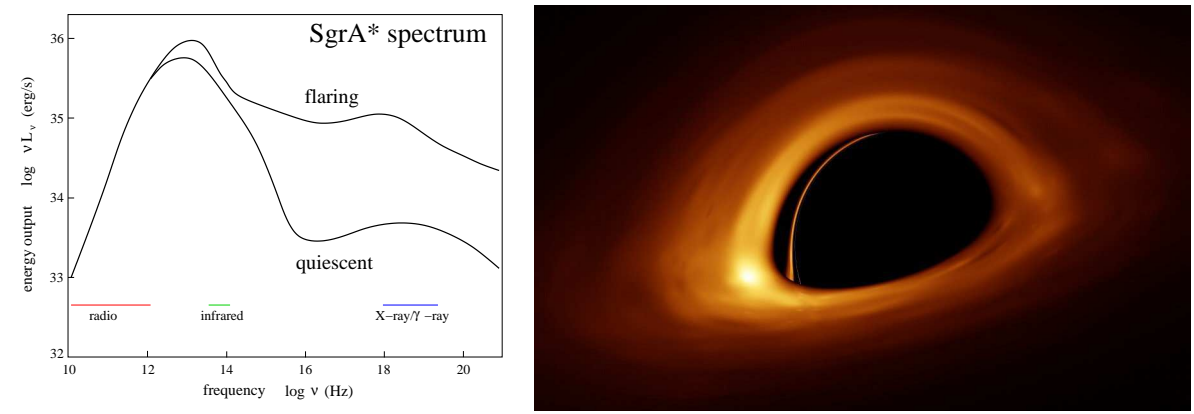

Fig. 8 Left: Sketch of the broad band electromagnetic spectrum of Sgr A*. Data can be obtained in the accessible and useful radio, infrared and X-ray $/ \gamma$-ray windows. The black curves represent a model spectrum during quiescence and during a bright flare of $\operatorname{Sgr} \mathrm{A}^{*}$. The model spectrum matches the observed data well and predicts what is expected in the non-accessible regions of the spectrum. Right: Model image of the shadow of the black hole (see Bursa et al (2007) more details on the calculations). Relativistic effects brighten the right left side and dim the right side of the temporary accretion disk surrounding the black hole at the center. For suitable inclinations of the system, relativistic effects (e.g. light bending and abberation) produce a dark region close to the position of the black hole and covering about a fourth of the shown image section. For a non-rotation black hole of $\sim 4.3 \pm 0.3 \times 10^{6} \mathrm{M}_{\odot}$, the image has a size of about $9.4 \times 15.4$ Schwarzschild radii corresponding to an angular size of about $160 \mu \mathrm{as} \times 100 \mu \mathrm{as}$.

ical surrounding of SgrA*. As in the case of stellar accreting black holes the spectral properties of this region for accreting SMBHs are intimately linked to the accretion state and the black hole location in a fundamental plane expressing that state (e.g. Markoff et al, 2011; Markoff, 2005; Vasudevan and Fabian, 2007). On the left side of Fig 8 we show a sketch of a spectrum of $\mathrm{SgrA}^{*}$ in quiescent and in a flaring state from the radio to the X-ray/ $\gamma$-ray domain. Baganoff et al (2003) show that the Xray emission at the position of Sgr $\mathrm{A}^{*}$ is extended, with an intrinsic size of $\sim 1.4$ " (FWHM), consistent with the Bondi accretion radius for a several million solar mass black hole. However, SgrA* is much fainter than expected at all wavelengths, especially in X-rays. Baganoff et al (2001) report the first discovery of rapid X-ray flaring from $\mathrm{SgrA}^{*}$ using the Chandra observatory in the (0.5-7 keV)-band implying that this part of the X-ray emission is due to accretion of gas onto a SMBH.

Eckart et al (2012b) report on new simultaneous observations and modeling of the millimeter, near-infrared, and X-ray flare emission of the source SgrA*. The authors study how and if the concept of the adiabatic synchrotron source expansion can be applied to the variable emission of SgrA*. Source component sizes are typically around one Schwarzschild radius and the peak or the flare emitting spectrum lies around 300-400 GHz or just short of $1 \mathrm{THz}$. The bulk of the emission can be explained through Synchrotron-Self-Compton (SSC) scattering from these synchrotron sources into the X-ray domain (see also Yusef-Zadeh et al, 2011, 2012b).

Modeling of the light curves shows that the sub-mm follows the NIR emission with a delay of about three-quarters of an hour with an expansion velocity around $10^{-2}$ of the speed of light. In the radio flare the variability often shows signs of adiabatically expansion of these synchrotron source components.

Assuming that the bulk of the emission from SgrA* originates from accretion of matter provided by streamers and strong stellar winds in the central stellar cluster, SgrA* provides an outstanding case for a radiatively inefficient accretion flow (RIAF), a favorite model for the accretion of matter onto SgrA* with accretion efficiencies well below the standard thin-disk accretion flow (Quataert, 2003; Yuan and Narayan, 2014). SgrA* radiates at about $10^{-9}$ times the Eddington luminosity which is the maximum that can be achieved for spherical accretion onto a massive black hole. Hence, SgrA* is the weakest black hole accessible to de- 
tailed investigations (Markoff et al, 2007). While nearly spherical accretion occurs if the angular momentum content of accreted material is negligible, in another limit one ignores radial transport of the material and considers an axially symmetric toroidal configuration that preserves permanent rotation about the symmetry axis. The treatment of self-gravitating discs was originally introduced in Ostriker's Newtonian equilibria of uniformly rotating, polytropic, slender rings Ostriker (1964). Later, self-gravitating configurations with realistic equation of state and opacity were constructed and the basic formalism for self-gravitating black hole accretion discs was given in GR (Bardeen, 1973). Self-gravity has global consequences on the disk shape: it influences the location of its inner and outer edges, as well as the disk geometrical thickness and their vertical structure.

Radiatively inefficient accretion flows (RIAFs) are believed to power SMBHs in the underluminous cores of galaxies (Mościbrodzka et al, 2014).

Such black holes can typically be associated with compact radio sources with flat or inverted $\mathrm{cm}-/ \mathrm{mm}$-radio spectrum as it is the case for SgrA*. Using threedimensional general relativistic magneto-hydrodynamics (MHD) accretion flow simulations, Mościbrodzka et al (2014) can show that the radio to X-ray properties of SgrA* can very well be explained though a RIAF model under the conditions found at the center of the Milky Way. In this case the X-ray emission is very sensitive to the electron heating mechanism in the immediate surroundings of the SMBH.

\subsection{Variability}

Given that the potential accretion of stellar winds and mini-spiral gas may be a turbulent process, one can expect that this will result in variable accretion and hence variable flux densities from the SMBH. Alternatively the magnetic field strength and configuration may be affected by the interactions with the interstellar medium which would also result in strong variability. Falcke and Markoff (2000) present a summary of the wavelength dependent variability information for $\mathrm{SgrA}^{*}$ from the radio to the X-ray domain. The authors found that synchrotron and synchrotron SSC provides an excellent fit to the entire broad band data. The SSC process also efficiently explains the strong variability of $\mathrm{SgrA}^{*}$ seen at X-ray bands. If $\mathrm{SgrA}^{*}$ has a jet, then it must be short or of very low surface brightness such that it naturally satisfies the resolution and sensitivity limits set by the interstellar scatter and VLBI experiments.

Witzel et al (2012a) characterize the statistical properties of the NIR variability of Sgr A*. They find that it is consistent with a single-state process, hence, forming a power-law distribution of the NIR flux density measurements. The authors also show that it is difficult with the current total power continuum data to decide on the existence of e.g. a quasi-periodic signal that is significant above the expected from a red-noise random process. Neilsen et al (2015a) present a statistical analysis of the X-ray flux measurements of Sgr A* obtained with the Chandra observatory describing $\mathrm{SgrA}^{*}$ as a composite of a stationary source and a variable component as expected for the inner section of an accretion flow. Neilsen et al (2015a) find that the variable component contributes about $10 \%$ of the overall quiescent flux. Barrière et al (2014) report the detection of SgrA* at energies of up to $79 \mathrm{keV}$ using the NuSTAR observatory. SgrA* continues to show strong variability at these energies as well and there is apparently not sign for a cutoff in variability towards high energies. Variable emission at high energies must result from inverse Compton scattering or from highly efficient continuous particle acceleration and high magnetic field strengths as the synchrotron cooling time is of the order of $1 \mathrm{~s}$. Barrière et al (2014) highlight that with the variability time scales and the total en- 
ergy emitted, the model-dependent location of the flares must be as close as about 10 Schwarzschild radii from the black hole.

4.8 Does Sgr $A^{*}$ have a jet or an outflow?

Many (if not all) SMBHs in extragalactic low luminosity active galactic nuclei are associated with jets that are most likely launched from their accretion disks. Hence, the assumption is that $\mathrm{SgrA}^{*}$, although it is not an active galactic nucleus but is located at the lower end of the luminosity distribution (e.g. Eckart et al, 2012a; Contini, 2011), may have a jet or at least a strong wind, too. Often the "Fermi bubbles" are quoted as potential observational signatures of strong outflow from hot accretion flow in the Galactic Center (Dobler et al, 2010; Su et al, 2010; Mou et al, 2014, 2015). These bubbles extend to about $50^{\circ}$ above and below the Galactic plane, and exhibit a width of about $40^{\circ}$ in Galactic longitude. They are thought to be due to the interaction between the interstellar medium (ISM) and winds that have been launched from the hot accretion flow from SgrA* and the central starforming regions.

Proving the existence of a jet or strong wind would be very supportive for the existence of a SMBH. Although observationally indicated (Li et al, 2013; Yusef-Zadeh et al, 2012a), a jet or wind from SgrA* is expected but the evidences for it are not very clear (see discussion by Shahzamanian et al, 2015b; Eckart et al, 2006b, a; ; Morris et al, 2004). The theoretical study of outflows from RIAF was performed by Yuan et al (2012), who show for the first time the existence of a strong outflow launched from the accretion flow. (Blandford and Begelman (1999) only assume the existence of outflow.) Yuan et al (2012) show that the inward decrease in the accretion rate which depends on the radius as $\frac{d}{d r} M_{a c c} \propto r^{s}$ (where $s \sim 0.5-1.0$ ) is explained by the significant mass loss via wind. MHD calculations predict a central plane and outflow region for the relativistic electron density distribution (e.g. Dexter and Fragile, 2013; Dexter et al, 2010; Mościbrodzka and Falcke, 2013). However, with decreasing radio wavelength the angular resolution of VLBI measurements is decreasing and interstellar scattering is getting more severe as well (Markoff et al, 2007; Britzen et al, 2015).

Hence, this outflow region is difficult to be measured. At mm-wavelengths the effects of interstellar scattering can be overcome and the source intrinsic structure of $\mathrm{SgrA}^{*}$ can be investigated. However, steep spectra and low surface brightness makes it a challenge to search for a wind or jet from SgrA* at high frequencies.

\subsection{Event Horizon or surface?}

In classical (non-quantum) GR, a black-hole horizon is defined as a null hypersurface formed by light rays that are just on the verge between escaping to infinity and being trapped by the strong gravity. As a consequence, the horizon is of rather ill determined nature in the sense that one has to know the entire history of light rays and the null structure of the spacetime to be able to determine the existence and the actual location of the horizon (see Israel, 1987)). Could SgrA* be supermassive but have no event horizon? If such a possibility can be ruled out, this would speak in favor of it being a black hole. Ruling out such a possibility would speak in favor of a black hole. The presence of a jet implies a high accretion rate either onto an accretion disk, a hard surface, or an event horizen. The presence of a jet origination from the vicinity of SgrA* would imply an at least temporally present accretion disk that may maintain magnetic flux required for the jet confinement and launching near the black hole. Regardless of the jet launching mechanism, a minimum mass accretion 
rate would be necessary to power this scenario. This has been discussed in detail for the M87 jet by Broderick et al (2015). In the case of SgrA*, such an accretion rate is provided by the surrounding mass-losing young stars. Accreting this material onto a hard surface instead through an event horizon would result in considerable thermal near-infrared emission from the surface due to shocks (e.g. Narayan et al, 1998; Verozub, 2006). The fact that $\mathrm{SgrA}^{*}$ is sub-luminous even with respect to the Eddington luminosity, which results from the highest rate at which matter can be spherically accreted onto black holes, implies the presence of a black-hole horizon rather than a hard surface.

It appears to be virtually impossible to unambiguously prove the presence of black-hole horizon by observing the electromagnetic signal from its presumed vicinity to such a horizon. Here, the absence of a radiation signal does not necessarily prove the absence of the surface of the body and the existence of the horizon (see the critical discussion of tentative proofs, like general properties of accretion flows, dimness, absence of X-ray bursts, in Abramowicz et al, 2002). However, a very suggestive and almost convincing evidence of the strong gravity associated with stellar or supermassive black hole would be a detection of light encircling the black hole along a photon orbit and thus leading to multiple images that should occur with a precisely defined time delay. As can be seen by the communication between the Czech engineer Rudi Mandl and Albert Einstein (Renn and Sauer, 2005; Einstein, 1936) the concept of light bending in the context of high mass concentrations has been discussed at a very early stage. Bursa et al (2007) conclude that if the delay in arrival time between the "direct" and "looped" photons can indeed be revealed in the light curves, this would provide a direct evidence for the existence of circular photon orbits. In this way, it will be possible to demonstrate the validity of an important prediction of GR in the regime of strong gravitational fields.

4.10 Black Hole Shadow: proving GR and observing the photon sphere at the event horizon

Very long baseline radio interferometry in the $\mathrm{mm}$-wavelength domain promises to image the immediate vicinity of the $\mathrm{SgrA}^{*} \mathrm{SMBH}$ in the foreseeable future. It is expected that the immediate surroundings of the $\mathrm{SgrA}^{*} \mathrm{SMBH}$ is lit up by accretion processes. Due to the bending of light by the black hole there is the possibility that a dark region might appear in this region. Falcke et al (2000) have first predicted the size of this so-called shadow for Sgr A* of about $30 \mu \mathrm{as}$. (see right side of Fig (8). The exact size, location and observability of the shadow will depend on the inclination and spin of the black hole. However, it is within reach of current or upcoming VLB interferometers in the mm-wavelength domain. These measurements must take into account that the imaging at least at wavelengths less than $1.3 \mathrm{~mm}$ is hampered by interstellar scattering which is also responsible for part of the flux and structural variability. Gwinn et al (2014) have detected substructure within the smooth scattering disk of the $\mathrm{SgrA}^{*} \mathrm{~mm}-\mathrm{VLBI}$ radio image as a consequence of refraction in the interstellar medium. Rauch et al (2016) have detected a secondary radio off-core feature associated with flux density variations at $\mathrm{mm}$ - and NIR-wavelengths. Fish et al (2014) and Lu et al (2016) present a procedure to mitigate the effects of interstellar scattering. They show that a black hole shadow and a photon ring (if both are indeed present) can clearly be detected if one makes use of observations over multiple days and creates an image of the average quiescent emission. Broderick et al (2014) and others claim that the EHT-observations provide the novel opportunity to test the applicability of the Kerr metric to astrophysical black holes. Simulations by several groups have been prepared for this black hole shadow (e.g. Ricarte and Dexter, 2015; Broderick et al, 2014; Falcke et al, 2000). 
Broderick et al (2014) present the first simulated images of a radiatively inefficient accretion flow (RIAF) around SgrA* employing a quasi-Kerr metric that contains an independent quadrupole moment in addition to the mass and spin that fully characterize a black hole in GR. They show that these images differ significantly from the images of an RIAF around a Kerr black hole with the same spin and demonstrate the feasibility of testing the no-hair theorem by constraining the quadrupole deviation from the Kerr metric with existing EHT data. However, they claim that at present, the limits on potential modifications of the Kerr metric remain weak.

\subsection{Pulsars at the Galactic Center}

A very efficient way to map out space-time in the vicinity of the central supermassive black hole is to find and track pulsars orbiting SgrA*. The central black hole spin and quadrupole moment could be measured with a very high precision by the pulsars that are only close enough to the black hole. Tracking the orbital motion of pulsars would allow us to test different theories of gravity (Psaltis, 2012). Although it is generally agreed on that there must be a large number of stellar remnants at the center of the stellar cluster, i.e. in the immediate vicinity of the black bole, it is not clear how large the number of detectable pulsars in that region will actually be. These number will depend on the star formation history in the overall region and on the efficiency of the dynamical processes that let remnants gather in the very center. Dexter and O'Leary (2014) explain the "missing pulsar problem" by an intrinsic lack of ordinary pulsars. On the other hand (although the statistics is still poor), the discovery of a single magnetar within 0.1 pc may imply an intrinsic overproduction of magnetars close to the Galactic Center since the occurrence of magnetars in the standard Galactic stellar population is rare in comparison with normal pulsars. The overproduction of magnetars could take place due to either strongly magnetized progenitors or the top-heavy initial mass function (massive progenitors). Magnetars are short-lived in comparison with ordinary pulsars, which results in low likelihood of pulsar detections (Dexter and O'Leary (2014) compare sensitivity limits of past surveys with luminosities of known pulsars in their Fig. 2).

Estimates of the number of solar type stars, normal and millisecond pulsars are listed in Tab. 1. These numbers imply that the likelihood of finding even a single pulsar in a region in which the gravitational field of the central SMBH can be investigated is extremely small. However, the magnetar PSR J1745-2900 resulted in the discovery of radio pulses using the Effelsberg telescope. This discovery demonstrates that these objects are present in the central stellar cluster and that highlights the great value and the efforts that are being undertaken to find pulsars close to SgrA* (Spitler et al, 2014; Mori et al, 2013; Eatough et al, 2013). Alternatively, instead of looking for radio pulses it was proposed that several neutron stars could be potentially detected via bow shocks due to the interaction of supersonic magnetized neutron stars with ionized gas in the central parsec (Zajacek et al, 2015; Giannios and Lorimer, 2016). Psaltis et al (2016a) show that the results obtained from stars and pulsars can be ideally combined with those of shape and size of the shadow of the black hole promising a high accuracy test for the gravitational no-hair theorem.

\subsection{Any chance for charge?}

As stationary black holes are characterized by the three quantities mass, angular momentum and charge, one may raise the question if there can be a significant electrical charge associated with the SMBH at the center of the Milky Way. Although 
GR allows for a black hole to acquire electric charge, it is thought that astrophysical black holes are electrically neutral to very high precision. This is because of processes of selective charge accretion from the surrounding plasma. However, it is important to note that the surrounding material may acquire some non-zero net charge density, Slaný et al (2013), and so the interplay of electrically charged particles and magnetic fields in the strong gravitational field of black holes appears to be relevant. For example, irradiation of dust particles leads to a positive net electrical charge by photoionization. On the other hand, plasma electron and ion currents are continuously entering the grain surface, so the sign and magnitude of the equilibrium charge depend on the total currents that are absorbed and emitted from the grain surface. This is a complex process that depends on many parameters, and the resulting message is that electrostatic charge is among the essential parameters that control the dynamics of dust grains embedded in the surrounding plasma. However, in the Galactic Center this effect may be diminished greatly. The existence of smaller dust grains at the center is probably limited due to intense UV and X-ray radiation field and they plausibly completely vanish on the scale of tens of gravitational radii in the hot accretion flow. On the other hand, the continuous formation of dust in stellar winds, in particular of AGB stars (Yusef-Zadeh et al, 2017), as well as the speculative asteroid or planet infall towards Sgr A* (Zubovas et al, 2012) could in principle replenish the dust content even very close to the black hole.

Black holes without angular momentum are described by the Reissner-Nordström metric, those with angular momentum and electric charge are described by a KerrNewman metric. As the electrical force field is about 40 magnitudes larger than the gravitational field, it is thought to be unlikely that significant netto charges can be accumulated with black holes. There is the possibility that the black hole shadow disappears if an appreciable charge is accumulated (Zakharov, 2014). However, the shadow of a black hole is not a very clean observable, and inclinations and accretion phenomena may cause the disappearance (or washiness) of a shadow as well. In 2013, Tsupko \& Bisnovatyi-Kogan gave a first detailed analysis of how the images from the surroundings of black holes change in the presence of plasma (Tsupko and Bisnovatyi-Kogan, 2013). This may imply that suitable observing frequencies may lie even above $230 \mathrm{GHz}$ (Falcke et al, 2000) to avoid that the images are severly washed out.

\subsection{Gravitational wave signals from inspiraling objects}

The merging event between SMBHs among each other and with other compact objects like will produce specific ringing signals in emitted gravitational waves (Löckmann and Baumgardt, 2008; Aasi et al, 2013). In a strict sense the word "ringing" is used to describe the ring-down of the new SMBH formed in the aftermath of a SMBH binary merger. For simplicity we use it here for the gravitational wave signal we receive from inspiraling masses in general. Comparison of these signals to theoretically predicted signals is a direct indication for the presence of a SMBH. These signals may be the only radiation coming from the immediate vicinity of the black hole (see section 2.2). Extreme Mass Ratio Inspirals (EMRIs; see Amaro-Seoane et al, 2007, for a review) have also been identified as effective probes for the Kerr metric of GR by the eLISA group (Danzmann, 2015). However, the merging rates are very small and a high detection rate can only be achieved by including a large local cosmological volume. The recent measurement of a theoretically expected chirped gravitational wave signal using the Laser Interferometer Gravitational-Wave Observatory (LIGO) demonstrated the existence of binary stellar-mass black hole systems and the possibility to directly detect gravitational waves from a binary black hole merger (Abbott et al, 2016). It was reported that at a distance of about $410 \mathrm{Mpc}$ in the 
system GW 150914 two very compact objects of about $36 \mathrm{M}_{\odot}$ and $29 \mathrm{M}_{\odot}$ merged. These objects were probably black holes; however, exotic alternatives still need to be ruled out (see sections 5] and 3.1. Cardoso et al (2016), as well as a comment in The LIGO Scientific Collaboration and The Virgo Collaboration (2016)). For most of the alternative theories, predictions for the specific shape of the ringing signal still need to be performed, although at a higher frequency, these measurements validate the method proposed to search for supermassive binary black holes and support the possibility of detection gravitational wave ringing from such systems. If there are IMBHs in the central stellar cluster (e.g. the case of IRS13E Maillard et al, 2004; Schödel et al, 2005; Fritz et al, 2010), their interaction with SgrA* may produce a sufficiently strong signal if a merger event occurs. However, the likelihood for this is rather low (Aasi et al, 2014).

Expected gravitational wave events from the Galactic center are supposed to originate in the inspiral of bodies with extreme ratio of masses - supermassive black hole and a stellar remnant of a few solar masses (EMRI; see above). Extreme mass ratio means that a merging body effectively acts as a test particle in the spacetime of the supermassive black hole. Before the final plunge, it is possible to infer the properties of the spacetime from the observed waveform, since it reflects the peribothron as well as Lense-Thirring precession for a Kerr black hole. White dwarfs, neutron stars and stellar black holes are not subject to tidal disruption. Merging with a SMBH can hence result in a detectable amount of gravitational wave luminosity (some $10^{40} \mathrm{erg} \mathrm{s}^{-1}$ ) over several days (see equation 23 in Appendix (D).

The detection of an EMRI event associated with the Galactic center black hole would enable to precisely measure the mass and the spin of the black hole with an independent measurement. The waveform could also reveal deviations from the Kerr metric as well as a possible different character of a central compact object (e.g. a boson star). An exemplary waveform for an EMRI event for the ratio $m_{\star} / M_{\bullet}=10^{-4}$ and the black hole spin $J=0.998$ is depicted in Fig. 9. The inspiral starts at four gravitational radii.

The EMRI events from the Galactic center will be within the detection sensitivity limits of the planned LISA and eLISA space-base interferometers that will be capable to detect the gravitational wave events with low frequencies in the range $\nu_{\mathrm{GW}}=0.1 \mathrm{mHz}-1 \mathrm{mHz}$. Although the likelihood to detect an EMRI event for the Galactic center is rather low during the mission lifetime, Gair et al (2004) estimate that LISA will be able to detect $\sim 2$ EMRI events of $1.4 M_{\odot}$ compact objects (white dwarfs and neutron stars) per cubic Gpc per year for black holes with similar masses as $\operatorname{Sgr} \mathrm{A}^{*}$.

A very rare but rewarding event would be the detection of an inspiraling pulsar emitting gravitational waves. Such an event would allow us to do two independent measurements: An electromagnetic based timing measurement, comparing the results with the general relativity predictions, and the other, a GW-based measurement - also in comparison with theory. With a mass ratio of $m_{\star} / M_{\bullet} \sim 2 \times 10^{-7}$ this experiment is at the sensitivity limit and would only be possible for the Galactic centre, since for other nuclei, pulsars would be too weak to be detected in the radio. Basically the likelihood of this event is mainly given by the likelihood of detecting a pulsar on a relativistic orbit. In principle such a pulsar would already be emitting GWs with the flux depending on its semi-major axis (see equations in the appendix D). 

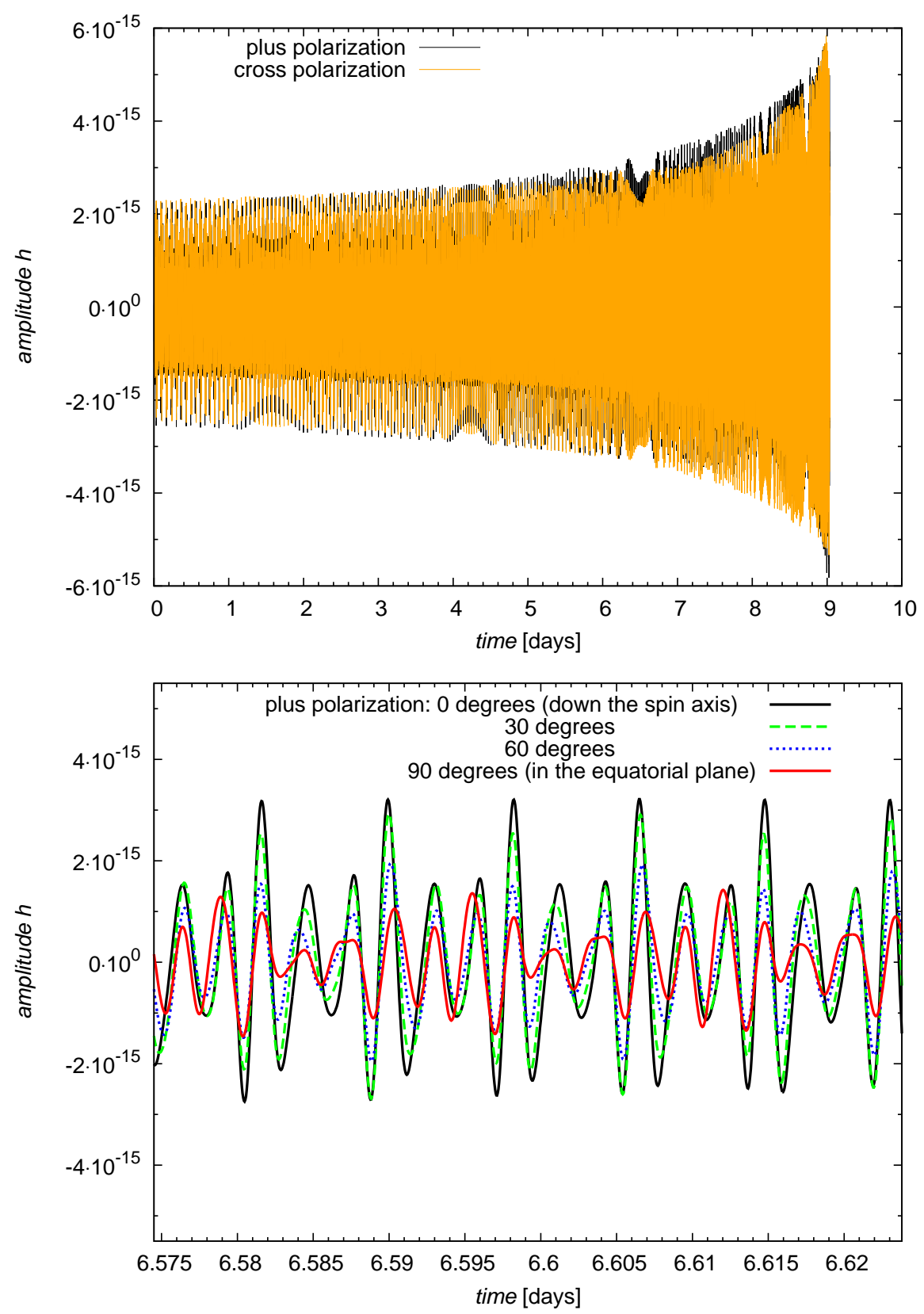

Fig. 9 Top: A waveform for an EMRI event starting at $\mathrm{t}=0$ days at four gravitational radii for $m_{\star} / M_{\bullet}=10^{-4}$ (black hole spin 0.998 ) as viewed at $30^{\circ}$ from the spin axis of the black hole. The event corresponds to a non-equatorial orbit inclined at $40^{\circ}$. Two polarization modes (plus and cross) are depicted by different colors. The data for the plot were taken from the simulations of Hughes (2000, 2001).

Bottom: Zoom into the waveform around $t=6.6$ days. Different lines represent a different viewing angle (see the key). 
4.14 Manipulative success

We may ask if the concept of the "manipulative success" presented in the framework of Entity Realism in section 3 can be applied to SMBHs. Of course, we cannot "use" and "manipulate" them, but this is the case for almost all phenomena studied in astrophysics. The problem, however, is solvable statistically. In this sense, we can study candidates for SMBHs in different environments (i.e. in nuclei of galaxies of different types and masses); hence, we can quasi exploit them in a statistical way by observing samples of SMBHs and $\mathrm{SgrA}^{*}$ at the center of the Milky Way is one of them. Examples for such studies are the scaling relations for SMBHs (Graham et al, 2015; Ferré-Mateu et al, 2015), merger scenarii for gravitational waves detection (Schnittman, 2013), reverberation analyses of black hole masses (Shen et al, 2015; Matsuoka et al, 2015), recoil scenarii in galactic nuclei (Markakis et al, 2015; Guedes et al, 2011).

All of these phenomena need large compact masses as they are provided by SMBHs. It is, however, currently difficult to distinguish between an action induced by a SMBH or a very compact boson star (see section 5.2). To use the principle of "manipulative success" we need observables that directly address the presence of an event horizon. Here, jet launching mechanisms and QPO's (quasi-periodic oscillations) originating close to ISCOs (innermost stable orbits of matter orbiting black holes) appear to be suitable candidates especially for large SMBHs in the sky located in radio loud AGN. There, the advanced properties of mm-VLBI as expected to emerge from the EHT can be useful.

\section{Alternatives to the Black Hole Scenario}

If an alternative scenario described the observed phenomenon better, the consequences for astrophysics would be dramatic. Depending on the model, this could effect the evolution of supernovae, the merging of binary black holes, evaporation of black holes, gravitational wave emission and gravitational wave background. The question whether the Galactic Center black hole is a black hole has far reaching astrophysical consequences because it is the best case for a black hole. As discussed in section 2.3. already for bona fide black holes quantum processes are of relevance. The alternative scenarii that are discussed in the following fully rely on quantum phenomena. The so called "fermion ball" and the "boson star" scenarii, discussed in the following subsections cover important "dark particle matter" models that have been under discussion as an alternative to the central Galactic black hole. A critical discussion on constraints on alternative models to SMBHs is given by Miller (2006). Here, we will briefly summarize their properties and discuss whether they are suitable descriptions of the extreme mass concentration found at the position of SgrA* (see also Fig 6). In sections 5.3 and 5.4 we also mention the possibility of objects such as grava-stars and macro-quantumness as interesting concepts for interpretation and understanding the upcoming observational data. Here, we will not refer to wormholes that have been shown to be unstable by Homer Ellis in 1973 (Ellis, 1973).

\subsection{Fermion Ball}

The fermion ball as an attempt to explain large compact nuclear masses observed at the centers of galaxies was introduced by Viollier et al (1992) and De Paolis et al (2001). A motivation for the development of the neutrino ball scenario was a decreasing radiative efficiency towards the center of the Milky Way. This could be 
linked to a resolved mass - and therefore a decreasing gravitational potential near the very center. Such a scenario would help to explain the low luminosity of Sgr A*. In the specific case of a fermion ball, these objects are stabilized by the degeneracy pressure of e.g. neutrinos as the corresponding fermion candidates. Due to the Pauli principle, the degeneracy pressure of the fermions can be balanced by the self-gravity of a ball of degenerate fermions. In this case, the non-relativistic LaneEmden equation can describe the relation between the mass $M$ and the radius $R$ of a fermion ball, composed of fermions with mass $m$ and degeneracy $g$. The maximal mass for a degenerate fermion ball, can be calculated in a general relativistic framework. The maximum mass of such a degenerate fermion ball is given by the Oppenheimer-Volkoff limit $M_{O V}$. For a given fermion mass $m$, all objects heavier than $M_{O V}$ must then be black holes (Viollier et al, 1992).

It is very difficult to explain all SMBHs like SgrA* at the low end and e.g. M87 at the upper end with the same fermion mass and degeneracy (Viollier et al, 1992). M87 (e.g. Walsh et al, 2013) is one of the most massive central dark objects currently known, with a mass of $\sim 6.3 \times 10^{9} \mathrm{M}_{\odot}$. This implies a fermion mass around $17 \mathrm{keV} / \mathrm{c}^{2}$. For a few objects one even infers masses that are almost a magnitude higher than that of M87 (Walker et al, 2014; Ghisellini et al, 2009, 2010)) implying even smaller fermion masses. A small fermion mass of $17 \mathrm{keV} / \mathrm{c}^{2}$ will result in a fermion ball radius of about 10 light days, or at the distance of $\mathrm{SgrA}^{*}$, about 8.3 mpc. In this case, large sections of the S2 stellar orbit would be located inside the fermion ball. The orbit of S2 would be expected not to be closed and would be subject to a significant Newtonian periabothron shift, since the extended fermion ball mass would be resolved by the orbit. This is due to the fact that the effective gravitational attraction the star is exposed to, changes towards smaller distances to the center. Hence, it appears that one can exclude the possibility that all compact dark objects at the centers of galaxies can be explained by a universal neutrino ball model.

On the other hand, the stellar orbit of S2 (Gillessen et al, 2009a; Horrobin et al, 2004; Eisenhauer et al, 2003) and the fact that no significant Newtonian peribothron shift has been found constraints the fermion mass and implies that it should be higher than $400 \mathrm{keV} / \mathrm{c}^{2}$ (e.g. Viollier et al (1992); Bilic et al (2003)). Hence, it is very difficult to explain all SMBHs like SgrA* at the low end and e.g. M87 at the high end with the same fermion mass and degeneracy (Viollier et al, 1992). This makes the concept of a neutrino ball not to appear very attractive.

In addition, in the case of $\operatorname{SgrA}^{*}$ a neutrino ball could not account for the compactness of the SMBH observed at radio/mm wavelengths. Observations of X-ray and NIR flares from Sgr A* (Mossoux et al, 2015; Neilsen et al, 2015b; Barrière et al, 2014; Eckart et al, 2012c; Porquet et al, 2008; Eckart et al, 2006a; Ghez et al, 2004; Eckart et al, 2003; Genzel et al, 2003; Baganoff et al, 2003, 2001) suggest that the emission comes from structures smaller than about ten Schwarzschild radii of a $\sim 4 \times 10^{6} \mathrm{M}_{\odot}$ million solar mass black hole. This is more than two orders of magnitude more compact than the radius of a neutrino ball with a neutrino mass discussed in this context.

A further significant drawback of that scenario is that it does not explain what happens to the permanently in-falling baryonic matter. This matter will of course be trapped and concentrate at the bottom of the potential well. This will unavoidably result into a seed black hole at some point. This scenario then defeats the purpose of having a ball of degenerate matter (especially neutrinos; Melia and Falcke (2001)). 


\subsection{Boson Ball}

The boson star scenario is another model that could explain a very large and compact mass, yet it is much more speculative than black holes. It is a dark matter particle explanation that cannot easily be ruled out by the present data. Such a ball of Bosons could be present in a very compact configuration that is difficult to distinguish from a black hole in terms of compactness (i.e. size). However, it is hard to understand how the bosons managed to cool sufficiently in order to settle down into such a small volume and do not form a black hole during that process $\mathrm{MaOz}$ (1998). Boson stars (Kaup, 1968) are supported by the Heisenberg uncertainty principle. Ruffini and Bonazzola (1969) showed that - e.g. for a boson mass of $1 \mathrm{GeV} / \mathrm{c}^{2}$ - a stable object of total mass of $10^{-19} \mathrm{M}_{\odot}$ and $1 \mathrm{fm}$ diameter could be formed. Obviously, the mass depends on the repulsive forces between the bosons. If one wants to form objects with total masses as large as they are found in galactic nuclei (Colpi et al, 1986), then one must ad hoc introduce a hypothetical weak repulsive force between bosons (Colpi et al, 1986). For a large range of hypothetical boson masses, they can have sizes of only several times their Schwarzschild radii. This is the prime reason why it is so difficult to clearly distinguish observationally between compact boson stars and black holes as candidates for supermassive objects as they are found at the nuclei of galaxies (see also Torres et al, 2000; Mielke and Schunck, $2002,2000)$.

However, during its lifetime, even if a boson star had formed at the center, it should eventually have collapsed to a black hole through accretion of the abundant gas and dust in the Galactic Center. Therefore we conclude that similar to the fermion ball solution, a supermassive boson star is not an astrophysically attractive explanation for the high mass concentration at the center of the Milky Way. As for possibilities of definitely ruling out the boson star scenario, simultaneous multiwavelength measurements of the emission from Sgr A* (e.g. Eckart et al, 2012d) will allow us to constrain the emission mechanism and therefore the compactness of the emitting region around Sgr A* even further. Probably within the next decade it will be possible to image the "shadow" cast by the putative black hole through deflection of light rays using global radio interferometry at sub-millimeter wavelengths. Such an experiment will involve very long baseline interferometry in the sub-mm regime (Melia and Falcke, 2001; Falcke and Markoff, 2000). However, Vincent et al (2015) show that relativistic rotating boson stars can result in images that look very similar to those expected from Kerr black holes, also revealing shadow-like and photonring-like structures. This demonstrates that it is very challenging to unambiguously discriminate the presence of a black hole with an event horizon from other highly concentrated mass agglomerations.

The future for Galactic Center research lies in high angular resolution observations at all accessible wavelengths. For radio wavelengths, progress will be made with VLBI at mm-wavelengths. In the infrared wavelength domain interferometry is now possible with large aperture interferometers that will - in the near future allow us to observe $\mathrm{SgrA}^{*}$ with a resolution of a few milliarcseconds. These are the Very Large Telescope Interferometer, the W.M.Keck Interferometer, and the Large Binocular Telescope. (Pott et al, 2005; Eckart et al, 2006c; Pott et al, 2008) have carried out first mid-infrared interferometric observations of a number of bright $10 \mu \mathrm{m}$ sources within the central stellar cluster using MIDI Leinert and Graser, 1998) and the $47 \mathrm{~m} \mathrm{UT} 2 / \mathrm{UT} 3$ baseline. In these observations, fringes on the first Galactic Center source were obtained on the stellar source IRS3. In the very near future further infrared interferometer measurements in the Galactic Center area will be possible (Eisenhauer et al, 2008; Eckart et al, 2010, 2012d; Vincent et al, 2011a).

The alternative explanation of the central mass as a massive boson star (Torres et al (2000); Mielke and Schunck (2000); Lu and Torres (2003) and references therein) is 
severely challenged by the good agreement between the measured polarized flare structure and the theoretical predictions (Gillessen et al, 2006; Eisenhauer et al, 2005; Broderick and Loeb, 2005; Ghez et al, 2004) as well as the indication of a quasi-periodicity in the data. If an ad hoc weak repulsive force between a hypothetical brand of bosons is introduced, it appears to be possible to form massive, compact objects with sizes of a few $\mathrm{R}_{S}$ that are supposedly supported by the Heisenberg uncertainty principle. However, it is a delicate process to form a boson star and preventing it from collapsing to a MBH despite of further accretion of matter, a non spherically symmetric arrangement of forces as in the case of a jet or matter being in orbit around the center but well within the boson star. Such a massive boson star scenario could already be excluded for the nucleus of MCG-6-30-15 (Lu and Torres, 2003 ). Here, of the $\mathrm{K}_{\alpha}$ line emission of the relativistically moving plasma of the accretion disk sourcounding the object could be used to put constraints on the mass concentration.

The results from Vincent et al (2015) show that for relativistic rotating boson stars it may still be very difficult to discriminate a boson star from a SMBH. In the case of a stationary boson star, the orbital velocity close to the $\sim 3 \mathrm{R}_{S}$ radius of the last stable orbit is already $\sim 3$ times lower than that of a Schwarzschild MBH (Lu and Torres, 2003) and relativistic effects are severely diminished and further reduced at even smaller radii. If the indicated quasi-periodicity is due to orbital motion then a stationary boson star can be excluded as an alternative solution for $\mathrm{SgrA}^{*}$, since in this case one expects the orbital periods to be larger. However, if pulsars on highly eccentric orbits close to SgrA* can be found, then the black hole versus boson star riddle may be solvable by measuring the central massive object's quadrupole moment (e.g. Psaltis, 2012). How Kerr black holes and boson stars may be different with respect to their quadrupole moments is described in detail in (e.g. Herdeiro and Radu, 2014). Whether such a measurement is successful will also depend on the environment and orbital disturbances.

\subsection{Grava-stars}

The grava-star model postulates a strongly correlated thin shell of anisotropic matter surrounding a region of anti-de Sitter space. It has been proposed as an alternative to black holes first by Mazur and Mottola (2001). Broderick and Naravan (2007) discuss the grava-star model for SgrA* but conclude that present day astronomical observations rule out modifications of GR of the kind described by Chapline (2003) on all scales larger than the Planck length.

One observational consequence of the analysis presented by Broderick and Naravan (2007) is that (in particular) in the case of $\mathrm{SgrA}^{*}$, a grava-star will not have had sufficient opportunity to cool if the accretion is continuous. However, if mass accretion is predominantly done through stellar capture events during a rather transient rapid accretion, this would allow us $\mathrm{SgrA}^{*}$ to cool over timescales between captures of the order of $10^{4}$ years. While occasional stellar captures may occur, the current observational evidence suggests a rather continuous mass accretion at least over the past $10^{5}-10^{6}$ years given the large number massive Helium stars that lose $\sim 10^{-3} \mathrm{M}_{\odot} \mathrm{yr}^{-1}$ into the deep gravitational potential well of SgrA*. The radiatively inefficient accretion flow associated with SgrA* (see section 4.6) suggests that only between $10^{-8}$ and a few times $10^{-10} \mathrm{M}_{\odot} \mathrm{yr}^{-1}$ reach the SMBH (Broderick and Loeb, 2006; Yuan et al, 2003; Narayan et al, 1995). However, a clearer distinction between the black hole and the grava-star case is probably within reach using high-resolution VLBI observations in the future (Sakai et al, 2014). 


\subsection{Macro-quantumness}

There could also be yet unknown observational effects of a strength depending on whether the composition of the black hole is rather baryonic or not. Dvali and Gomez (2011) propose that Galactic black holes could be quantum objects i.e., BoseEinstein condensates of $N$ soft gravitons at the quantum critical point, where $N$ Bogoliubov modes become gap-less (see also Dvali and Gomez, 2013; Dvali et al, 2013). Thus, predictions from semi-classical physics, usually applied to describe black holes, might not provide the proper descriptions. The metric itself might become an approximate entity. In the following we assume that the black holes carry a quantum memory about their baryonic content. This is mainly based on the quantum picture and model independent arguments. In case of baryonic content, this is usually referred to as "baryonic hair". It is still under discussion what the observable macroscopic effects are (e.g. Cunha et al, 2015), but one can already argue about the relative strength of these effects (see also Dvali et al, 2016): It seems that observable macroscopic effects of the "baryonic hair" are suppressed by the ratio $N_{B} / N$, where $N_{B}$ is the baryon number of a black hole, and $N$ is the occupation number of gravitons, which is equal to black hole entropy, i.e., $N=M^{2} / M_{P}^{2}$, where $M$ is black hole mass and $M_{P}$ is the Planck mass. One may assume that this ratio must be small for large black holes even if most of their initial mass is baryonic. In this case, $N_{B}=M / m_{b}$ ( $m_{b}$ being baryon mass) will be a very small number as compared to $N$, if the black hole radius is much larger than $1 / m_{b}$. On the other hand, the effects of "baryon hair" may be strong as baryons interact stronger than gravitons. This leaves us with the conclusion that the observable effects from baryonic hair are expected to be very strong for very small black holes (of mass $10^{14} \mathrm{~g}$ or lighter). Therefore, it cannot be excluded with certainty that the conventional classical no-hair description is valid to a good accuracy for black holes that are much heavier than $10^{14} \mathrm{~g}$, provided they are formed as a result of collapse of predominantly baryonic matter. In this case, the effects from baryon hair will most likely be very small for massive black holes. However, if one allows that the heavy black hole content could be non-baryonic and that a black hole can form from some exotic light particles (e.g. dark matter of light sort), the situation may be very different. In case these light particles have a mass comparable to the inverse gravitational radius, one can show that the observational effects are very strong for large black hole masses. It is unclear what these super-light hypothetical particles could be. It should, however, be pointed out that this assumption is not any more exotic than the assumption of boson stars for which the hypothetical existence is based on the postulated existence of new particle species and interactions amongst them.

\section{Future progress in observations}

The combination of existing and planned millimeter/submillimeter facilities into the Event Horizon Telescope (EHT) 18 will give a high-sensitivity, high angular resolution of better than $60 \mu$ as. In the more distant future the EHT array may be supported by a mm-satellite facility in space to increase the angular resolution by factors of a few (see Millimetron Space Observatory; a $10 \mathrm{~m}$ aperture cooled telescope Kardashev et al (2007, 2014)). In particular the phased Atacama Large Millimeter Array (ALMA) 19 , in Chile will substantially contribute to this development. Over the current decade, this instrument will finally allow us to directly probe the event horizon of the black hole candidates SgrA* and M87. This requires the

18 http://www.eventhorizontelescope.org/

19 https://www.eso.org/sci/facilities/alma.html 
deployment of submillimeter dual-polarization receivers and highly stable frequency standards. Such an equipment will finally enable us to carryout VLBI experiments at $230-450 \mathrm{GHz}$ in oder to perform a detailed and direct search for the edge of black hole. At longer radio wavelengths, in the future the Square Kilometre Array (SKA 20 ) will be sensitive enough to detect, pulsars in a close orbit $\left(P_{\text {orb }}<1 \mathrm{yr}\right)$ around $\mathrm{Sgr}$ $A^{*}$ and most importantly measure its timing properties, which would enable to test "cosmic censorship conjecture" as well as the "no-hair" theorem (Psaltis et al, 2016b, a; Eatough et al, 2015).

At near-infrared wavelengths, the GRAVITY21 experiment at the Very Large Telescope Interferometer (VLTI) of the European Southern Observatory (ESO) will allow us to carry out precision narrow-angle astrometry in the ten microarcseconds and interferometric imaging in the milliarcsecond regime. GRAVITY will allow us to use dual beam phase reference observations of SgrA* and will therefore as a second generation VLTI instrument enhance the sensitivity and accuracy far beyond todays limits. GRAVITY will be able to measure the motion of the photo-center of the SgrA* image during flares. If the flares are linked to orbital motion of a hot-spot within a temporarily bright accretion disk, GRAVITY will see the periodic oscillations of the centroid position. Similarly, bright outflow components that separate from $\mathrm{SgrA}^{*}$ can be detected and distinguished from components within the accretion disk.

Across the wavelength domain new instrumentation like the James Webb Space Telescope (JWST) 22 or the Constellation X 23 and Xeus X-ray 24 satellite missions will provide sensitive information on the spectral shape and variability of SgrA*. For instance $\mathrm{SgrA}^{*}$ has not yet been detected in the mid-infrared wavelength domain longward of about $5 \mu \mathrm{m}$ wavelength. The high point source sensitivity and foreseeably stable point spread function of the JWST will allow for progress. The combination of high sensitivity and imaging quality will also help to investigate the nature of faint X-ray flares that are currently difficult to measure due to low count rates and contamination of a prominent Bremsstrahlung source surrounding SgrA*. The investigation of faint flares and the comparison with flares at different wavelengths may shed light on the origin of the X-ray flare emission (pure Synchrotron or SSC?) and hence on the energetics in the immediate vicinity of the black hole candidate $\operatorname{Sgr} A^{*}$.

Recent progress in detecting gravitational waves from merging black hole binaries (Abbott et al, 2016) using LIGO25 shows that this window is also opening for exploring the immediate vicinity of $\operatorname{Sgr} A^{*}$. The interaction of stars and stellar remnants amongst each other as well as with the large mass at the center of the Milky Way may provide tools to explore the nature of SgrA* using gravitational waves (e.g. Amaro-Seoane et al, 2012; Freitag, 2003; Pierro et al, 2001).

\section{Synthesis: Combining the Results}

We can now proceed to apply the Eleatic Priciple to the investigation of SgrA* and then ask the question: How good a case for being a SMBH is it and how good a case can it become? Based on the preceding discussion we have described a number of critical observational results that are required as necessary conditions of calling

\footnotetext{
20 The Square Kilometre Array (SKA) will be the worlds largest radio telescope, with a collecting area of more than one square kilometre (one million square metres); https://www.skatelescope.org/

21 https://www.eso.org/sci/facilities/develop/instruments/gravity.html

22 http://www.jwst.nasa.gov/

23 http://constellation.gsfc.nasa.gov/

24 http://www.rssd.esa.int/index.php?project=XEUS

25 http://www.ligo.org/science.php
} 


\begin{tabular}{|c|c|c|}
\hline label & necessary condition & $\begin{array}{l}\text { referring to } \\
\text { section }\end{array}$ \\
\hline$N_{1}$ & Is object at nominal position of SgrA*? & 4.1 \\
\hline$N_{2}$ & Is size of emitting region in $\mathrm{SgrA}^{*}$ sufficiently small? & 4.3 \\
\hline$N_{3}$ & Is mass of $\mathrm{SgrA}^{*}$ in agreement with SMBH masses? & 4.2 \\
\hline$N_{4}$ & Does the distance to SgrA* place it at the center of the Milky Way? & 4.1 \\
\hline$N_{5}$ & Is the manipulative success for $\mathrm{SgrA}^{*}$ similar to other $\mathrm{SMBH}$ candidates? & 4.14 \\
\hline$N_{6}$ & Is a bright fast jet originating from $\operatorname{SgrA}^{*} ?$ & 4.8 \\
\hline$N_{7}$ & Do we detect a merger ringing signal in gravitational waves from SgrA*? & 4.13 \\
\hline$N_{8}$ & Do we detect an exceptionally bright flare from SgrA*? & 4.7 \\
\hline$N_{9}$ & Do stars and pulsars close to SgrA* give indications for a SMBH? & 4.11 \\
\hline$N_{10}$ & Is the spectrum of the surroundings of SgrA* what es expect from a SMBH? & 4.6 \\
\hline$N_{11}$ & Do we detect a photon ring in SgrA* in addition to orbiting matter? & 4.9 \\
\hline$N_{12}$ & Do VLBI images of SgrA* show a shadow as expected for a SMBH? & $4.10,4.9,4.8,6$ \\
\hline$N_{13}$ & Do we detect photo-center motion of SgrA* with NIR- and/or mm-radio-interferometry? & \\
\hline$N_{14}$ & Can we differentiate fo SgrA* between jet components and hot-spot? & $4.4,4.96$ \\
\hline
\end{tabular}

Table 3 Table of possible necessary conditions that can be combined to result in a sufficient condition required to call SgrA* a SMBH. The necessary conditions have been formulated as logical entities for which we can attribute the locigal values "true" or "false" within the theoretical predictions for supermassive black holes in section 2

SgrA* a SMBH. A priori it is not clear if all necessary conditions are known such that their simultaneous fulfillment results in a sufficient condition for the existence of a SMBH. The conditions discussed in this article are listed in Tab 3 , Conditions $\mathrm{N}_{1}$ to $N_{4}$ are rather fundamental, one might even say, "technical" conditions that are required for most of the combinations to form sufficient conditions. Here, basically only the necessary conditions $N_{5}$ to $N_{8}$ have the character of a "smoking gun", in the sense that a certain observational criterium of spectacular nature points at the existence (or rather "action") of a supermassive black hole. Conditions $N_{9}$ to $N_{14}$ are observational results that are substantially more difficult to obtain. They rely on either special instrumentation and/or the source SgrA* being "cooperative", in the sense that the required event occurs very rarely and/or the black hole environment must be very clean to observe the necessary expected or predicted phenomena. Certainly, not all of these need to be or - given their probability of occurrence - can be combined and fulfilled to serve as a convincing sufficient condition for calling $\mathrm{SgrA}^{*}$ a SMBH. To a certain extent this relieves us from the pressing question if all necessary conditions for the proof of existence are known and fulfilled - as a variety of choices can be used to result in a sufficient condition for the existence for an SMBH at the center of the Milky Way.

In combining the different necessary conditions listed in Tab3 we can first ask the question if there are any conditions that can be extracted from specific combinations of sufficient conditions following equation (9). Trivially, condition $N_{1}$ must always be fulfilled, as the aim is making a statement on SgrA*. However, as we will see, this appears to be the only general condition, but the requirements on the accuracy with which the light at different wavelengths can be associated with the position of the large mass varies strongly from case to case. We also note that not all conditions listed in Tab 3 are strong necessary conditions, e.g. the spectrum $\left(N_{10}\right)$ of the surroundings of SgrA* may equally well be produced by other scenarii involving a very compact massive object that is not necessarily an SMBH. A schematic representation of the different combinations discussed in the following is shown on the left side of Fig. 3 .

Possible combinations that may lead to convincing sufficient conditions appear to be:

$S_{1}=N_{1} \wedge N_{2} \wedge N_{3} \wedge N_{4} \wedge N_{5} \wedge N_{10}$ : The "manipulative success" can only work in a statistical sense and is difficult to be applied to $\operatorname{SgrA}^{*}$ as an individual source. However, if solid observables for demonstrating "manipulative success" can 
be found and $\mathrm{SgrA}^{*}$ is included in the sample, then this could be looked upon as a sufficient condition. Depending on these observables mass, the position and distance will be needed. In addition to mass and distance, the overall spectrum and a small radio source size will be needed to support the presence of a SMBH in the framework of a statistical compilation centered around the "manipulative success" approach.

$S_{2}=N_{1} \wedge N_{3} \wedge N_{4} \wedge N_{6}$ : The detection of a jet showing relativistic properties like superluminal motion of individual components is a clear indication of an accretion phenomenon onto a very compact massive object. It needs to be combined with the presence of a large mass to be indicative of a SMBH identification. Obtaining the mass needs the position of SgrA* and its distance. A strong and fast jet implies strong accretion which makes it unlikely for fragile objects like neutrino and boson stars to persist and not to collapse into a SMBH.

$S_{3}=N_{1} \wedge N_{8}$ : The detection of an exceptionally bright flare from SgrA* that may compensate a major part of its current underluminosity of $10^{-9}$ times the Eddington luminosity and would certainly indicate the presence of a strong accretion event onto a very compact object like a SMBH. Such very bright outbursts of SgrA* have apparently occurred in the past. A bright X-ray outburst of SgrA* within the recent $\sim 400$ years is being discussed as the possible reason for the X-ray fluorescence emission from massive molecular clouds that are surrounding the Galactic Center (Clavel et al, 2013; Terrier et al, 2010; Revnivtsev et al, 2004; Sunvaev and Churazov, 1998). Witzel et al (2012b) explain this $\left(\sim 10^{39} \mathrm{erg} / \mathrm{s}\right)$ outburst via an extreme value of their near-infrared statistics without the need for an extraordinary event. Based on the flux frequency plot for SgrA* shown in Fig.19 of Witzel et al (2012b) and the conditions discussed therein, the maximum near infrared brightness expected at $2 \mu \mathrm{m}$ wavelength would be of the order of $3 \mathrm{Jy}$ or about 8.5 magnitudes. It would occur every 10 to 100 million years.

$S_{4}=N_{1} \wedge N_{2} \wedge N_{4} \wedge N_{11}$ : If the accretion process is clean enough, one can speculate that in addition to orbiting matter a secondary photon ring can be detected towards Sgr A*. Imaging of a photon ring could be done as a quasi-stationary image taken with VLBI at short mm-wavelengths resulting in assessing the small intrinsic source size with minimum influence by scattering effects. Modeling results using the distance to SgrA* will result in a mass and compactness and will allow us to state that a sufficient condition for the detection of an extremely high mass concentration like that provided by a SMBH has been fulfilled. However, Vincent et al (2015) show that it may not be possible to uniquely conclude that it is in fact a SMBH rather than a boson star. Differences between the images of the secondary photon ring expected for a black hole and a boson star require VLBI imaging with a high dynamic range and the source being in a low state of activity to allow for sensitive imaging.

$S_{5}=N_{1} \wedge N_{7}$ : The detection of a merger ringing signal in gravitational waves is a clear indication for a highly relativistic phenomenon. The ringing frequency would allow us to pinpoint the evolved masses and size scales without previous knowledge of these quantities. Ringing indicates a merger process and hence violent accretion which makes it unlikely for fragile objects like neutron or boson stars to remain stable (see above). However, the small expected merger rates make the detection of a ringing signal very unlikely. In this context, it is the comparison to the observational results for other galactic nuclei which may help to understand the situation for SgrA*.

$S_{6}=N_{1} \wedge N_{9}$ : If the search for stars and pulsars towards SgrA* is successful, then the mapping of spacetime will tell the mass and size scales. The orbital parameters of stars and pulsars will allow us to independently determine the distance and involved size scales. Detailed mapping of spacetime with pulsars or stars within a few 1000 Schwarzschild radii and measuring the quadrupole moment of SgrA* (e.g., Psaltis, 2012; Psaltis et al, 2016a) also allows us to convincingly distin- 


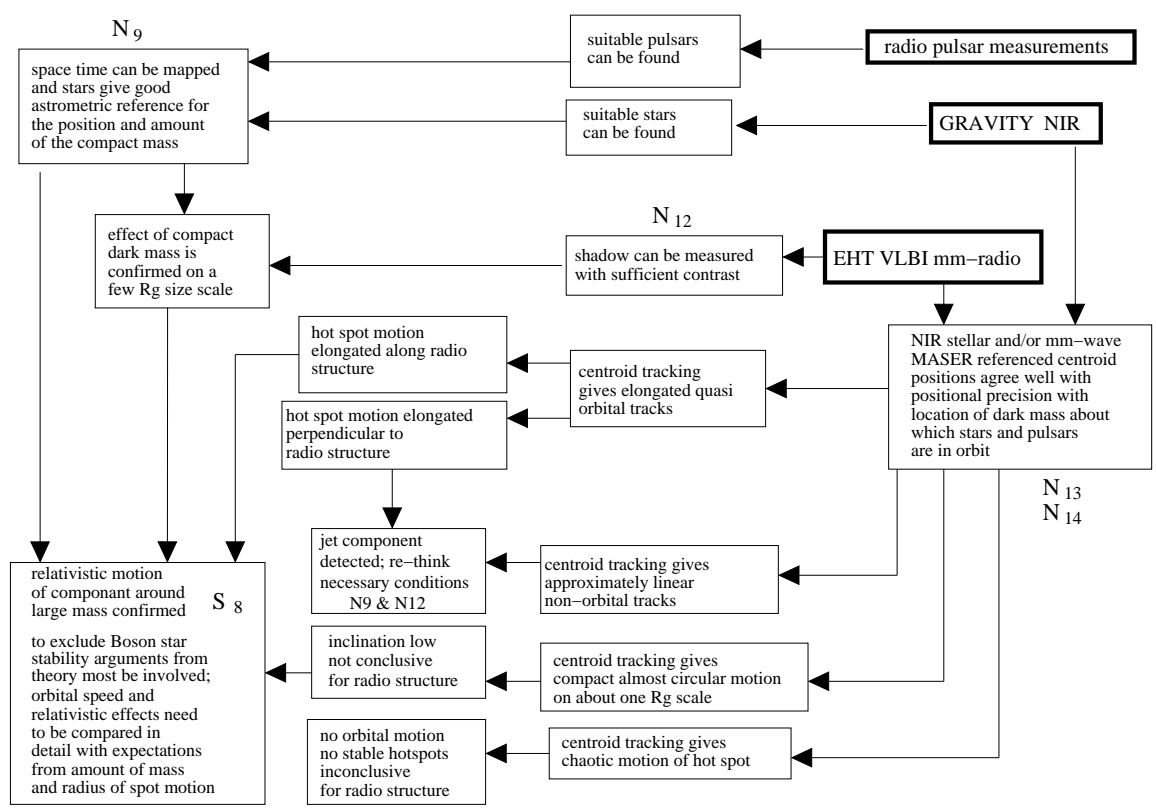

Fig. 10 Sketch of a decision flow chart for option $S_{8}$ to combine suitable necessary conditions.

guish the black hole case from a boson star case. If GRAVITY finds stars with small separations to SgrA*, this may be the only experiment that allows us to simultaneously indentify the location of the SgrA* continuum emission with the position of the central mass, i.e. the object about which the stars are in orbit. This seems to be within reach even with the the currently observable stars S2, S02-1, and S38 (Boehle et al, 2016; Parsa et al, 2017).

$S_{7}=N_{1} \wedge N_{2} \wedge N_{12} \wedge N_{13} \wedge N_{14}$ : Combining mm-VLBI and: NIR-interferometry results: mmVLBI images will be very compact and croissant shaped both for orbiting spots and for jet components (Dexter et al, 2012b, a ), because the formation of a sensitive VLBI image takes at least several hours and this is largely in excess of the orbiting time scale of spots close to the ISCO (less than about half an hour) in the case of SgrA*. Hence, VLBI will deliver at best a quasi-stationary image of the shadow which could be resulting from a jet dominated image as well (Dexter et al, 2012a, b). It is not clear, how well low contrast spots orbiting the central mass can be tracked with a high time resolution using VLBI. A limited and variable uv-plain coverage as well as radiative transport phenomena may make it difficult to reliably produce densely sampled time series of snapshot images. Also, Vincent et al (2015) show that shadow-like structures can be produced by other extremely high mass concentrations like e.g. boson stars. SgrA* is variable at all wavelengths indicating variable accretion and possibly structural changes in the continuum light emitting central region. Hence, it is unclear under what conditions small differences between the expected shadow and accretion disk structure between a black hole and a boson star scenario can be detected with VLBI imaging.

The NIR GRAVITY experiment will not be able to spatially resolve the immediate vicinity (1-2 mas; see below) region around $\operatorname{SgrA}^{*}$ (i.e. its accretion wind or a temporary disk/jet structure). The resolution may not be sufficient to determine if the emission is coming from the ISCO or from a jet nozzle that may be significantly offset from the black hole and hence may not reflect the situation in its immediate vicinity (see the cases of M87 and SgrA* discussed by Nakamura and Asada, 


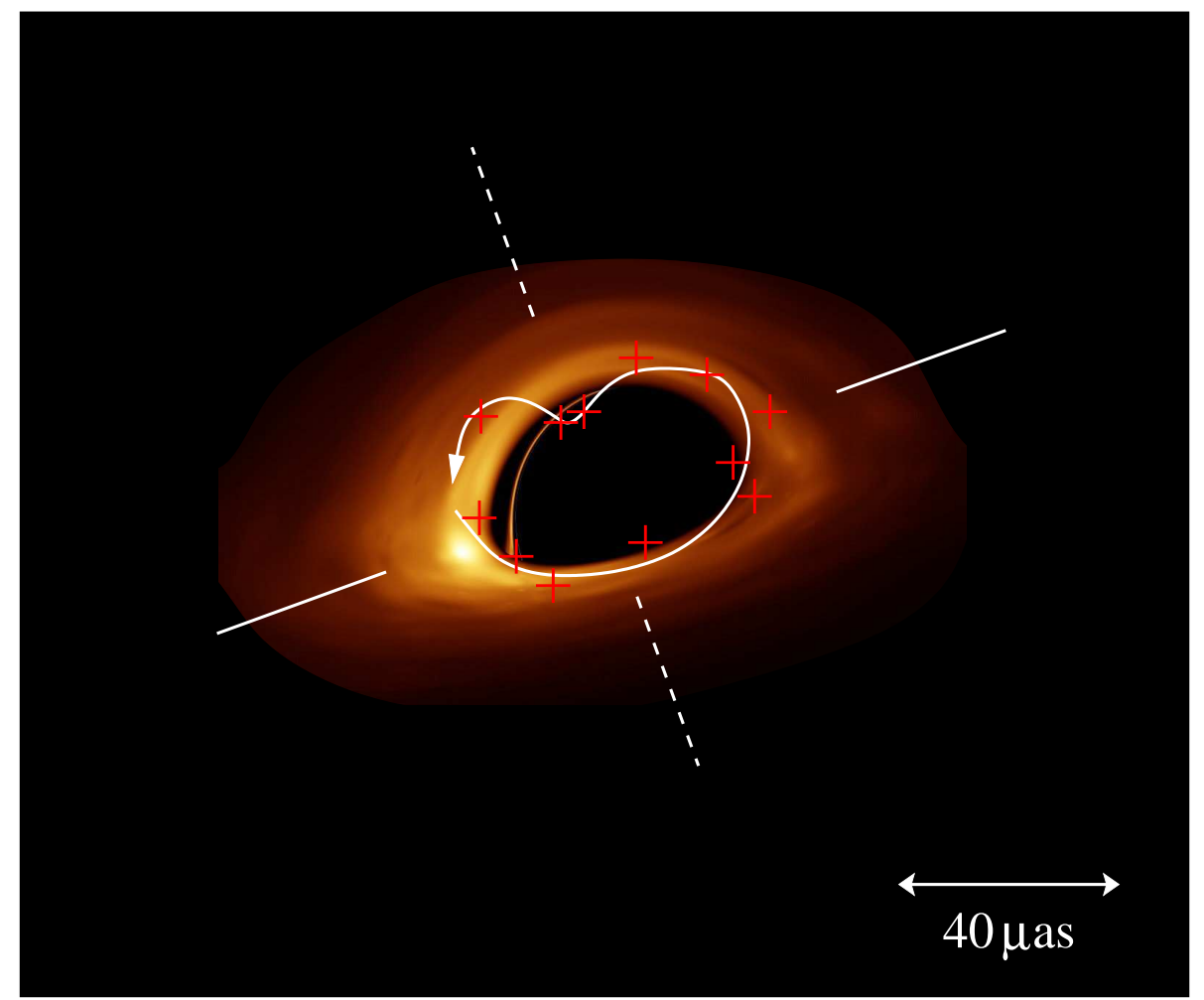

Fig. 11 Photocenter motion compared to a disk model. The example of a NIR photo-center motion as planned to be measured with the GRAVITY interferometer at the VLTI is taken from Paumard et al (2008) and Paumard et al (2005). The simulation describes the apparent trajectory of flare events assuming material orbiting a non-rotation black hole at an inclination of $45^{\circ}$ on the last stable orbit at a distance of $3 R_{S}$ from the center. Lensing (including multiple images), relativistic beaming and Doppler effect are included in the relative positioning of the resulting data points (red crosses) following the orbital track (white line; further details in Paumard et al, 2008). The image (Bursa et al, 2007) is assumed to represent a mm-VLBI data disk model that shows luminous material for radii beyond the last stable orbit. The dashed and straight white arrows indicate the directions perpendicular and along the radio structure that we refer to in the text.

2013; Falcke et al, 2009). In the radio regime such an apparent offset depends on the position at which the jet becomes optically thin. At shorter wavelengths, however, investigations of stellar and SMBHs in the framework of "lamp post" models indicate that the bright base of the jet may be located about 2 Schwarzschild radii (or more) above the accretion disk, i.e. at some distance from the disk's ISCO orbit (Miller et al, 2015; Emmanoulopoulos, 2015). The radio and NIR position of SgrA* agree to within 10 mas (Reid et al, 2003b), i.e. about 1000 Schwarzschild radii. The inferred position agrees with the position of the mass concentration about which the stars orbit with projected separations from SgrA* of about 1 mas i.e. about 100 Schwarzschild radii (Reid et al, 2003b; Ghez et al, 2008; Gillessen et al, 2009a). Hence, there is room for a significant offset between a possible jet nozzle from the ISCO.

However, a great advantage of the NIR interferometric measurements is that it will allow us to track and time resolve the centroid motion of SgrA* with integration times of a few minutes. Following the motion of a hot plasma blob in orbit around (or in transversal motion towards or away from) SgrA* requires being able to distinguish between bright spots separated only a few Schwarzschild radii from each other. This implies an accuracy of a few ten of microarcseconds. Hence, VLBI images alone 

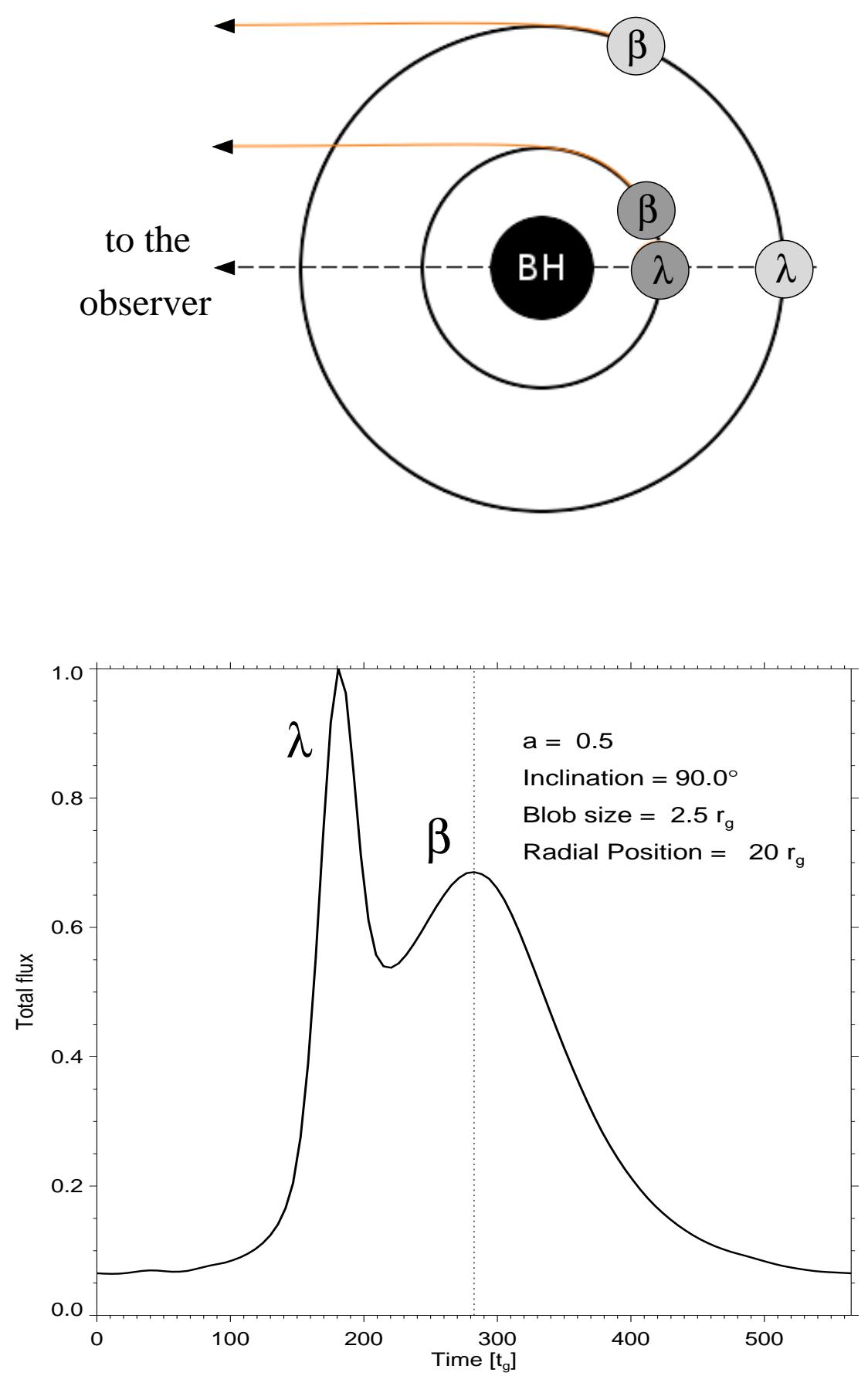

Fig. 12 At the top we show the geometrical arrangement of position at which beaming $(\beta)$ and lensing $(\lambda)$ may lead to detectable subflare structures. These are marked for an example case in the light curve on the bottom. The light curve is plotted for a full orbit of the plot as a function of gravitational time units in $t_{g}=r_{g} / c$. For a black hole mass of $4.3 \pm 0.3 \times 10^{6} \mathrm{M}_{\odot}$ the unit $t_{g}$ is of the order of 20 seconds. 
$\left(N_{1} \wedge N_{12}\right)$ as well as NIR centroid measurements by themselves $\left(N_{1} \wedge N_{13}\right)$ may not allow for differentiation between a foot-point of a precessing jet and an orbiting hot-spot $\left(N_{14}\right)$. However, the NIR result will allow us to discover an oscillating bright spot motion orientated along the VLBI structure (see Fig. 11). This is expected for inclined orbital motion of spots at a distance of a few Schwarzschild radii from the black hole (compare Fig. (7) left and Fig. 8 right as well as Zamaninasab et al (2010) and Vincent et al (2014)). One may expect periodic motion of spots at larger radii perpendicular to the VLBI structure in the case of a foot-point of a precessing jet (see Fig. 11 and 7). The quality of the alignment of the two disk representations in Fig. 11]will depend on the quality of the infrared and radio reference frame. However, perfect alignment (i.e. superposition of the black hole mass locations) is not required in order to distinguish between the different directions of motions. For inclinations larger than about $45^{\circ}$ and orbits close to the last stable orbit the radii of the photocenter motion become more circular and are less well suited for comparison with the radio structure. For inclination of less than $45^{\circ}$ the consecutive flare peaks from boosting and lensing may be observable. In Fig. 12 we show the geometrical arrangement for positions at which these light amplifications occur. In addition, we show the lightcurve for an example case for which the time difference between the two sub-flare events will be of the order of 30 minutes. For the example in Fig. 12 the corresponding positional "jump" between these two events amounts to about $4 \mathrm{R}_{g}$, i.e. about $400 \mu$ as, hence well detectable with the 10-100 $\mu$ as astrometric precision expected for GRAVITY. For smaller orbits the amplification points move closer together. The presence of a clearly defined blob, i.e. hot-spot, as well as moderately low inclinations are required in order to observe this effect. The advantage is that the live time of the blob only needs to be of the order of a fraction of an orbital period. In the decision flow shown in Fig.10 this case would correspond to centroid tracking of (short) elongated quasi orbital tracks which would then be perpendicular to the spin axis. Therefore, a combination of both, mm-VLBI and NIR-interferometry with GRAVITY, will lead to a strong sufficient condition to demonstrate the existence of a heavy mass on the scale of one Schwarzschild diameter, i.e. a black hole.

Stellar MASER emission observed with $m m$-VLBI will also allow us to conduct centroiding experiments. Since the mm-emission is optically thick, those observations will be truly complementary to the centroid experiments on the optically thin NIR emission. $43 \mathrm{GHz}$ and $86 \mathrm{GHz}$ masers have been found in the stellar atmospheres of a number of late type stars in the central stellar cluster in the immediate vicinity of $\mathrm{SgrA}^{*}$ (Reid et al (2003b,a) and Borkar et al. 2016 in prep.). Doeleman et al (1998) find that $86 \mathrm{GHz}$ masers mirror the $43 \mathrm{GHz}$ positions. $\mathrm{SiO}$ masers originate for Mira type sources 2-4 stellar radii i.e. 4-8 AU from the star. The maser spot shell will then be distributed over less than 1 mas at the distance of SgrA*. The 200-800 km baselines allow for an angular resolution of up to $\sim 1 \mathrm{mas}$ at $43 \mathrm{GHz}$ and $86 \mathrm{GHz}$ and allow us to phase reference (or in beam reference) SgrA* with respect to the masers. Hence, phase referenced observations can be used to search for and track orbiting spots as well as low-surface brightness features like jets or out- and inflows.

$S_{8}=N_{1} \wedge N_{2} \wedge N_{9} \wedge N_{12} \wedge N_{13} \wedge N_{14}$ : Psaltis et al (2016a) show that the stellar and pulsar results combined with those of the shadow of the black hole result in a high accuracy test for SgrA* being a SMBH. Accepting that the VLBI images do not easily allow us to track a motion on the orbiting time scale at the ISCO, the combination of the pulsar, black hole shadow and the NIR- and mminterferometry result appear as the most unique combination to convincingly show that a SMBH exists. Measuring the detailed influences of the Kerr metric on the orbits of pulsars close to SgrA*, a boson star as an alternative explanation could also be excluded (Psaltis et al, 2016b, a). In Fig. 10 we show a sketch of a decision 


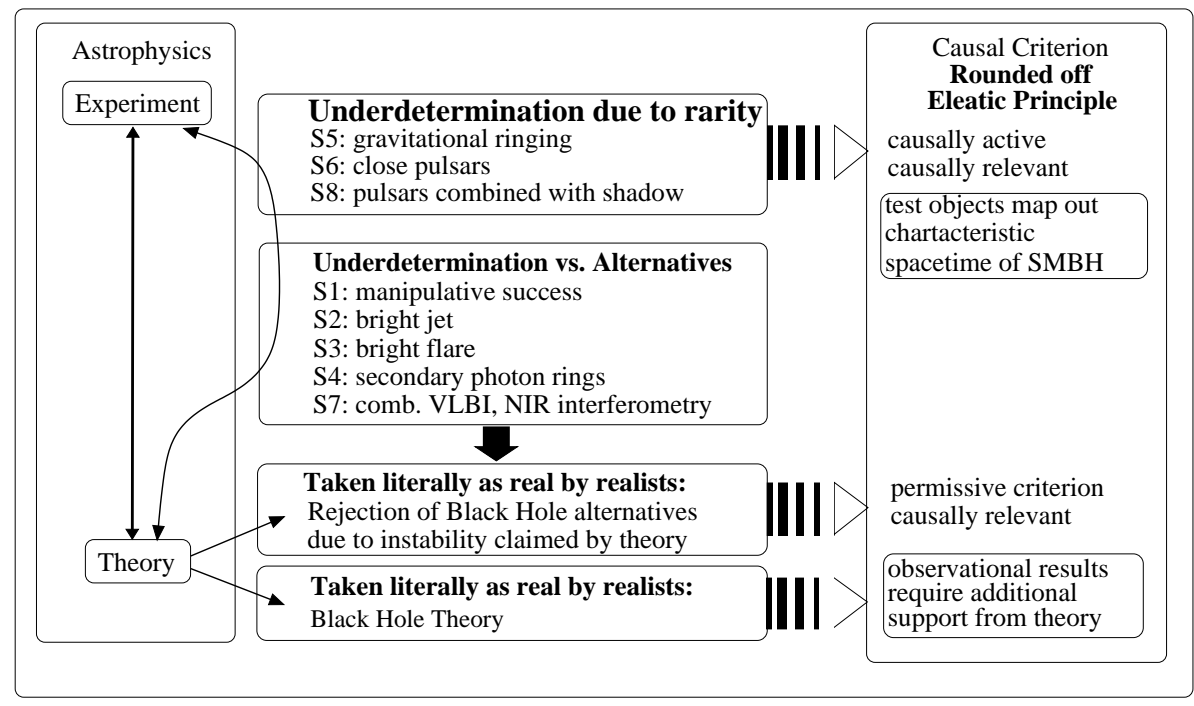

Fig. 13 Linkage between experiment and theory interpreted via the concept of realism, underdetermination and a "rounded out" version of the Eleatic Principle, here shown with respect to the results of our investigation. For comparison see also Fig 1 which we adopted here for the case of the Galactic Center SMBH.

flow chart for option $S_{8}$ to combine suitable necessary conditions. Details of the chart are described in option $S_{7}$ as well.

\section{Summary and Conclusion}

It appears that acceptance and proof of the presence of a SMBH is in a state quite comparable to e.g. the acceptance of the existence of atoms and molecules at the beginning of the past century. The Eleatic Principle as a heuristic causal criterion to probe the existence of an entity has been very helpful in that process. A detailed analysis of how the necessary conditions need to be combined to a convincing case will also be essential in the case of the SMBHs.

A challenge for astrophysics can be summarized in the question: How clean are the observational cases that may serve as logical entities for the causal criterion test? How rare are the "smoking gun" cases and the coincidence of fully operational, complex experiments and a "cooperative" source, such that the expected or predicted phenomena do occur and can actually be measured. The "manipulative success" approach $\left(S_{1}\right)$ may be contaminated by statistical noise as one needs a finite number of objects of which SgrA* will only be one. The success of the method will certainly also depend on the choice of the observables on which statistics is done. Occasional bright jets or flares $\left(S_{2}\right.$ and $\left.S_{3}\right)$ are probably very rare, but are likely to have occurred during the past of $\operatorname{Sgr} A^{*}$ (see estimates and references above). In these cases the cleanness of the observables will depend on the physical properties of the jet (e.g. its orientation to the line of sight) or the brightness and duration of the flare. Similarly gravitational wave ringing $\left(S_{5}\right)$ characteristic for interactions of large masses may be rare or very difficult to measure. The detection of a secondary photon ring $\left(S_{4}\right)$ will only be possible if the accretion process is well ordered and not too violent such that high contrast observations of the vicinity of the SMBH can be carried out successfully.

A conceptual or philosophical challenge arises from the usage of a two stage approach based on the concept of "(Anti)Realism and Underdetermination" and a 
modified form of the Eleatic Principle (section 3.1, B, C). This approach is mainly driven by the theoretical and observational necessity that forces us to deal with the observational consequences imposed by theory (e.g. the fact that it takes an infinite amount of time to reach the event horizon) and the deficiencies in the measuring process. There is hope that the latter can be minimized by instrumental and observational progress and an elegant and fruitful combination of different methods to approach the observational problems. The application of these concepts then may give us enough confidence to accept the validity or reality of the black hole theory - either literally or based on the observational results that point at the reality of (in our case) supermassive black holes. Trust in theory and observational results may then lead us to involve causation as a clue to the existence of supermassive blck holes in the way it is laid out in section and Appendix $\mathrm{C}$ and discussed in detail in, e.g., Colyvan (1998) and Marcus (2015). With respect to the results of our investigation we have depicted the situation in Fig 13. As shown in this figure, our results are successfully mapped out by this combination of concepts.

Underdetermination arises both from the rarity of the expected event and from the fact that the observations alone can probably not clearly discriminate the black hole case against possible alternatives. Here, only the theoretical finding can help, that alternatives do not lead to stable solution. As in the case of a standpoint that a pure realist may take concerning black hole theory, such a reasoning then requires a permissive criterion as proposed by Sellars (1963) and Psillos (2011a). This leads to applying the "rounded out" version of the Eleatic Principle described by Colyvan in 1998 (Colyvan, 1998). However, even though in the end one may need additional support through theoretical concepts, it needs to be stressed that here we are not concerned with the question of the ontological state of the theoretical concept but rather with maximizing the acceptance of an identification as a SMBH through the comparison to the theoretical concept of it to test the properties of an observable entity against a theoretical concept in order to identify it in a commonly agreable way with the object described by this very theoretical concept (see paragraph "Theory taken as being real" in section B). A further challenge arises from the complexity of the observables and the corresponding quality of the necessary and sufficient condition 26 .

Several of the sufficient conditions we outlined in section 7 are more biased towards the mere detection of a very high mass concentration than the distinction between an SMBH and one or several of the discussed alternative explanations. With evermore complex conceptual models for compact masses that may serve as

26 In the context of the SMBH candidate $\mathrm{SgrA}^{*}$ at the Galactic Center and the large number of achievable and potential observables, the conceptual strategy laid out in section 3 and depicted in Fig. 3 is not trivial. If several combinations of necessary conditions may lead to a convincing statement concerning the existence of an $\mathrm{SMBH}$ at the center of the Milky Way, then a conceptual or philosophical challenge arises through the fact that some are more convincing than others. A mere measurement of a relativistic jet might be less valued than the detection of an pulsar extremely close to the black hole. In this case, we would have to refrain from Boolean logic, in which only true and false ( 1 or 0 ) are allowed as values of the necessary and sufficient conditions $N$ and $S$ and the resulting value $K$ (see section 3.3 ). A many-valued logic in which the values of these conditions can be any real number between 0 and 1 is then a possible way and equations (5) to (10) must be modified accordingly.

Using the notation from section 3.3 equation (8) would then for the sufficient condition $\lambda^{\prime}$ translate into:

$$
K_{\lambda^{\prime}}=\Pi_{i=1}^{\mu\left(\lambda^{\prime}\right)} \quad N_{\kappa_{i, \mu\left(\lambda^{\prime}\right)}}
$$

The value of $\mathrm{K}$ will be high in case that a combination of necessary conditions have been met well. Then the favorite sufficient condition would have a value of:

$$
K=\max \left[K_{\lambda} \mid \lambda=1, \nu\right]=\max \left[\Pi_{i=1}^{\mu(\lambda)} \quad N_{\kappa_{i, \mu(\lambda)}} \mid \lambda=1, \nu\right] .
$$


an alternative to a SMBH it becomes also more difficult to judge if one falls into some of the pit holes presented on the right panel in Fig. 3. Do we oversee necessary conditions $(\eta)$ ?; Are we combining the rights ones (e.g. $\epsilon$ or $\eta)$ ?; etc.

High chances of success for delivering convincing results concerning the achievement of technical success and the repeatability of the experiments are represented by the combination of millimeter and infrared interferometry in possible combination with the pulsar measurements $\left(S_{6}, S_{7}\right.$ and $\left.S_{8}\right)$. If GRAVITY finds stars closer in - or even with the current availability of known high velocity S-stars - GRAVITY may be the only experiment that allows us to determine the location of the SgrA* emission with respect to the central mass position, i.e. the object about which the stars are in orbit. A (temporarily) bright accretion disk may be too turbulent and too much effected by optical depth and light bending effects. Hence the usefulness of determining the location of the mass center may be too much model dependent (compared to the determination of a stellar orbit). Images of the material close to the SgrA* supermassive black hole will be shortly obtained by the EHT.

If detailed mapping of the spacetime is not possible due to the lack of suitable pulsars or stars, it appears that the only argument that allows us to easily reject the boson star (or other alternative models) possibility is the large number of additional requirements (e.g. the existence of special particles) that need to be fulfilled to sustain its existence, in particular the fact that it is very fragile and - if stability conditions are disturbed - it will collapse into a black hole.

Hence, it appears that $\mathrm{SgrA}^{*}$ is indeed already an outstanding case to prove the existence of SMBHs. Further support for the existence of supermassive black holes in general may come from gravitational wave experiments like eLISA. However, due to its proximity, SgrA* highest angular resolution observations translate into high linear resolution at the location of $\mathrm{SgrA}^{*}$. This results in a large number of necessary conditions that can be probed and fulfilled (see Tab 3). New instruments will allow for new high precession measurements in the very near future that are certain to improve the quality of $\mathrm{SgrA}^{*}$ as a convincing showcase for a SMBH that can be "accepted as real". However, it appears that the experiments or observational effects that may come up with the most convincing arguments for the existence of supermassive black holes are also the ones that are the most difficult to achieve or the rares to happen. Yet, despite all the great efforts put forward by researchers, it is necessary that $\mathrm{SgrA}^{*}$ and its immediate surroundings provide us with useful flares as well as stars and pulsars with suitable orbits for maximizing the usefulness of the measurements.

\section{Acknowledgments}

We thank Sybille Anderl (IPAG Grenoble) for valuable comments and support on the philosophy of science sections, Georgi Dvali (LMU Munich) for constructive and valuable discussions and input, and Grischa Karssen (University of Cologne) for contributing Fig 12 and part of the corresponding discussion. This work was supported in part by the Deutsche Forschungsgemeinschaft (DFG) via the Cologne Bonn Graduate School (BCGS), the Max Planck Society through the International Max Planck Research School (IMPRS) for Astronomy and Astrophysics. Part of this work was supported by fruitful discussions with members of the European Union funded COST Action MP0905: Black Holes in a Violent Universe and the Czech Science Foundation DFG collaboration (No. 14-37086G) and with members of the European Union Seventh Framework Program (FP7/2007-2013) under grant agreement No. 312789, Strong Gravity: Probing Strong Gravity by Black Holes Across the Range of Masses. 


\section{A An example for establishing existence claims}

As an example for establishing existence claims in the (recent) history of physics, we look at the case of atoms and molecules. The first case we want to look at - because it has been widely discussed in philosophy of science - is the final acceptance of the existence of molecules/atoms due to the work of Jean Perrin on Brownian motion in the early 20th century as the collision with the quick atoms or molecules in the gas or liquid. Brownian motion itself was first discovered by the Scottish botanist Robert Brown in 1827 as random motion of particles.

Perrin received the Nobel Prize for physics in 1926 for his work that "put a definite end to the long struggle regarding the real existence of molecules" (C.W. Oseen, member of the Nobel Committee for Physics). So, how was Perrin able to establish the existence of molecules/atoms and what can we learn from this for the question of the existence of black holes? Atoms and molecules among other places played a major role in the kinetic theory of heat. However, there were debates about whether atoms/molecules were merely a useful fiction that yielded (in many cases) the right result or whether they should be accepted as real. For some time it appeared to be the case that this question could not be resolved by experimental means. In the early 20th century (partly through the work of Einstein) atoms and molecules became accessible, i.e. new theoretical means were devised so as to bring the hypothesis into the reach of established experimental methods (see Psillos, 2011b, "Making Contact with Molecules: On Perrin and Achinstein" ).

In making the question of atoms/molecules not only experimentally accessible but to decide it positively, three features proved to be important:

i. The assumption that atoms/molecules exist puts constraints on observational data: The assumptions could only be true if some observable magnitudes had certain very definite values (Avogadro's number/constant). This appears to be an essential point in a number of cases. What is asked for here are novel predictions, i.e. the prediction of phenomena, which would be very unlikely if atoms/molecules did not exist (compare the prediction of the deflection of light by the sun as evidence for GR; Karl Popper tried to capture this by his notion of a high degree of falsifiability). The prediction has to be risky.

ii. On rival accounts, i.e. on the denial of atoms/molecules, the value of Avogadro's number would have been completely different. Hence, the value of Avogadro's constant is dependent on the existence of atoms.

iii. The relevant value was experimentally established in a number of different/independent ways. Avogadro's constant is tested in several different ways and today there are about 60 different and independent ways to measure it (Bethge et al, 2012), making use of e.g. electrical charge and current, in determinating the properties of gas, liquids and crystals.

Following the ground breaking work of Perrin, today the acceptance of atoms is supported by direct imaging of molecules, atoms or even their bounds (e.g. de Otevza et al, 2013; Iwata et al, 2015) 27.

\section{B From (Anti)realism and Underdetermination to a Causal Principle}

How for theory and observations the concepts of "Realism" and "Underdetermination" may be linked to causation is outlined in the following:

Theory taken literally as being true: Black holes are extremely simple objects and hence, ideally suited to be regarded as a kind of theoretical concept that historically has the tendency to be taken literally as real by realists (see below and statements by Stathis Psillos and Wilfrid Sellars (Psillos, 2011a; Sellars, 1963)). The problem is that other theories could be regarded as being similarly simple (although they are often more complex as they require the definition of additional quantities like particle masses and interaction forces etc.). The fact that one cannot easily distinguish between them experimentally, leaves enough freedom to being unable to decide if some other entities implied by a literal reading of the theory, e.g. a compact object like a black hole and its possible alternatives, are indeed real (e.g. the shadow of such a compact object, i.e. the presence, depth and size of an emission free or sparse region close to the position of the compact object; see section 4.10).

Here, also the possibility of an experimental comparison between theory and implied entities (if interpreted as predicted phenomena like speeds of stars or emitting blobs, timescales associated with consecutive events, or structures like the shadow) in a way relieves many philosophers (especially if facts or statements are being taken literally) from the "commitment to a host of entities with a (to say the least) questionable ontic status: numbers, geometrical points, theoretical ideals, models and suchlike" (Psillos, 2011a).

27 The case of pulsars and the case of the identification of sources in which gamma-ray bursts originate may be a further example in which establishing existence claims is or has been important in the the recent history of physics. 
Therefore, we must clarify that at this point we are not concerned with the question of whether there is reality to the theoretical concept of a black hole as such, for instance as a concept within the theory or relativity. The goal of the observational and experimental astrophysics is to test the properties of an observable entity against a theoretical concept in order to identify it in a commonly agreeable way with the object described by this very theoretical concept.

Reality of entities derived from theory: The entities implied by the theory of black holes (here taken as observational results or as implied physical scenarii, i.e. how stars orbit the black hole or - in the near future - how exactly the black hole shadow looks like) can also be regarded as real, as they all come (are derived) indeed "from theory and ... there is no theory-free standpoint from which" (Psillos, 2011a), these entities can be viewed. This means, as valuable observational predictions are usually all derived from theory there is no theory-independent criterion of reality that can be used in an accepted way. The goal is to define and successfully observed entities i.e. predictions, for which a black hole plays an indispensable role in their explanation.

While this is clearly the preferred situation for the physicist (i.e. a successful comparison between observational results or the properties of implied physical scenarii) this is also true for theory as such from the viewing angle of realists that historically tend to read (take) theories literally as real. As this can be explained out of theory it can be regarded as a permissive criterion (Sellars, 1963) that particularly does not disallow abstract entities from being real. Stathis Psillos (Psillos, 2011a) points out that "this explanatory criterion should not be confused with a causal criterion", e.g. in the form of the Eleatic Principle (see below), "according to which being causally active, that is having causal powers, is a criterion of objecthood. Causal efficacy may well be a mark of reality, but not everything that is real is causally efficacious" (Psillos, 2011a). The former would be good for experimentalists, the latter would be supported by black hole theory. Here, Psillos points at entities that are causally idle but causally relevant. Hence, he describes Colyvan's "rounded out" version of the Eleatic Principle (Colyvan 1998 (Colvvan, 1998) and the following section (C).

Causation as a clue to probe existence: For observed entities, may they be derived from theory or not, causation may be taken as a clue to make their existence plausible beyond doubt. David M. Amstrong states in his reply to Reinhardt Grossmann in the correspondence on the "Ontology and the Physical Universe" (Cumpa and Tegtmeier, 2009) that if one does not "postulate an entity [...] which is not required in ones account of causation", then indeed causation can be used as a partial clue to the existence. The word "partial" is used here as it needs to be seen how the analysis of the causation needs to be done in every particular case. Goal of the observational astrophysics is to optimize this aspect.

As Andrew Newman states: "Talking about existing and talking about being real are just different ways of talking about the same thing" Newmann (2002). As supporting examples he quotes Gottlob Frege and Bertrand Russell who are guided to things that are real by syntax (e.g. in Carnap, 1968), W.V. Quine, who demands that something must be quantifiable if it is called real (e.g. in Deccock and Horsten, 2000), and D.M. Amstrong demanding causal significance for real things (e.g. in Newmann, 2002). Causal significance or relevance is an essence of any form of the Eleatic Principle (see below).

Andrew Newman points out (Newmann, 2002): "Alex Oliver claims that the Eleatic Principle is ambiguous because there is an epistemological reading of 'reason' and a metaphysical reading of 'reason', mostly worried about the existence of causally inactive entities." This criticism might appear to be applicable to aspects of the black hole physics, however, all experiments aim at consolidating causal activity.

Reality and the Eleatic Principle: In the case of black holes, we may make use of the possible claim that the physical world is causally closed, i.e. that all genuine causes of physical events are physical causes (e.g. Montero, 2003). Hence, in the case of black holes we do not run into the problem that this particular science entity is not reducible to a physical entity. Even if the reduction to such an entity is currently not fully conducted, all instrumental and observational efforts aim at improving the performance of that reduction.

\section{The Eleatic Principle}

If underdetermination can be fought or even partially overcome, then causation may be used to further underline the realism or existence of an entity in a generally acceptable way. This involves the usage of a causal criterion that may be in the form of the Eleatic Principle (for a general overview see e.g. Colyvan, 1998, 2001). (Colyvan, 1998) gives a consice definition of the classical Eleatic Principle "An entity is to be counted as real if and only if it is capable of participating in causal processes".

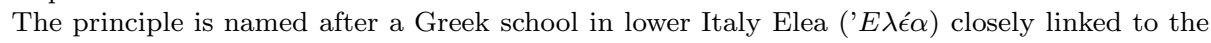
philosophers Parmenides, Zeno and Xenophanes of Colophon (going back to a figure in Plato's dialogue Sophistes). As a philosophical conceptual aspect, the School of Elea rejects any epistemological criteria simply based on sensual experiences (Fig 21). According to the Eleatic Principle, we should be realists about whatever manifests itself in virtue of having effects (in 'A World of States 
of Affairs' by David M. Amstrong (Armstrong, 2001), see also the discussion in "Quining Naturalism" by Huw Price (Price, 2007)). The use of the Eleatic Principle in our context needs some clarification. The classical version of the Eleatic Principle uses and connects (in a more time-like fashion) mainly causally active entities. In this version non-causal entities like abstract mathematical and physical concepts are regarded as causally idle and therefore they are not considered as suitable entities. This problem has been explained in detail by Mark Colyvan in his article "Can the Eleatic Principle be Justified?" (Colvvan, 1998). He investigates several justification attempts and shows how and when they fail or what their drawbacks are. In order to avoid these problems he suggests to use a "rounded out" version of the Eleatic Principle in which also causally idle entities can be used. This "rounded out" version contains the classical version of the Eleatic Principle (Fig 2). In the case of the Galactic Center and supermassive black holes in general, involvement of at least the "rounded out" version of the Eleatic Principle is justified (see below and Colvvan, 1998).

Mark Colyvan's version of the "rounded out" Eleatic Principle does not stand alone. He points out that this more general principle that he proposes has great similarities to Quine's thesis that "we are only ontologically committed to all and only the entities that are indispensable to our current best scientific theories" (see Quine, 1948). Indispensable entities then include both causally idle and causally efficacious entities. A critical discussion of the Eleatic Principle with a focus on the role of mathematical objects and Colyvan's defense of Quine's indispensability argument is given by Marcus (2015). It should also be mentioned that the entities we connect in the synthesis section 7 have by themselves a rather complex nature. In most cases they are the result of a logical combination of causally idle and active entities and have a structure similar to that shown in equation (9). Causally idle (but ally relevant) entities occur e.g. through the theory of image formation in all wavelength domains or in relativistic concepts that are used to extract expected situations and compare them to measurements. In particular the "data inversion" problem in the image formation theory of interferometry might well serve as an example of true "underdetermination" in astrophysics. Causally efficacious entities occur e.g. in the form of stars, pulsars, emitted radiation or astrophysical instruments and telescopes, all of which can be located in spacetime in contrast to the causally idle entities.

\section{Time scales and luminosities of gravitational wave "ringing"}

The analysis is done based on the formalism presented in Misner et al (1973) and Shapiro and Teukolskv (1983). The tidal disruption radius $r_{\mathrm{T}}$ for main-sequence stars with mass $m_{\star}$ and radius $R_{\star}$ is larger than the Schwarzschild radius $R_{\mathrm{S}}$ of the supermassive black hole,

$$
r_{\mathrm{T}} \approx 19 R_{\mathrm{S}}\left(\frac{R_{\star}}{R_{\odot}}\right)\left(\frac{M_{\bullet}}{4 \times 10^{6} M_{\odot}}\right)^{1 / 3}\left(\frac{m_{\text {star }}}{1 M_{\odot}}\right)^{-1 / 3}
$$

so we cannot expect any gravitational wave event from main-sequence star inspirals, since they will be tidally disrupted. On the other hand, Schwarzschild radius is larger than tidal disruption radius for white dwarfs with $R_{\star}=0.01 R_{\odot}$, neutron stars with $R_{\star}=10^{-5} R_{\odot}$, and stellar black holes, so these are not subject to tidal disruption.

A circularized orbit of a compact star with the semimajor axis $a_{\star}$ around the supermassive black hole $M \bullet$ emits gravitational waves at frequency,

$$
\nu_{\mathrm{GW}}=\frac{2}{P_{\mathrm{orb}}}=0.18 \mathrm{mHz}\left(\frac{\mathrm{M}_{\bullet}}{4 \times 10^{6} \mathrm{M}_{\odot}}\right)^{1 / 2}\left(\frac{\mathrm{a}_{\star}}{10 \mathrm{R}_{\mathrm{S}}}\right)^{-3 / 2}
$$

reaching $\sim 1.1 \mathrm{mHz}$ at innermost stable circular orbit. is

The characteristic scaling amplitude for the EMRI event at the distance of the Galactic center

$$
h_{0}=4 \times 10^{-18}\left(\frac{M_{\bullet}}{4 \times 10^{6} M_{\odot}}\right)^{2 / 3}\left(\frac{m}{1 M_{\odot}}\right)\left(\frac{D}{8 \mathrm{kpc}}\right)^{-1}\left(\frac{\nu_{\mathrm{GW}}}{1 \mathrm{mHz}}\right)^{2 / 3},
$$

and the effective metric perturbation is given by the amplitude $h_{0}$ times the square root of the number of cycles the EMRI spends in band with a bandwidth $\Delta \nu$ centered at the frequency $\nu$. The number of cycles $N$ is given by $N=\nu^{2} / \dot{\nu}=\nu \tau$, where the characteristic evolution time-scale $\tau \equiv \nu / \dot{\nu}=8 / 3\left(t_{\text {coal }}-t\right) \propto \mathcal{M}^{-5 / 3} \nu^{-8 / 3}$ and $t_{\text {coal }}$ is the coalescence time. The 'chirp mass' may be expressed as $\mathcal{M}=\left(M_{\bullet} m_{\star}\right)^{3 / 5} /\left(M_{\bullet}+m_{\star}\right)^{1 / 5} \approx M_{\bullet}^{2 / 5} m_{\star}^{3 / 5}$, where the approximation holds for the EMRI. Finally, the metric perturbation or basically the signal is proportional to $S \propto h_{0}(\mathcal{M} \nu)^{-5 / 6}=h_{0} M_{\bullet}^{-1 / 3} m_{\star}^{-1 / 2} \nu^{-5 / 6}$.

The expected luminosity $L_{\mathrm{GW}}$ averaged over one period and evaluated at the innermost stable circular orbit (Peters and Mathews, 1963) is 


$$
L_{\mathrm{GW}}=1.9 \times 10^{43} \mathrm{erg} \mathrm{s}^{-1}\left(\frac{\mathrm{m}_{\star}}{1 \mathrm{M}_{\odot}}\right)^{2}\left(\frac{\mathrm{M}_{\bullet}}{4 \times 10^{6} \mathrm{M}_{\odot}}\right)^{3}\left(\frac{\mathrm{a}_{\star}}{3 \mathrm{R}_{\mathrm{S}}}\right)^{-5},
$$

which is valid for circular orbits. In general, the equation 23 depends strongly on the orbital eccentricity (Peters and Mathews, 1963). The incoming flux $F_{\mathrm{GW}}$ may be expressed as a function of the amplitude $h_{0}$ and the observed frequency $\nu_{\mathrm{GW}}$,

$$
F_{\mathrm{GW}} \simeq 0.005\left(\frac{\nu_{\mathrm{GW}}}{1 \mathrm{mHz}}\right)^{2}\left(\frac{h_{0}}{4 \times 10^{-18}}\right)^{2} \mathrm{erg} \mathrm{cm}^{-2} \mathrm{~s}^{-1} .
$$

The coalescence (merger) time-scale $\tau_{\text {merge }}$ depends on eccentricity of the orbit $e$. For a circular orbit,

$$
\tau_{0}\left(a_{0}\right) \approx 7800 \mathrm{yr}\left(\frac{\mathrm{M}_{\bullet}}{4 \times 10^{6} \mathrm{M}_{\odot}}\right)^{-2}\left(\frac{\mathrm{m}_{\star}}{1 \mathrm{M}_{\odot}}\right)^{-1}\left(\frac{\mathrm{a}_{0}}{10 \mathrm{R}_{\mathrm{S}}}\right)^{4},
$$

where $a_{0}$ is an initial semi-major axis. For initially highly eccentric orbits, $e \approx 0.99$, it reduces to $\tau_{\text {merge }}=768 / 425 \tau_{0}\left(a_{0}\right)\left(1-e_{0}\right)^{7 / 2} \approx 0.016 \mathrm{yr}$.

The detection of an EMRI event associated with the Galactic center black hole would enable to precisely measure the mass and the spin of the black hole with an independent measurement. The waveform could also reveal deviations from the Kerr metric as well as a possible different character of a central compact object (e.g. a boson star). An exemplary waveform for an EMRI event for the ratio $m_{\star} / M_{\bullet}=10^{-4}$ and the black hole spin $J=0.998$ is depicted in Fig. 9 The inspiral starts at four gravitational radii.

The EMRI events from the Galactic center will be within the detection sensitivity limits of the planned LISA and eLISA space-base interferometers that will be capable to detect the gravitational wave events with low frequencies in the range $\nu_{\mathrm{GW}}=0.1 \mathrm{mHz}-1 \mathrm{mHz}$. Although the likelihood to detect an EMRI event for the Galactic center is rather low during the mission lifetime, Gair et al (2004) estimate that LISA will be able to detect $\sim 2$ EMRI events of $1.4 M_{\odot}$ compact objects (white dwarfs and neutron stars) per cubic Gpc per year for black holes with similar masses as Sgr A*. 


\section{References}

Aasi J, Abadie J, Abbott BP, Abbott R, Abbott T, Abernathy MR, Accadia T, Acernese F, Adams C, Adams T, et al (2013) Directed search for continuous gravitational waves from the Galactic center. Phys. Rev. D88(10):102002, 1309.6221

Aasi J, Abbott BP, Abbott R, Abbott T, Abernathy MR, Acernese F, Ackley K, Adams C, Adams $\mathrm{T}$, Addesso P, et al (2014) Search for gravitational wave ringdowns from perturbed intermediate mass black holes in LIGO-Virgo data from 2005-2010. Phys. Rev. D89(10):102006, 1403.5306

Abbott BP, Abbott R, Abbott TD, Abernathy MR, Acernese F, Ackley Kea (2016) Observation of gravitational waves from a binary black hole merger. Phys Rev Lett 116:061,102, URL http://link.aps.org/doi/10.1103/PhysRevLett.116.061102

Abdolrahimi S, Mann RB, Tzounis C (2015) Distorted local shadows. Phys. Rev. D91(8):084052

Abramowicz MA, Kluźniak W, Lasota JP (2002) No observational proof of the black-hole eventhorizon. A\&A396:L31-L34, astro-ph/0207270

Amaro-Seoane P, Gair JR, Freitag M, Miller MC, Mandel I, Cutler CJ, Babak S (2007) TOPICAL REVIEW: Intermediate and extreme mass-ratio inspirals astrophysics, science applications and detection using LISA. Classical and Quantum Gravity 24:R113-R169

Amaro-Seoane P, Aoudia S, Babak S, et al PB (2012) Low-frequency gravitational-wave science with elisa/ngo. Classical and Quantum Gravity 29(12):124,016

Anderl S (2015) Astronomy and Astrophysics in the Philosophy of Science. ArXiv e-prints 1510.03284

Anderl S (2016) Astronomy and Astrophysics. The Oxford Handbook of Philosophy of Science Edited by Paul Humphreys DOI DOI:10.1093/oxfordhb/9780199368815.013.45

Arca-Sedda M (2016) On the formation of compact, massive subsystems in stellar clusters and its relation with intermediate-mass black holes. MNRAS455:35-50, 1502.01242

Armstrong D (ed) (2001) A World of States of Affairs; Cambridge: Cambridge University Press, p.41; (ISBN-13: 9780521580649 - ISBN-10: 0521580641)

Baganoff FK, Bautz MW, Brandt WN, Chartas G, Feigelson ED, Garmire GP, Maeda Y, Morris M, Ricker GR, Townsley LK, Walter F (2001) Rapid X-ray flaring from the direction of the supermassive black hole at the Galactic Centre. Nature413:45-48

Baganoff FK, Maeda Y, Morris M, Bautz MW, Brandt WN, Cui W, Doty JP, Feigelson ED, Garmire GP, Pravdo SH, Ricker GR, Townsley LK (2003) Chandra X-Ray Spectroscopic Imaging of Sagittarius A* and the Central Parsec of the Galaxy. ApJ591:891-915

Bahcall JN, Wolf RA (1976) Star distribution around a massive black hole in a globular cluster. ApJ209:214-232

Balick B, Brown RL (1974) Intense sub-arcsecond structure in the galactic center. ApJ194:265-270

Barbour JB, Pfister H (eds) (1995) Mach's Principle: From Newton's Bucket to Quantum Gravity. Birkhäuser, ISBN 978-0-8176-3823-8

Bardeen JM (1973) Rapidly rotating stars, disks, and black holes. In: DeWitt C, DeWitt BS (eds) Black Holes (Les Astres Occlus), pp 241-289

Bardeen JM, Wagoner RV (1971) Relativistic Disks. I. Uniform Rotation. ApJ167:359

Barrau A, Rovelli C, Vidotto F (2014) Fast radio bursts and white hole signals. Phys. Rev. D90(12):127503

Barrau A, Bolliet B, Schutten M, Vidotto F (2016) Bouncing black holes in quantum gravity and the Fermi gamma-ray excess. ArXiv e-prints 1606.08031

Barrière NM, Tomsick JA, Baganoff FK, Boggs SE, Christensen FE, Craig WW, Dexter J, Grefenstette B, Hailey CJ, Harrison FA, Madsen KK, Mori K, Stern D, Zhang WW, Zhang S, Zoglauer A (2014) NuSTAR Detection of High-energy X-Ray Emission and Rapid Variability from Sagittarius A* Flares. ApJ786:46, 1403.0900

Bartusiak M (2015) Black hole; Yale University Press, New Haven \& London, ISBN: 9780300210859

Berti E, Barausse E, Cardoso V, Gualtieri L, Pani P, Sperhake U, Stein LC, Wex N, Yagi K, Baker T, Burgess CP, Coelho FS, Doneva D, De Felice A, Ferreira PG, Freire PCC, Healy J, Herdeiro C, Horbatsch M, Kleihaus B, Klein A, Kokkotas K, Kunz J, Laguna P, Lang RN, Li TGF, Littenberg T, Matas A, Mirshekari S, Okawa H, Radu E, O'Shaughnessy R, Sathyaprakash BS, Van Den Broeck C, Winther HA, Witek H, Emad Aghili M, Alsing J, Bolen B, Bombelli L, Caudill S, Chen L, Degollado JC, Fujita R, Gao C, Gerosa D, Kamali S, Silva HO, Rosa JG, Sadeghian L, Sampaio M, Sotani H, Zilhao M (2015) Testing general relativity with present and future astrophysical observations. Classical and Quantum Gravity 32(24):243001

Berti E, Sesana A, Barausse E, Cardoso V, Belczynski K (2016) Spectroscopy of Kerr Black Holes with Earth- and Space-Based Interferometers. Physical Review Letters 117(10):101102

Bethge K, Gruber G, Stöhlker T (eds) (2012) Physik der Atome und Moleküle: Eine Einfhrung; John Wiley \& Sons, 14. Mai 2012, ISBN 978-3-527-66255-5, p.4445;

Bilic N, Tupper GB, Viollier RD (2003) Supermassive fermion balls and constraints from stellar dynamics near Sgr A*. ArXiv Astrophysics e-prints astro-ph/0310172

Bird S, Cholis I, Muñoz JB, Ali-Haïmoud Y, Kamionkowski M, Kovetz ED, Raccanelli A, Riess AG (2016) Did LIGO detect dark matter? ArXiv e-prints 1603.00464 
Blais D, Kiefer C, Polarski D (2002) Can primordial black holes be a significant part of dark matter? Physics Letters B 535:11-16, astro-ph/0203520

Blandford RD, Begelman MC (1999) On the fate of gas accreting at a low rate on to a black hole. MNRAS303:L1-L5, astro-ph/9809083

Boehle A, Ghez AM, Schödel R, Meyer L, Yelda S, Albers S, Martinez GD, Becklin EE, Do T, Lu JR, Matthews K, Morris MR, Sitarski B, Witzel G (2016) An Improved Distance and Mass Estimate for Sgr A* from a Multistar Orbit Analysis. ApJ830:17, 1607.05726

Bogdanović T (2015) Supermassive Black Hole Binaries: The Search Continues. Astrophysics and Space Science Proceedings 40:103, 1406.5193

Branham RL (2014) The distance to the Galactic center determined by OB stars. Ap\&SS353:179190

Britzen S, Eckart A, Lämmerzahl C, et al JR (2015) Jet signatures of black holes: From Sgr A* to active galactic nuclei. In: Astronomische Nachrichten, Vol. 5/2015, page 471-476

Broderick AE, Loeb A (2005) Imaging bright-spots in the accretion flow near the black hole horizon of Sgr A*. MNRAS363:353-362, astro-ph/0506433

Broderick AE, Loeb A (2006) Imaging optically-thin hotspots near the black hole horizon of Sgr $\mathrm{A}^{*}$ at radio and near-infrared wavelengths. MNRAS367:905-916, astro-ph/0509237

Broderick AE, Narayan R (2007) Where are all the gravastars? Limits upon the gravastar model from accreting black holes. Classical and Quantum Gravity 24:659-666, gr-qc/0701154

Broderick AE, Johannsen T, Loeb A, Psaltis D (2014) Testing the No-hair Theorem with Event Horizon Telescope Observations of Sagittarius A*. ApJ784:7

Broderick AE, Narayan R, Kormendy J, Perlman ES, Rieke MJ, Doeleman SS (2015) The Event Horizon of M87. ApJ805:179

Brown E (2010) Ann E. Ewing, 89, Washington Post, Sunday, August 1, 2010, page 39, http://www.washingtonpost.com/ wp-dyn/content/article/

Buchholz RM, Schödel R, Eckart A (2009) Composition of the galactic center star cluster. Population analysis from adaptive optics narrow band spectral energy distributions. A\&A499:483-501, 0903.2135

Bursa M, Abramowicz MA, Karas V, Kluźniak W, Schwarzenberg-Czerny A (2007) The timescale of encircling light. In: Hledík S, Stuchlík Z (eds) Proceedings of RAGtime 8/9: Workshops on Black Holes and Neutron Stars, pp 21-25

Calmet X (2015) Quantum Aspects of Black Holes; ISBN 978-3-319-10852-0. (ed), Springer, Heidelberg

Cardoso V, Crispino LCB, Macedo CFB, Okawa H, Pani P (2014) Light rings as observational evidence for event horizons: Long-lived modes, ergoregions and nonlinear instabilities of ultracompact objects. Phys. Rev. D90(4):044069

Cardoso V, Franzin E, Pani P (2016) Is the Gravitational-Wave Ringdown a Probe of the Event Horizon? Phys Rev Lett 116, 171101116

Carnap R (1968) Logische Syntax der Sprache, 1968, ISBN 978-3-662-25376-2(e-book), ISBN 9783-662-23331-3; 1934 by Julius Springer, 2. Auflage

Chakravartty A (2010) Anjan Chakravartty. A Metaphysics for Scientific Realism: Knowing the Unobservable - 978-0-521-13009-7; 2015 Paperback: ISBN-10: 1508932867, ISBN-13: 9781508932864

Chandrasekhar S (1983) The mathematical theory of black holes, Oxford University Press

Chapline G (2003) Quantum Phase Transitions and the Failure of Classical General Relativity. International Journal of Modern Physics A 18:3587-3590, gr-qc/0012094

Chatzopoulos S, Fritz TK, Gerhard O, Gillessen S, Wegg C, Genzel R, Pfuhl O (2015) The old nuclear star cluster in the Milky Way: dynamics, mass, statistical parallax, and black hole mass. MNRAS447:948-968, DOI 10.1093/mnras/stu2452, 1403.5266

Christodoulou M, Rovelli C, Speziale S, Vilensky I (2016) Planck star tunneling time: An astrophysically relevant observable from background-free quantum gravity. Phys. Rev. D94(8):084035

Clavel M, Terrier R, Goldwurm A, Morris MR, Ponti G, Soldi S, Trap G (2013) Echoes of multiple outbursts of Sagittarius A revealed by Chandra. A\&A558:A32, 1307.3954

Cleland C (2002) Living with the abstract: realism and models. Philosophy of Science 69:474-496

Colbert EJM, Mushotzky RF (1999) The Nature of Accreting Black Holes in Nearby Galaxy Nuclei. ApJ519:89-107, astro-ph/9901023.

Colpi M, Shapiro SL, Wasserman I (1986) Boson stars - Gravitational equilibria of self-interacting scalar fields. Physical Review Letters 57:2485-2488

Colyvan M (1998) Can the Eleatic Principle be Justified? Canadian Journal of Philosophy 28:313335

Colyvan M (2001) The Indispensability of Mathematics; Oxford University Press, 22.03.2001; ISBN $0-19-513754-\mathrm{X}$

Contini M (2011) The low-luminosity active galactic nucleus in the centre of the Galaxy. MNRAS418:1935-1947, 1104.1282

Cumpa J, Tegtmeier E (2009) Phenomenological Realism Versus Scientific Realism; Cumpa, J. \& Tegtmeier, E. (Eds.); Metaphysical Correspondences; 2009 Ontos Verlag; P.O.Box 1541, D63133 Heusenstamm; ISBN 978-3-86838-051-4; 
Cunha PVP, Herdeiro CAR, Radu E, Rúnarsson HF (2015) Shadows of Kerr Black Holes with Scalar Hair. Physical Review Letters 115(21):211102, 1509.00021

Danzmann K (2015) eLISA and the Gravitational Universe. IAU General Assembly 22:2248153

De Paolis F, Ingrosso G, Nucita AA, Orlando D, Capozziello S, Iovane G (2001) Astrophysical constraints on a possible neutrino ball at the Galactic Center. A\&A376:853-860, astro-ph/0107497

Deccock L, Horsten L (2000) Quine,'Naturalized Epistemology, Perceptual Knowledge and Ontology', Lieven Deccock \& Leon Horsten (ediors), Editions Rodopi B.V., Amsterdam - Atlanta, GA 2000 ISBN 90-420-1241-2

Dékány I, Minniti D, Catelan M, Zoccali M, Saito RK, Hempel M, Gonzalez OA (2013) VVV Survey Near-infrared Photometry of Known Bulge RR Lyrae Stars: The Distance to the Galactic Center and Absence of a Barred Distribution of the Metal-poor Population. ApJL776:L19

DeWitt C, DeWitt BS (1973) Black holes. Lectures delivered at the Summer School of Theoretical Physics of the University of Grenoble at Les Houches; by DeWitt, C.; DeWitt, B. S.. New York, NY (USA): Gordon and Breach

Dexter J, Fragile PC (2013) Tilted black hole accretion disc models of Sagittarius A*: time-variable millimetre to near-infrared emission. MNRAS432:2252-2272

Dexter J, O'Leary RM (2014) The Peculiar Pulsar Population of the Central Parsec. ApJL783:L7

Dexter J, Agol E, Fragile PC, McKinney JC (2010) The Submillimeter Bump in Sgr A* from Relativistic MHD Simulations. ApJ717:1092-1104

Dexter J, Agol E, Fragile PC, McKinney JC (2012a) Radiative Models of Sagittarius A* and M87 from Relativistic MHD Simulations. Journal of Physics Conference Series 372(1):012023, 1202.0348

Dexter J, McKinney JC, Agol E (2012b) The size of the jet launching region in M87. MNRAS421:1517-1528, 1109.6011.

Do T, Ghez AM, Morris MR, Lu JR, Matthews K, Yelda S, Larkin J (2009) High Angular Resolution Integral-Field Spectroscopy of the Galaxy's Nuclear Cluster: A Missing Stellar Cusp? ApJ703:1323-1337, 0908.0311

Do T, Martinez GD, Yelda S, Ghez A, Bullock J, Kaplinghat M, Lu JR, Peter AHG, Phifer K (2013) Three-dimensional Stellar Kinematics at the Galactic Center: Measuring the Nuclear Star Cluster Spatial Density Profile, Black Hole Mass, and Distance. ApJL779:L6

Dobler G, Finkbeiner DP, Cholis I, Slatyer T, Weiner N (2010) The Fermi Haze: A Gamma-ray Counterpart to the Microwave Haze. ApJ717:825-842, 0910.4583

Doeleman SS, Lonsdale CJ, Greenhill LJ (1998) VLBI Imaging of the 86 GHz SiO Maser Emission in the Circumstellar Envelope of VX Sagittarii. ApJ494:400-408

Doeleman SS, Weintroub J, Rogers AEE, Plambeck R, Freund R, Tilanus RPJ, Friberg P, Ziurys LM, Moran JM, Corey B, Young KH, Smythe DL, Titus M, Marrone DP, Cappallo RJ, Bock DCJ, Bower GC, Chamberlin R, Davis GR, Krichbaum TP, Lamb J, Maness H, Niell AE, Roy A, Strittmatter P, Werthimer D, Whitney AR, Woody D (2008) Event-horizon-scale structure in the supermassive black hole candidate at the Galactic Centre. Nature455:78-80

Doeleman SS, Fish VL, Broderick AE, Loeb A, Rogers AEE (2009) Detecting Flaring Structures in Sagittarius A* with High-Frequency VLBI. ApJ695:59-74

Dvali G, Gomez C (2011) Black Hole's Quantum N-Portrait. arXiv11123359 and FortschPhys 61 (2013) 742-767 1112.3359

Dvali G, Gomez C (2013) Black hole's 1/N hair. arXiv:12036575; Physics Letters B 719:419-423, 1203.6575

Dvali G, Gomez C, Lüst D (2013) Black hole quantum mechanics in the presence of species. Fortschritte der Physik, Vol 61, Issue 7-8, pp 768-778; see also arXiv:12062365 61:768-778, 1206.2365

Dvali G, Gomez C, Lüst D (2016) Classical limit of black hole quantum N-portrait and BMS symmetry. Physics Letters B 753:173-177, 1509.02114

Earnshaw HM, Roberts TP, Heil LM, Mezcua M, Walton DJ, Done C, Harrison FA, Lansbury GB, Middleton MJ, Sutton AD (2016) A variable ULX and possible IMBH candidate in M51a. MNRAS456:3840-3854, 1512.04825

Eatough R, Lazio TJW, Casanellas J, Chatterjee S, Cordes JM, Demorest PB, Kramer M, Lee KJ, Liu K, Ransom SM, Wex N (2015) Observing Radio Pulsars in the Galactic Centre with the Square Kilometre Array. Advancing Astrophysics with the Square Kilometre Array (AASKA14) $45,1501.00281$

Eatough RP, Falcke H, Karuppusamy R, Lee KJ, Champion DJ, Keane EF, Desvignes G, Schnitzeler DHFM, Spitler LG, Kramer M, Klein B, Bassa C, Bower GC, Brunthaler A, Cognard I, Deller AT, Demorest PB, Freire PCC, Kraus A, Lyne AG, Noutsos A, Stappers B, Wex N (2013) A strong magnetic field around the supermassive black hole at the centre of the Galaxy. Nature501:391-394, arXiv1308.3147

Eckart A, Genzel R (1996) Observations of stellar proper motions near the Galactic Centre. Nature 383:415-417

Eckart A, Genzel R (1997) Stellar proper motions in the central 0.1 PC of the Galaxy. MNRAS284:576-598 
Eckart A, Genzel R, Ott T, Schödel R (2002) Stellar orbits near Sagittarius A*. MNRAS331:917934

Eckart A, Moultaka J, Viehmann T, Straubmeier C, Mouawad N, Genzel R, Ott T, Schödel R, Baganoff FK, Morris MR (2003) Monitoring Sagittarius A* in the MIR with the VLT. Astronomische Nachrichten Supplement 324:557-561

Eckart A, Schödel R, Straubmeier C (2005) The black hole at the center of the Milky Way / Andreas Eckart, Rainer Schödel, Christian Straubmeier. London:Imperial College Press ; Hackensack, NJ : Distributed by World Scientific Pub. Co., c2005.

Eckart A, Baganoff FK, Schödel R, Morris M, Genzel R, Bower GC, Marrone D, Moran JM, Viehmann T, Bautz MW, Brandt WN, Garmire GP, Ott T, Trippe S, Ricker GR, Straubmeier C, Roberts DA, Yusef-Zadeh F, Zhao JH, Rao R (2006a) The flare activity of Sagittarius A*. New coordinated mm to X-ray observations. A\&A450:535-555

Eckart A, Schödel R, Meyer L, Trippe S, Ott T, Genzel R (2006b) Polarimetry of near-infrared flares from Sagittarius A*. A\&A455:1-10

Eckart A, Schödel R, Straubmeier C, Bertram T, Pott JU, Muzic K, Meyer L, Moultaka J, Viehmann T, Rost S, Herbst T (2006c) Interferometric observations of the galactic center: LBT and VLTI. In: Society of Photo-Optical Instrumentation Engineers (SPIE) Conference Series, Society of Photo-Optical Instrumentation Engineers (SPIE) Conference Series, vol 6268, p 1

Eckart A, Zamaninasab M, Straubmeier C, Fischer S, Araujo-Hauck C, Garcia-Marin M, Wiest M, Witzel G, Buchholz RM, Sabha N, Muzic K, Eisenhauer F, Paumard T, Yazici S, Perrin G, Brandner W, Perraut K, Amorim A, Schöller M (2010) Signatures of strong gravity with GRAVITY. In: Society of Photo-Optical Instrumentation Engineers (SPIE) Conference Series, Society of Photo-Optical Instrumentation Engineers (SPIE) Conference Series, vol 7734, p 0

Eckart A, Britzen S, Horrobin M, Zamaninasab M, Muzic K, Sabha N, Shahzamanian B, Yazici S, Moser L, Zuther J, Garcia-Marin M, Valencia-S M, Bursa M, Karssen G, Karas V, Jalali B, Vitale M, Bremer M, Fischer S, Smajic S, Rauch C, Kunneriath D, Moultaka J, Straubmeier C, Rashed YE, Iserlohe C, Busch G, Markakis K, Borkar A, Zensus AJ (2012a) The Galactic Center as a pradigm for low-luminosity nuclei? What can be learned from SgrA* for the central engine and conditions of star formation in nuclei of Seyfert galaxies and low luminosity nearby QSOs; The K-band identification of the DSO/G2 source from VLT and Keck data. In: Proceedings of Nuclei of Seyfert galaxies and QSOs - Central engine and conditions of star formation (Seyfert 2012). 6-8 November, 2012. Max-Planck-Insitut fuer Radioastronomie (MPIfR), Bonn, Germany. arXiv1311.2743, p 4, 1311.2743

Eckart A, García-Marín M, Vogel SN, Teuben P, Morris MR, Baganoff F, Dexter J, Schödel R, Witzel G, Valencia-S M, Karas V, Kunneriath D, Straubmeier C, Moser L, Sabha N, Buchholz R, Zamaninasab M, Mužić K, Moultaka J, Zensus JA (2012b) Millimeter to X-ray flares from Sagittarius A*. A\&A537:A52

Eckart A, García-Marín M, Vogel SN, Teuben P, Morris MR, Baganoff F, Dexter J, Schödel R, Witzel G, Valencia-S M, Karas V, Kunneriath D, Straubmeier C, Moser L, Sabha N, Buchholz R, Zamaninasab M, Mužić K, Moultaka J, Zensus JA (2012c) Millimeter to X-ray flares from Sagittarius A*. A\&A537:A52

Eckart A, Sabha N, Witzel G, Straubmeier C, Shahzamanian B, Valencia-S M, García-Marín M, Horrobin M, Moser L, Zuther J, Fischer S, Rauch C, Rost S, Iserlohe C, Yazici S, Smajic S, Wiest M, Araujo-Hauck C, Wank I (2012d) Beating the confusion limit: the necessity of high angular resolution for probing the physics of Sagittarius A* and its environment: opportunities for LINCNIRVANA (LBT), GRAVITY (VLTI) and and METIS (E-ELT). In: Society of Photo-Optical Instrumentation Engineers (SPIE) Conference Series, Society of Photo-Optical Instrumentation Engineers (SPIE) Conference Series, vol 8445, p 1, 1208.1129

Einstein A (1936) Lens-Like Action of a Star by the Deviation of Light in the Gravitational Field. Science 84:506-507

Einstein A (1939) On a Stationary System with Spherical Symmetry Consisting of Many Gravitating Masses. Annals of Mathematics 40:922

Eisenhauer F, Schödel R, Genzel R, Ott T, Tecza M, Abuter R, Eckart A, Alexander T (2003) A Geometric Determination of the Distance to the Galactic Center. ApJL597:L121-L124

Eisenhauer F, Genzel R, Alexander T, Abuter R, Paumard T, Ott T, Gilbert A, Gillessen S, Horrobin M, Trippe S, Bonnet H, Dumas C, Hubin N, Kaufer A, Kissler-Patig M, Monnet G, Ströbele S, Szeifert T, Eckart A, Schödel R, Zucker S (2005) SINFONI in the Galactic Center: Young Stars and Infrared Flares in the Central Light-Month. ApJ628:246-259, astro-ph/0502129

Eisenhauer F, Perrin G, Brandner W, Straubmeier C, Richichi A, Gillessen S, Berger JP, Hippler S, Eckart A, Schöller M, Rabien S, Cassaing F, Lenzen R, Thiel M, Clénet Y, Ramos JR, Kellner S, Fédou P, Baumeister H, Hofmann R, Gendron E, Boehm A, Bartko H, Haubois X, Klein R, Dodds-Eden K, Houairi K, Hormuth F, Gräter A, Jocou L, Naranjo V, Genzel R, Kervella P, Henning T, Hamaus N, Lacour S, Neumann U, Haug M, Malbet F, Laun W, Kolmeder J, Paumard T, Rohloff RR, Pfuhl O, Perraut K, Ziegleder J, Rouan D, Rousset G (2008) GRAVITY: getting to the event horizon of Sgr A*. In: Society of Photo-Optical 
Instrumentation Engineers (SPIE) Conference Series, Society of Photo-Optical Instrumentation Engineers (SPIE) Conference Series, vol 7013, p 2, 0808.0063

Ellis HG (1973) Ether flow through a drainhole: A particle model in general relativity. Journal of Mathematical Physics 14:104-118

Emmanoulopoulos D (2015) Pinpointing the base of the AGN jets through general relativistic X-ray reverberation studies. In: Massaro F, Cheung CC, Lopez E, Siemiginowska A (eds) IAU Symposium, IAU Symposium, vol 313, pp 346-351, 1411.0727

Esteban EP, Ramos E (1988) Rotating black hole in an external electromagnetic field. Phys. Rev. D38:2963-2971

Ewing E Ann (1964) 'Black Holes' in Space. In: for Science S, the Public (eds) Science News Letters, January 18, 1964, p 39

Falcke H, Markoff S (2000) The jet model for Sgr A*: Radio and X-ray spectrum. A\&A362:113-118

Falcke H, Melia F, Agol E (2000) Viewing the Shadow of the Black Hole at the Galactic Center. ApJL528:L13-L16

Falcke H, Markoff S, Bower GC (2009) Jet-lag in Sagittarius A*: what size and timing measurements tell us about the central black hole in the Milky Way. A\&A496:77-83, 0901.3723

Ferrarese L, Côté P, Dalla Bontà E, Peng EW, Merritt D, Jordán A, Blakeslee JP, Haşegan M, Mei S, Piatek S, Tonry JL, West MJ (2006) A Fundamental Relation between Compact Stellar Nuclei, Supermassive Black Holes, and Their Host Galaxies. ApJL644:L21-L24, astro-ph/0603840

Ferré-Mateu A, Mezcua M, Trujillo I, Balcells M, van den Bosch RCE (2015) Massive Relic Galaxies Challenge the Co-evolution of Super-massive Black Holes and Their Host Galaxies. ApJ808:79, 1506.02663

Finkelstein D (1958) Past-Future Asymmetry of the Gravitational Field of a Point Particle. Phys Rev 110, 965110

Fish VL, Doeleman SS, Beaudoin C, Blundell R, Bolin DE, Bower GC, Chamberlin R, Freund R, Friberg P, Gurwell MA, Honma M, Inoue M, Krichbaum TP, Lamb J, Marrone DP, Moran JM, Oyama T, Plambeck R, Primiani R, Rogers AEE, Smythe DL, SooHoo J, Strittmatter P, Tilanus RPJ, Titus M, Weintroub J, Wright M, Woody D, Young KH, Ziurys LM (2011) 1.3 mm Wavelength VLBI of Sagittarius A*: Detection of Time-variable Emission on Event Horizon Scales. ApJL727:L36

Fish VL, Johnson MD, Lu RS, Doeleman SS, Bouman KL, Zoran D, Freeman WT, Psaltis D, Narayan R, Pankratius V, Broderick AE, Gwinn CR, Vertatschitsch LE (2014) Imaging an Event Horizon: Mitigation of Scattering toward Sagittarius A*. ApJ795:134, 1409.4690

Fish VL, Johnson MD, Doeleman SS, Broderick AE, Psaltis D, Lu RS, Akiyama K, Alef W, Algaba JC, Asada K, Beaudoin C, Bertarini A, Blackburn L, Blundell R, Bower GC, Brinkerink C, Cappallo R, Chael AA, Chamberlin R, Chan CK, Crew GB, Dexter J, Dexter M, Dzib SA, Falcke H, Freund R, Friberg P, Greer CH, Gurwell MA, Ho PTP, Honma M, Inoue M, Johannsen T, Kim J, Krichbaum TP, Lamb J, León-Tavares J, Loeb A, Loinard L, MacMahon D, Marrone DP, Moran JM, Mościbrodzka M, Ortiz-León GN, Oyama T, Özel F, Plambeck RL, Pradel N, Primiani RA, Rogers AEE, Rosenfeld K, Rottmann H, Roy AL, Ruszczyk C, Smythe DL, SooHoo J, Spilker J, Stone J, Strittmatter P, Tilanus RPJ, Titus M, Vertatschitsch L, Wagner J, Wardle JFC, Weintroub J, Woody D, Wright M, Yamaguchi P, Young A, Young KH, Zensus JA, Ziurys LM (2016) Persistent Asymmetric Structure of Sagittarius A* on Event Horizon Scales. ApJ820:90

Francis C, Anderson E (2014) Two estimates of the distance to the Galactic Centre. MNRAS441:1105-1114

Franklin A (2016) What Makes a Good Experiment? University of Pittsburgh Press ISBN-13 9780822944416

Freitag M (2003) Gravitational waves from stars orbiting the sagittarius a* black hole. The Astrophysical Journal Letters 583(1):L21

Fritz TK, Gillessen S, Dodds-Eden K, Martins F, Bartko H, Genzel R, Paumard T, Ott T, Pfuhl O, Trippe S, Eisenhauer F, Gratadour D (2010) GC-IRS13E - A Puzzling Association of Three Early-type Stars. ApJ721:395-411, 1003.1717

Gair JR, Barack L, Creighton T, Cutler C, Larson SL, Phinney ES, Vallisneri M (2004) Event rate estimates for LISA extreme mass ratio capture sources. Classical and Quantum Gravity 21:S1595-S1606

Gair JR, Vallisneri M, Larson SL, Baker JG (2013) Testing General Relativity with Low-Frequency, Space-Based Gravitational-Wave Detectors. Living Reviews in Relativity 16:7

Galison P (1987) How Experiments End; The University of Chicago Press Books; ISBN: 9780226279152 Published October 1987

García Díaz A (1985) Magnetic generalization of the Kerr-Newman metric. Journal of Mathematical Physics 26:155-156

Genzel R, Pichon C, Eckart A, Gerhard OE, Ott T (2000) Stellar dynamics in the Galactic Centre: proper motions and anisotropy. MNRAS317:348-374, astro-ph/0001428

Genzel R, Schödel R, Ott T, Eckart A, Alexander T, Lacombe F, Rouan D, Aschenbach B (2003) Near-infrared flares from accreting gas around the supermassive black hole at the Galactic Centre. Nature425:934-937, astro-ph/0310821 
Genzel R, Eisenhauer F, Gillessen S (2010) The Galactic Center massive black hole and nuclear star cluster. Reviews of Modern Physics 82:3121-3195, 1006.0064

Ghez A, Morris M, Lu J, Weinberg N, Matthews K, Alexander T, Armitage P, Becklin E, Brown W, Campbell R, Do T, Eckart A, Genzel R, Gould A, Hansen B, Ho L, Lo F, Loeb A, Melia F, Merritt D, Milosavljevic M, Perets H, Rasio F, Reid M, Salim S, Schödel R, Yelda S (2009) The Galactic Center: A Laboratory for Fundamental Astrophysics and Galactic Nuclei. In: astro2010: The Astronomy and Astrophysics Decadal Survey, Astronomy, vol 2010, 0903.0383

Ghez AM, Klein BL, Morris M, Becklin EE (1998) High Proper-Motion Stars in the Vicinity of Sagittarius A*: Evidence for a Supermassive Black Hole at the Center of Our Galaxy. ApJ509:678-686, astro-ph/9807210

Ghez AM, Duchene G, Morris M, Becklin EE, Hornstein SD, Tanner A, Kremenek T, Matthews K, Thompson D, Soifer BT, Larkin J, McLean I (2002) Full 3-D Orbital Solutions for Stars Making a Close Approach to the Supermassive Black Hole at the Center of the Galaxy. In: American Astronomical Society Meeting Abstracts, Bulletin of the American Astronomical Society, vol 34, p 1219

Ghez AM, Wright SA, Matthews K, Thompson D, Le Mignant D, Tanner A, Hornstein SD, Morris M, Becklin EE, Soifer BT (2004) Variable Infrared Emission from the Supermassive Black Hole at the Center of the Milky Way. ApJL601:L159-L162, astro-ph/0309076

Ghez AM, Salim S, Weinberg NN, Lu JR, Do T, Dunn JK, Matthews K, Morris MR, Yelda S, Becklin EE, Kremenek T, Milosavljevic M, Naiman J (2008) Measuring Distance and Properties of the Milky Way's Central Supermassive Black Hole with Stellar Orbits. ApJ689:1044-1062

Ghisellini G, Foschini L, Volonteri M, Ghirlanda G, Haardt F, Burlon D, Tavecchio F (2009) The blazar S5 0014+813: a real or apparent monster? MNRAS399:L24-L28, 0906.0575

Ghisellini G, Della Ceca R, Volonteri M, Ghirlanda G, Tavecchio F, Foschini L, Tagliaferri G, Haardt F, Pareschi G, Grindlay J (2010) Chasing the heaviest black holes of jetted active galactic nuclei. MNRAS405:387-400, 0912.0001

Giannios D, Lorimer DR (2016) Flares from Galactic Centre pulsars: a new class of X-ray transients? MNRAS459:L95-L99

Giddings SB (2014) Possible observational windows for quantum effects from black holes. Phys. Rev. D90(12):124033, 1406.7001

Gillessen S, Eisenhauer F, Quataert E, Genzel R, Paumard T, Trippe S, Ott T, Abuter R, Eckart A, Lagage PO, Lehnert MD, Tacconi LJ, Martins F (2006) Variations in the Spectral Slope of Sagittarius A* during a Near-Infrared Flare. ApJL640:L163-L166, astro-ph/0511302

Gillessen S, Eisenhauer F, Fritz TK, Bartko H, Dodds-Eden K, Pfuhl O, Ott T, Genzel R (2009a) The Orbit of the Star S2 Around SGR A* from Very Large Telescope and Keck Data. ApJL707:L114-L117

Gillessen S, Eisenhauer F, Trippe S, Alexander T, Genzel R, Martins F, Ott T (2009b) Monitoring Stellar Orbits Around the Massive Black Hole in the Galactic Center. ApJ692:1075-1109

Gillessen S, Plewa PM, Eisenhauer F, Sari R, Waisberg I, Habibi M, Pfuhl O, George E, Dexter J, von Fellenberg S, Ott T, Genzel R (2017) An Update on Monitoring Stellar Orbits in the Galactic Center. ApJ837:30, DOI 10.3847/1538-4357/aa5c41, 1611.09144

Graham AW, Scott N, Schombert JM (2015) Super-Massive Black Hole Mass Scaling Relations. Publication of Korean Astronomical Society 30:335-339, 1411.1438

Grenzebach A, Perlick V, Lämmerzahl C (2015) Photon regions and shadows of accelerated black holes. International Journal of Modern Physics D 24:1542024

Guedes J, Madau P, Mayer L, Callegari S (2011) Recoiling Massive Black Holes in Gas-rich Galaxy Mergers. ApJ729:125, 1008.2032

Gwinn CR, Kovalev YY, Johnson MD, Soglasnov VA (2014) Discovery of Substructure in the Scatter-broadened Image of Sgr A*. ApJL794:L14, 1409.0530

Hacking I (ed) (1983) Representing and Intervening, page 24. Cambridge University Press

Hacking I (1989) Extragalactic reality: The case of gravitational lensing. Philosophy of Science $56: 555-581$

Haggard HM, Rovelli C (2015) Quantum-gravity effects outside the horizon spark black to white hole tunneling. Phys. Rev. D92(10):104020

Hawking S (2014) Information Preservation and Waether Forcasting for Black Holes. arXiv: 14015761

Hawking S, Penrose R (1996) The Nature of Space and Time. Princeton University Press, Princeton

Hawking SW, Israel W (1987) Three hundred years of gravitation, Cambridge University Press

Herdeiro CAR, Radu E (2014) Kerr Black Holes with Scalar Hair. Physical Review Letters $112(22): 221101,1403.2757$

Heusler M (1996) Black hole uniqueness theorems. Cambridge University Press, Cambridge

Hoefer C, Smeenk C (2016) Philosophy of the physical sciences. In Oxford Handbook of the Philosophy of Science, ed by Paul Humphreys Oxford: Oxford University Press (published online 2015 ; in print Sept 2016

Hopman C, Alexander T (2006) Resonant Relaxation near a Massive Black Hole: The Stellar Distribution and Gravitational Wave Sources. ApJ645:1152-1163 
Horrobin M, Eisenhauer F, Tecza M, Thatte N, Genzel R, Abuter R, Iserlohe C, Schreiber J, Schegerer A, Lutz D, Ott T, Schödel R (2004) First results from SPIFFI. I: The Galactic Center. Astronomische Nachrichten 325:88-91

Hughes SA (2000) Evolution of circular, nonequatorial orbits of Kerr black holes due to gravitational-wave emission. Physical Review D 61(8):084004

Hughes SA (2001) Evolution of circular, nonequatorial orbits of Kerr black holes due to gravitational-wave emission. II. Inspiral trajectories and gravitational waveforms. Physical Review D 64(6):064004

Israel W (1987) Dark stars: the evolution of an idea. In: Three Hundred Years of Gravitation; editor Hawking, S. W. and Israel, W., pp 199-276

Iwata K, Yamazaki S, Mutombo P, Hapala P, Ondráček M, Jelínek P, Sugimoto Y (2015) Chemical structure imaging of a single molecule by atomic force microscopy at room temperature. Nature Communications 6:7766

Jaroszynski M (1999) Prospects for the Determination of Star Orbits near the Galactic Center. ApJ521:591-596, astro-ph/9903354

Johannsen T (2015) Sgr A* and General Relativity. ArXiv 151203818 [astro-phGA] 1512.03818

Johannsen T (2016) Testing the no-hair theorem with observations of black holes in the electromagnetic spectrum. Classical and Quantum Gravity 33(12):124001, 1602.07694

Johnson MD, Fish VL, Doeleman SS, Marrone DP, Plambeck RL, Wardle JFC, Akiyama K, Asada K, Beaudoin C, Blackburn L, Blundell R, Bower GC, Brinkerink C, Broderick AE, Cappallo R, Chael AA, Crew GB, Dexter J, Dexter M, Freund R, Friberg P, Gold R, Gurwell MA, Ho PTP, Honma M, Inoue M, Kosowsky M, Krichbaum TP, Lamb J, Loeb A, Lu RS, MacMahon D, McKinney JC, Moran JM, Narayan R, Primiani RA, Psaltis D, Rogers AEE, Rosenfeld K, SooHoo J, Tilanus RPJ, Titus M, Vertatschitsch L, Weintroub J, Wright M, Young KH, Zensus JA, Ziurys LM (2015) Resolved magnetic-field structure and variability near the event horizon of Sagittarius A*. Science 350:1242-1245, 1512.01220

Karas V, Vokrouhlický D (1991) On interpretation of the magnetized Kerr-Newman black hole. Journal of Mathematical Physics 32:714-716

Karas V, Huré JM, Semerák O (2004) TOPICAL REVIEW: Gravitating discs around black holes. Classical and Quantum Gravity 21:1, astro-ph/0401345

Karas V, Kopáček O, Kunneriath D (2012) Influence of frame-dragging on magnetic null points near rotating black holes. Classical and Quantum Gravity 29(3):035010, 1201.0009

Kardashev NS, Andreyanov VV, Gromov VD, Buyakas VI, Gvamichava AS, Kotik AN, Kurt VG, Lazareva GS, Mironova EN, Myshonkova NV, Slysh VI, Trubnikov AG, Vinogradov IS, Troitsky VF, Puryaev DT, Usyukin VI (2007) The Millimetron Project. In: Kardashev NS, Dagkesamanskii SA (eds) Radioastronomical Tools and Techniques, p 111

Kardashev NS, Novikov ID, Lukash VN, Pilipenko SV, Mikheeva EV, Bisikalo DV, Wiebe DS, Doroshkevich AG, Zasov AV, Zinchenko II, Ivanov PB, Kostenko VI, Larchenkova TI, Likhachev SF, Malov IF, Malofeev VM, Pozanenko AS, Smirnov AV, Sobolev AM, Cherepashchuk AM, Shchekinov YA (2014) Review of scientific topics for the Millimetron space observatory. Physics Uspekhi 57:1199-1228

Kaup DJ (1968) Klein-Gordon Geon. Physical Review 172:1331-1342

Kerr RP (1963) Gravitational Field of a Spinning Mass as an Example of Algebraically Special Metrics. Physical Review Letters 11:237-238

Kiefer C (1999) Thermodynamics of black holes and Hawking radiation. "Classical and Quantum Black Holes", ed by P Fré, V Gorini, G Magli, \& U Moschella, Institute of Physics Publishing, Bristol and Philadelphia, pp 17-74

Kiefer C (2012) Quantum Gravity. Third edition, Oxford University Press, Oxford

Kiefer C (2015) Quantum black hole without singularity. arXiv:151208346, invited contribution to the BH6 session at the Marcel Grossmann Conference MG14 on General Relativity and Quantum Cosmology, WSPC Proceedings

Kiefer C, Marto J, Moniz PV (2009) Indefinite oscillators and black hole evaporation. Ann Phys (Berlin), 18, 722

King A (2016) How big can a black hole grow? MNRAS456:L109-L112

Kiziltan B, Baumgardt H, Loeb A (2017) An intermediate-mass black hole in the centre of the globular cluster 47 Tucanae. Nature542:203-205, 1702.02149

Kormendy J, Ho LC (2013) Coevolution (Or Not) of Supermassive Black Holes and Host Galaxies. ARA\&A51:511-653

Krabbe A, Genzel R, Eckart A, Najarro F, Lutz D, Cameron M, Kroker H, Tacconi-Garman LE, Thatte N, Weitzel L, Drapatz S, Geballe T, Sternberg A, Kudritzki R (1995) The Nuclear Cluster of the Milky Way: Star Formation and Velocity Dispersion in the Central 0.5 Parsec. ApJL447:L95

Laudan L, Leplin J (1991) Empirical equivalence and underdetermination larry laudan \& jarrett leplin journal of philosophy 88 (9):449-472 (1991). Journal of Philosophy 88:449-472

Leinert C, Graser U (1998) MIDI: a mid-infrared interferometric instrument for the VLTI. In: Reasenberg RD (ed) Astronomical Interferometry, Society of Photo-Optical Instrumentation Engineers (SPIE) Conference Series, vol 3350, pp 389-393 
Li Z, Morris MR, Baganoff FK (2013) Evidence for a Parsec-scale Jet from the Galactic Center Black Hole: Interaction with Local Gas. ApJ779:154

Löckmann U, Baumgardt H (2008) Tracing intermediate-mass black holes in the Galactic Centre. MNRAS384:323-330, 0711.1326

Lu RS, Roelofs F, Fish VL, Shiokawa H, Doeleman SS, Gammie CF, Falcke H, Krichbaum TP, Zensus JA (2016) Imaging an Event Horizon: Mitigation of Source Variability of Sagittarius A*. ApJ817:173, 1512.08543

Lu Y, Torres DF (2003) The Relativistic Iron K $\alpha$ Line from an Accretion Disc Onto a Static Non-Baryonic Compact Object. International Journal of Modern Physics D 12:63-77, astro-ph/0205418

Luminet JP (1979) Image of a spherical black hole with thin accretion disk. A\&A75:228-235

Maillard JP, Paumard T, Stolovy SR, Rigaut F (2004) The nature of the Galactic Center source IRS 13 revealed by high spatial resolution in the infrared. A\&A423:155-167, astro-ph/0404450

Majaess DJ, Turner DG, Lane DJ (2009) Characteristics of the Galaxy according to Cepheids. MNRAS398:263-270

Malkin ZM (2013) Analysis of determinations of the distance between the sun and the galactic center. Astronomy Reports 57:128-133

Maoz E (1998) Dynamical Constraints on Alternatives to Supermassive Black Holes in Galactic Nuclei. ApJL494:L181-L184, astro-ph/9710309

Marcus R (2015) The Eleatic and the Indispensabilist. THEORIA 30/3, 415-429

Markakis K, Dierkes J, Eckart A, Nishiyama S, Britzen S, García-Marín M, Horrobin M, Muxlow T, Zensus JA (2015) Subaru and e-Merlin observations of NGC 3718. Diaries of a supermassive black hole recoil? A\&A580:A11, 1504.03691

Markoff S (2005) Sagittarius A* in Context: Daily Flares as a Probe of the Fundamental X-Ray Emission Process in Accreting Black Holes. ApJL618:L103-L106

Markoff S, Bower GC, Falcke H (2007) How to hide large-scale outflows: size constraints on the jets of Sgr A*. MNRAS379:1519-1532

Markoff S, Nowak MA, Maitra D, Wilms J, Gallo E, Hynes R, Plotkin R (2011) Fitting along the Fundamental Plane: New comparisons of jet physics across the black hole mass scale. In: Romero GE, Sunyaev RA, Belloni T (eds) Jets at All Scales, IAU Symposium, vol 275, pp $250-254$

Matsuoka Y, Strauss MA, Shen Y, Brandt WN, Greene JE, Ho LC, Schneider DP, Sun M, Trump JR (2015) The Sloan Digital Sky Survey Reverberation Mapping Project: Post-Starburst Signatures in Quasar Host Galaxies at $\mathrm{z}<1$. ApJ811:91, 1506.07535

Mazur PO, Mottola E (2001) Gravitational Condensate Stars: An Alternative to Black Holes. ArXiv General Relativity and Quantum Cosmology e-prints, gr-qc/0109035 gr-qc/0109035

Melia F, Falcke H (2001) The Supermassive Black Hole at the Galactic Center. ARA\&A39:309-352, astro-ph/0106162

Menten KM, Reid MJ, Eckart A, Genzel R (1997) The Position of Sagittarius A*: Accurate Alignment of the Radio and Infrared Reference Frames at the Galactic Center. ApJL475:L111L114

Merritt D (2010) The Distribution of Stars and Stellar Remnants at the Galactic Center. ApJ718:739-761, 0909.1318

Merritt D, Alexander T, Mikkola S, Will CM (2010) Testing properties of the Galactic center black hole using stellar orbits. Phys. Rev. D81(6):062002

Meyer L, Schödel R, Eckart A, Karas V, Dovčiak M, Duschl WJ (2006) K-band polarimetry of an Sgr A* flare with a clear sub-flare structure. A\&A458:L25-L28

Meyer L, Schödel R, Eckart A, Duschl WJ, Karas V, Dovčiak M (2007) On the orientation of the Sagittarius A* system. A\&A473:707-710

Mielke EW, Schunck FE (2000) Boson stars: alternatives to primordial black holes? Nuclear Physics B 564:185-203, gr-qc/0001061

Mielke EW, Schunck FE (2002) Boson and Axion Stars. In: Gurzadyan VG, Jantzen RT, Ruffini R (eds) The Ninth Marcel Grossmann Meeting, pp 581-591

Miller JM, Tomsick JA, Bachetti M, Wilkins D, Boggs SE, Christensen FE, Craig WW, Fabian AC, Grefenstette BW, Hailey CJ, Harrison FA, Kara E, King AL, Stern DK, Zhang WW (2015) New Constraints on the Black Hole Low/Hard State Inner Accretion Flow with NuSTAR. ApJL799:L6, 1411.1921

Miller MC (2006) Constraints on alternatives to supermassive black holes. MNRAS367:L32-L36, astro-ph/0512194

Misner C, Thorne K, Wheeler J (1973) Gravitation, San Francisco: W. H. Freeman, ISBN 978-07167-0344-0

Montero B (2003) Varieties of causal closure; Chapter 8 in Physicalism and Mental Causation: The Metaphysics of Mind and Action; edited by Sven Walter; Heinz-Dieter Heckmann; Imprint Academic. pp. 173 ff. ISBN 0907845460.

Mori K, Gotthelf EV, Zhang S, An H, Baganoff FK, Barrière NM, Beloborodov AM, Boggs SE, Christensen FE, Craig WW, Dufour F, Grefenstette BW, Hailey CJ, Harrison FA, Hong J, Kaspi VM, Kennea JA, Madsen KK, Markwardt CB, Nynka M, Stern D, Tomsick JA, Zhang WW 
(2013) NuSTAR Discovery of a 3.76 s Transient Magnetar Near Sagittarius A*. ApJL770:L23, 1305.1945

Morris M, Howard C, Muno M, Baganoff FK, Park S, Feigelson E, Garmire G, Brandt N (2004) The Hot Interstellar Medium of the Galactic Center: Observations with Chandra. In: Pfalzner S, Kramer C, Staubmeier C, Heithausen A (eds) The Dense Interstellar Medium in Galaxies, p 281

Mościbrodzka M, Falcke H (2013) Coupled jet-disk model for Sagittarius A*: explaining the flatspectrum radio core with GRMHD simulations of jets. A\&A559:L3

Mościbrodzka M, Falcke H, Shiokawa H, Gammie CF (2014) Observational appearance of inefficient accretion flows and jets in 3D GRMHD simulations: Application to Sagittarius A*. A\&A570:A7

Mossoux E, Grosso N, Vincent FH, Porquet D (2015) Study of the X-ray activity of Sagittarius A* during the 2011 XMM-Newton campaign. A\&A573:A46, 1409.6452

Mou G, Yuan F, Bu D, Sun M, Su M (2014) Fermi Bubbles Inflated by Winds Launched from the Hot Accretion Flow in Sgr A*. ApJ790:109, 1403.2129

Mou G, Yuan F, Gan Z, Sun M (2015) The Accretion Wind Model of Fermi Bubbles. II. Radiation. ApJ811:37, 1505.00892

Mužić K, Eckart A, Schödel R, Meyer L, Zensus A (2007) First proper motions of thin dust filaments at the Galactic center. A\&A469:993-1002

Mužić K, Eckart A, Schödel R, Buchholz R, Zamaninasab M, Witzel G (2010) Comet-shaped sources at the Galactic center. Evidence of a wind from the central 0.2 pc. A\&A521:A13

Nakamura M, Asada K (2013) The Parabolic Jet Structure in M87 as a Magnetohydrodynamic Nozzle. ApJ775:118, 1308.1436

Narayan R, Yi I, Mahadevan R (1995) Explaining the spectrum of Sagittarius A* with a model of an accreting black hole. Nature374:623-625

Narayan R, Mahadevan R, Grindlay JE, Popham RG, Gammie C (1998) Advection-dominated accretion model of Sagittarius A*: evidence for a black hole at the Galactic center. ApJ492:554568

Natarajan P, Treister E (2009) Is there an upper limit to black hole masses? MNRAS393:838-845, 0808.2813

Nayakshin S, Power C, King AR (2012) The Observed M- $\sigma$ Relations Imply That Super-massive Black Holes Grow by Cold Chaotic Accretion. ApJ753:15, 1203.3450

Neilsen J, Markoff S, Nowak MA, Dexter J, Witzel G, Barrière N, Li Y, Baganoff FK, Degenaar N, Fragile PC, Gammie C, Goldwurm A, Grosso N, Haggard D (2015a) The X-Ray Flux Distribution of Sagittarius A* as Seen by Chandra. ApJ799:199

Neilsen J, Markoff S, Nowak MA, Dexter J, Witzel G, Barrière N, Li Y, Baganoff FK, Degenaar N, Fragile PC, Gammie C, Goldwurm A, Grosso N, Haggard D (2015b) The X-Ray Flux Distribution of Sagittarius A* as Seen by Chandra. ApJ799:199, 1412.3106

Nelson O John (2010) Hume's 'New Scene of Thought' and The Several Faces of David Hume in the Dialogues Concerning Natural Religion; with a preface by Jeff Broome; University Press of America, 2010, ISBN 978-0-7618-4735-9

Neugebauer G, Meinel R (1993) The Einsteinian gravitational field of the rigidly rotating disk of dust. ApJL414:L97-L99

Newmann A (2002) The Correspondence Theory of Truth; An Essay on the Metaphysics of Predication; Part of Cambridge Studies in Philosophy; Author: Andrew Newman, University of Nebraska, Omaha; Date Published: July 2002; ISBN: 9780521811392

Nordström G (1918) On the Energy of the Gravitation field in Einstein's Theory. Koninklijke Nederlandse Akademie van Wetenschappen Proceedings Series B Physical Sciences 20:12381245

Oka T, Mizuno R, Miura K, Takekawa S (2016) Signature of an Intermediate-mass Black Hole in the Central Molecular Zone of Our Galaxy. ApJL816:L7

Oppenheimer JR, Snyder H (1939) On Continued Gravitational Contraction. Physical Review 56:455-459

Oppenheimer JR, Volkoff GM (1939) On Massive Neutron Cores. Physical Review 55:374-381

Ostriker J (1964) The Equilibrium of Self-Gravitating Rings. ApJ140:1067

de Oteyza D, Gorman P, et al (2013) Direct Imaging of Covalent Bond Structure in Single-Molecule Chemical Reactions. Science 340:1434

Parsa M, Eckart A, et al (2017) Investigating relativistic motion of stars near the supermassive black hole in the Galactic center. A\&A, in preparation

Pasham DR, Strohmayer TE, Mushotzky RF (2014) A 400-solar-mass black hole in the galaxy M82. Nature513:74-76

Paumard T, Perrin G, Eckart A, Genzel R, Lena P, Schoedel R, Eisenhauer F, Mueller T, Gillessen S (2005) Scientific prospects for VLTI in the Galactic Centre: Getting to the Schwarzschild radius. Astronomische Nachrichten 326:568-568

Paumard T, Perrin G, Eckart A, Genzel R, Léna P, Schödel R, Eisenhauer F, Müller T, Gillessen S (2008) Scientific Prospects for VLTI in the Galactic Centre: Getting to the Schwarzschild Radius. In: Richichi A, Delplancke F, Paresce F, Chelli A (eds) The Power of Optical/IR Interferometry: Recent Scientific Results and 2nd Generation, p 313 
Peters PC, Mathews J (1963) Gravitational Radiation from Point Masses in a Keplerian Orbit. Physical Review 131:435-440

Pierro V, Pinto IM, Spallicci AD, Laserra E, Recano F (2001) Fast and accurate computational tools for gravitational waveforms from binary stars with any orbital eccentricity. MNRAS325:358-372, gr-qc/0005044

Plewa PM, Gillessen S, Eisenhauer F, Ott T, Pfuhl O, George E, Dexter J, Habibi M, Genzel R, Reid MJ, Menten KM (2015) Pinpointing the near-infrared location of Sgr A* by correcting optical distortion in the NACO imager. MNRAS453:3234-3244, DOI 10.1093/mnras/stv1910, 1509.01941

Poisson E (2004) A Relativist's Toolkit - The Mathematics of Black-Hole Mechanics. A Relativist's Toolkit - The Mathematics of Black-Hole Mechanics, Cambridge University Press, Cambridge

Pollney D, Reisswig C (2011) Gravitational Memory in Binary Black Hole Mergers. ApJL732:L13, 1004.4209

Porquet D, Grosso N, Predehl P, Hasinger G, Yusef-Zadeh F, Aschenbach B, Trap G, Melia F, Warwick RS, Goldwurm A, Bélanger G, Tanaka Y, Genzel R, Dodds-Eden K, Sakano M, Ferrando P (2008) X-ray hiccups from Sagittarius A* observed by XMM-Newton. The second brightest flare and three moderate flares caught in half a day. A\&A488:549-557, 0806.4088

Pott JU, Eckart A, Glindemann A, Viehmann T, Schodel R, Straubmeier C, Leinert C, Feldt M, Genzel R, Robberto M (2005) VLTI Observations of IRS 3: The brightest compact MIR source at the Galactic Centre. The Messenger 119:43-44, astro-ph/0505189

Pott JU, Eckart A, Glindemann A, Schödel R, Viehmann T, Robberto M (2008) The enigma of GCIRS 3. Constraining the properties of the mid-infrared reference star of the central parsec of the Milky Way with optical long-baseline interferometry. A\&A480:115-131, 0711.0249

Price H (2007) Quining Naturalism. Journal of Philosophy, 104, p375-402

Psaltis D (2012) The Influence of Gas Dynamics on Measuring the Properties of the Black Hole in the Center of the Milky Way with Stellar Orbits and Pulsars. ApJ759:130, 1112.0026

Psaltis D, Narayan R, Fish VL, Broderick AE, Loeb A, Doeleman SS (2015) Event Horizon Telescope Evidence for Alignment of the Black Hole in the Center of the Milky Way with the Inner Stellar Disk. ApJ798:15

Psaltis D, Wex N, Kramer M (2016a) A Quantitative Test of the No-hair Theorem with Sgr A* Using Stars, Pulsars, and the Event Horizon Telescope. ApJ818:121, 1510.00394

Psaltis D, Wex N, Kramer M (2016b) A Quantitative Test of the No-hair Theorem with Sgr A* Using Stars, Pulsars, and the Event Horizon Telescope. ApJ818:121, 1510.00394

Psillos S (2011a) Living with the abstract: realism and models. Synthese 180:3-17

Psillos S (2011b) Making Contact with Molecules: On Perrin and Achinstein. in Gregory J Morgan (ed), Philosophy of Science Matters: The Philosophy of Peter Achinstein, Oxford University Press 177- 190

Quataert E (2003) Radiatively Inefficient Accretion Flow Models of Sgr A*. Astronomische Nachrichten Supplement 324:435-443, astro-ph/0304099

Quine W (1948) On What There Is. Review of Metaphysics, 2 (1948/1949) p21-38

Rauch C, Ros E, Krichbaum TP, Eckart A, Zensus JA, Shahzamanian B, Mužić K (2016) Wisps in the Galactic center: Near-infrared triggered observations of the radio source Sgr A* at 43 GHz. A\&A587:A37, 1512.07509

Rees MJ (1984) Black Hole Models for Active Galactic Nuclei. ARA\&A22:471-506

Reid MJ (1993) The distance to the center of the Galaxy. ARA\&A31:345-372

Reid MJ, Menten KM, Genzel R, Ott T, Schödel R, Brunthaler A (2003a) The Position, Motion, and Mass of Sgr A*. Astronomische Nachrichten Supplement 324:505-511

Reid MJ, Menten KM, Genzel R, Ott T, Schödel R, Eckart A (2003b) The Position of Sagittarius A*. II. Accurate Positions and Proper Motions of Stellar SiO Masers near the Galactic Center. ApJ587:208-220, astro-ph/0212273

Reissner H (1916) Über die Eigengravitation des elektrischen Feldes nach der Einsteinschen Theorie. Annalen der Physik 355:106-120

Renn J, Sauer T (2005) In the limelight of stars. Einstein, Mandl, and the origins of gravitational lens research (German Title: Im Rampenlicht der Sterne. Einstein, Mandl und die Ursprünge der Gravitationslinsenforschung). Acta Historica Astronomiae 27:210-239

Revnivtsev MG, Churazov EM, Sazonov SY, Sunyaev RA, Lutovinov AA, Gilfanov MR, Vikhlinin AA, Shtykovsky PE, Pavlinsky MN (2004) Hard X-ray view of the past activity of Sgr A* in a natural Compton mirror. A\&A425:L49-L52, astro-ph/0408190

Reynolds CS (2014) Measuring Black Hole Spin Using X-Ray Reflection Spectroscopy. Space Sci. Rev.183:277-294, 1302.3260

Ricarte A, Dexter J (2015) The Event Horizon Telescope: exploring strong gravity and accretion physics. MNRAS446:1973-1987

Roedig C, Sesana A (2012) Origin and Implications of high eccentricities in massive black hole binaries at sub-pc scales. Journal of Physics Conference Series 363(1):012035, 1111.3742

Rovelli C, Vidotto F (2014) Planck stars. International Journal of Modern Physics D 23:1442026

Rozanska A, Kunneriath D, Czerny B, Adhikari TP, Karas V (2017) Multiphase environment of compact galactic nuclei: the role of the nuclear star cluster. MNRAS464:2090-2102 
Rubilar GF, Eckart A (2001) Periastron shifts of stellar orbits near the Galactic Center. A\&A374:95-104

Ruffini R, Bonazzola S (1969) Systems of Self-Gravitating Particles in General Relativity and the Concept of an Equation of State. Physical Review 187:1767-1783

Sabha N, Eckart A, Merritt D, Zamaninasab M, Witzel G, García-Marín M, Jalali B, Valencia-S M, Yazici S, Buchholz R, Shahzamanian B, Rauch C, Horrobin M, Straubmeier C (2012) The S-star cluster at the center of the Milky Way. On the nature of diffuse NIR emission in the inner tenth of a parsec. A\&A545:A70, 1203.2625

Sakai N, Saida H, Tamaki T (2014) Gravastar shadows. Phys. Rev. D90(10):104013, 1408.6929

Salpeter EE (1964) Accretion of Interstellar Matter by Massive Objects. ApJ140:796-800

Sandell M (2010) Astronomy and Experimentation. Techné 14:252-269

Schnittman JD (2013) Astrophysics of super-massive black hole mergers. Classical and Quantum Gravity 30(24):244007, 1307.3542

Schödel R, Ott T, Genzel R, Hofmann R, Lehnert M, Eckart A, Mouawad N, Alexander T, Reid MJ, Lenzen R, Hartung M, Lacombe F, Rouan D, Gendron E, Rousset G, Lagrange AM, Brandner W, Ageorges N, Lidman C, Moorwood AFM, Spyromilio J, Hubin N, Menten KM (2002) A star in a 15.2-year orbit around the supermassive black hole at the centre of the Milky Way. Nature419:694-696

Schödel R, Eckart A, Iserlohe C, Genzel R, Ott T (2005) A Black Hole in the Galactic Center Complex IRS 13E? ApJL625:L111-L114, astro-ph/0504474

Schödel R, Eckart A, Alexander T, Merritt D, Genzel R, Sternberg A, Meyer L, Kul F, Moultaka J, Ott T, Straubmeier C (2007) The structure of the nuclear stellar cluster of the Milky Way. A\&A469:125-146, astro-ph/0703178

Schödel R, Amaro-Seoane P, Gallego-Cano E (2017) The stellar cusp around the Milky Way's central black hole. ArXiv e-prints 1702.00219

Schwarzschild K (1916) Über das Gravitationsfeld eines Massenpunktes nach der Einstein'schen Theorie (On the Gravitational Field of a Mass Point According to Einstein's Theory). Abh Königl Preuss Akad Wissenschaften, math-phys Klasse, 424; see arXiv:physics19912033 [physicshist-ph] for an English translation 1916

Sellars W (1963) Science, perception and reality. Atascadero CA: Ridgeview Publishing Companyi $(1963 / 1991)$

Sesana A, Gair J, Berti E, Volonteri M (2011) Reconstructing the massive black hole cosmic history through gravitational waves. Phys. Rev. D83(4):044036, 1011.5893

Shahzamanian B, Eckart A, Valencia-S M, Witzel G, Zamaninasab M, Sabha N, García-Marín M, Karas V, Karssen GD, Borkar A, Dovčiak M, Kunneriath D, Bursa M, Buchholz R, Moultaka J, Straubmeier C (2015a) Polarized light from Sagittarius $A^{*}$ in the near-infrared $\mathrm{K}_{s}$-band. A\&A576:A20

Shahzamanian B, Eckart A, Valencia-S M, Witzel G, Zamaninasab M, Sabha N, García-Marín M, Karas V, Karssen GD, Borkar A, Dovčiak M, Kunneriath D, Bursa M, Buchholz R, Moultaka J, Straubmeier C (2015b) Polarized light from Sagittarius A* in the near-infrared $\mathrm{K}_{s}$-band. A\&A576:A20

Shapiro S, Teukolsky S (1983) Black Holes, White Dwarfs, and Neutron Stars: The Physics of Compact Objects, John Wiley \& Sons Inc; ISBN 0-471-87317-9

Shcherbakov RV, Penna RF, McKinney JC (2012) Sagittarius A* Accretion Flow and Black Hole Parameters from General Relativistic Dynamical and Polarized Radiative Modeling. ApJ755:133, 1007.4832

Shen Y, Greene JE, Ho LC, Brandt WN, Denney KD, Horne K, Jiang L, Kochanek CS, McGreer ID, Merloni A, Peterson BM, Petitjean P, Schneider DP, Schulze A, Strauss MA, Tao C, Trump JR, Pan K, Bizyaev D (2015) The Sloan Digital Sky Survey Reverberation Mapping Project: No Evidence for Evolution in the M - $\sigma$ Relation to z 1. ApJ805:96, 1502.01034

Shen ZQ, Lo KY, Liang MC, Ho PTP, Zhao JH (2005) A size of $\sim 1$ AU for the radio source Sgr $A^{*}$ at the centre of the Milky Way. Nature438:62-64

Slaný P, Kovář J, Stuchlík Z, Karas V (2013) Charged Tori in Spherical Gravitational and Dipolar Magnetic Fields. ApJS205:3, 1302.2356

Smeenk C (2005) False vacuum: Early universe cosmology and the development of inflation. The Universe of General Relativity, edited by J Eisenstaedt and A J Kox, Einstein Studies Vol 11, Boston: Birkhauser (2005)

Spitler LG, Lee KJ, Eatough RP, Kramer M, Karuppusamy R, Bassa CG, Cognard I, Desvignes G, Lyne AG, Stappers BW, Bower GC, Cordes JM, Champion DJ, Falcke H (2014) Pulse Broadening Measurements from the Galactic Center Pulsar J1745-2900. ApJL780:L3, 1309.4673

Su M, Slatyer TR, Finkbeiner DP (2010) Giant Gamma-ray Bubbles from Fermi-LAT: Active Galactic Nucleus Activity or Bipolar Galactic Wind? ApJ724:1044-1082, 1005.5480

Sunyaev R, Churazov E (1998) Equivalent width, shape and proper motion of the iron fluorescent line emission from molecular clouds as an indicator of the illuminating source X-ray flux history. MNRAS297:1279-1291, astro-ph/9805038

Synge JL (1966) The escape of photons from gravitationally intense stars. MNRAS131:463 
Terrier R, Ponti G, Bélanger G, Decourchelle A, Tatischeff V, Goldwurm A, Trap G, Morris MR, Warwick R (2010) Fading Hard X-ray Emission from the Galactic Center Molecular Cloud Sgr B2. ApJ719:143-150, 1005.4807

The eLISA Consortium, Seoane PAea (2013) The Gravitational Universe. ArXiv e-prints 1305.5720

The LIGO Scientific Collaboration, The Virgo Collaboration (2016) Properties of the binary black hole merger GW150914. ArXiv e-prints, 1602038401602.03840

Torres DF, Capozziello S, Lambiase G (2000) Supermassive boson stars: prospects for observational detection. ArXiv:gr-qc/0012031 gr-qc/0012031

Trippe S, Paumard T, Ott T, Gillessen S, Eisenhauer F, Martins F, Genzel R (2007) A polarized infrared flare from Sagittarius A* and the signatures of orbiting plasma hotspots. MNRAS375:764772 , astro-ph/0611737

Tsupko OY, Bisnovatyi-Kogan GS (2013) Gravitational lensing in plasma: Relativistic images at homogeneous plasma. Phys. Rev. D87(12):124009

van Fraassen C (1985) Images of Science: Essays on Realism and Empiricism; Paul M. Churchland (editor); University Of Chicago Press (15. Oktober 1985); ISBN-13: 978-0226106540

Vanhollebeke E, Groenewegen MAT, Girardi L (2009) Stellar populations in the Galactic bulge. Modelling the Galactic bulge with TRILEGAL. A\&A498:95-107

Vasudevan RV, Fabian AC (2007) Piecing together the X-ray background: bolometric corrections for active galactic nuclei, MNRAS 381, 1235. MNRAS381:1235-1251

Verozub L (2006) Sgr A* as probe of the theory of supermassive compact objects without event horizon. Astronomische Nachrichten 327:355

Vincent FH, Paumard T, Perrin G, Mugnier L, Eisenhauer F, Gillessen S (2011a) Astrometric Study of the Complex Environment of Sgr A* in Imaging Mode with the VLTI/GRAVITY Instrument. In: Morris MR, Wang QD, Yuan F (eds) The Galactic Center: a Window to the Nuclear Environment of Disk Galaxies, Astronomical Society of the Pacific Conference Series, vol 439, p 275

Vincent FH, Paumard T, Perrin G, Mugnier L, Eisenhauer F, Gillessen S (2011b) Performance of astrometric detection of a hotspot orbiting on the innermost stable circular orbit of the Galactic Centre black hole. MNRAS412:2653-2664, 1011.5439

Vincent FH, Paumard T, Perrin G, Varniere P, Casse F, Eisenhauer F, Gillessen S, Armitage PJ (2014) Distinguishing an ejected blob from alternative flare models at the Galactic Centre with GRAVITY. MNRAS441:3477-3487, 1404.6149

Vincent FH, Meliani Z, Grandclement P, Gourgoulhon E, Straub O (2015) Imaging a boson star at the Galactic center. ArXiv e-prints, 151004170 1510.04170

Viollier RD, Leimgruber FR, Trautmann D (1992) Halos of heavy neutrinos around baryonic stars. Physics Letters B 297:132-137

Wald R (1984) General Relativity; The University of Chicago Press, Chicago and London; ISBN: 9780226870335

Walker SA, Fabian AC, Russell HR, Sanders JS (2014) The effect of the quasar H1821+643 on the surrounding intracluster medium: revealing the underlying cooling flow. MNRAS442:2809-2816, 1405.7522

Walsh JL, Barth AJ, Ho LC, Sarzi M (2013) The M87 Black Hole Mass from Gas-dynamical Models of Space Telescope Imaging Spectrograph Observations. ApJ770:86, 1304.7273

Wang X, Loeb A (2014) Detecting floating black holes as they traverse the gas disc of the Milky Way. MNRAS441:809-812

Wharton RS, Chatterjee S, Cordes JM, Deneva JS, Lazio TJW (2012) Multiwavelength Constraints on Pulsar Populations in the Galactic Center. ApJ753:108, 1111.4216

Will CM (1974) Perturbation of a Slowly Rotating Black Hole by a Stationary Axisymmetric Ring of Matter. I. Equilibrium Configurations. ApJ191:521-532

Witzel G, Eckart A, Bremer M, Zamaninasab M, Shahzamanian B, Valencia-S M, Schödel R, Karas V, Lenzen R, Marchili N, Sabha N, Garcia-Marin M, Buchholz RM, Kunneriath D, Straubmeier C (2012a) Source-intrinsic Near-infrared Properties of Sgr A*: Total Intensity Measurements. ApJS203:18

Witzel G, Eckart A, Bremer M, Zamaninasab M, Shahzamanian B, Valencia-S M, Schödel R, Karas V, Lenzen R, Marchili N, Sabha N, Garcia-Marin M, Buchholz RM, Kunneriath D, Straubmeier C (2012b) Source-intrinsic Near-infrared Properties of Sgr A*: Total Intensity Measurements. ApJS203:18, 1208.5836

Wollman ER, Geballe TR, Lacy JH, Townes CH, Rank DM (1977) NE II 12.8 micron emission from the galactic center. II. ApJL218:L103-L107

Wrobel JM, Miller-Jones JCA, Middleton MJ (2016) A Very Large Array Search for Intermediatemass Black Holes in Globular Clusters in M81. Astronomical Journal 152:22

Wu Q, Czerny B, Grzedzielski M, Janiuk A, Gu WM, Dong Aj, Cao XF, You B, Yan Z, Sun MY (2016) The Universal Heartbeat; Oscillations in Black Hole Systems Across the Mass-scale. ApJ833:79

Wyithe JSB, Loeb A (2003) Low-Frequency Gravitational Waves from Massive Black Hole Binaries: Predictions for LISA and Pulsar Timing Arrays. ApJ590:691-706, astro-ph/0211556 
Yagi K, Stein LC (2016) Black hole based tests of general relativity. Classical and Quantum Gravity 33(5):054001, 1602.02413

Yuan F, Narayan R (2014) Hot Accretion Flows Around Black Holes. ARA\&A52:529-588, 1401.0586

Yuan F, Quataert E, Narayan R (2003) Nonthermal Electrons in Radiatively Inefficient Accretion Flow Models of Sagittarius A*. ApJ598:301-312, astro-ph/0304125

Yuan F, Bu D, Wu M (2012) Numerical Simulation of Hot Accretion Flows. II. Nature, Origin, and Properties of Outflows and their Possible Observational Applications. ApJ761:130, 1206.4173

Yunes N, Siemens X (2013) Gravitational-Wave Tests of General Relativity with Ground-Based Detectors and Pulsar-Timing Arrays. Living Reviews in Relativity 16:9

Yusef-Zadeh F, Wardle M, Miller-Jones JCA, Roberts DA, Grosso N, Porquet D (2011) Rapid Intrinsic Variability of SGR A* at Radio Wavelengths. ApJ729:44

Yusef-Zadeh F, Arendt R, Bushouse H, Cotton W, Haggard D, Pound MW, Roberts DA, Royster M, Wardle M (2012a) A 3 pc Scale Jet-driven Outflow from Sgr A*. ApJL758:L11

Yusef-Zadeh F, Wardle M, Dodds-Eden K, Heinke CO, Gillessen S, Genzel R, Bushouse H, Grosso N, Porquet D (2012b) An Inverse Compton Scattering Origin of X-Ray Flares from Sgr A*. AJ144:1

Yusef-Zadeh F, Wardle M, Cotton W, Schödel R, Royster MJ, Roberts DA, Kunneriath D (2017) Tidal Distortion of the Envelope of an AGB Star IRS 3 near Sgr A*. ArXiv e-prints

Zajacek M, Karas V, Kunneriath D (2015) Galactic Center Minispiral: Interaction Modes of Neutron Stars. Acta Polytechnica 55:203-214

Zakharov AF (2014) Constraints on a charge in the Reissner-Nordström metric for the black hole at the Galactic Center. Phys. Rev. D90(6):062007

Zamaninasab M, Eckart A, Witzel G, Dovciak M, Karas V, Schödel R, Gießübel R, Bremer M, García-Marín M, Kunneriath D, Mužić K, Nishiyama S, Sabha N, Straubmeier C, Zensus A (2010) Near infrared flares of Sagittarius A*. Importance of near infrared polarimetry. A\&A510:A3

Zeh HD (2007) The physical basis of the direction of time. Fifth edition, ISBN 978-3-540-68000-0, Springer Berlin Heidelberg New York

Zel'dovich YB, Novikov ID (1964) The Radiation of Gravity Waves by Bodies Moving in the Field of a Collapsing Star. Soviet Physics Doklady 9:246

Zubovas K, Nayakshin S, Markoff S (2012) Sgr A* flares: tidal disruption of asteroids and planets? MNRAS421:1315-1324, 1110.6872 


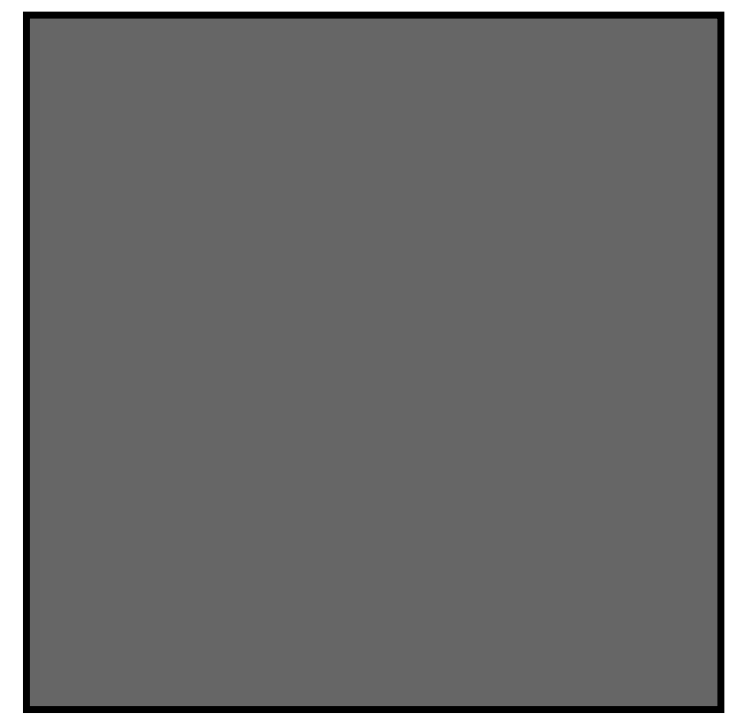

